\title{
Cross-Layer Aided Energy-Efficient Routing Design for Ad Hoc Networks
}

\author{
Jing Zuo, Chen Dong, Soon Xin Ng, Lie-Liang Yang, and Lajos Hanzo
}

\begin{abstract}
4 Abstract-In this treatise, we first review some basic routing 5 protocols conceived for ad hoc networks, followed by some design 6 examples of cross-layer operation aided routing protocols. Specif7 ically, cross-layer operation across the PHYsical layer (PHY), 8 the Data Link layer (DL) and even the NETwork layer (NET)

9 is exemplified for improving the energy efficiency of the entire 10 system. Moreover, the philosophy of Opportunistic Routing (OR) 11 is reviewed for the sake of further reducing the system's energy 12 dissipation with the aid of optimized Power Allocation (PA). The 13 system's end-to-end throughput is also considered in the context 14 of a design example.
\end{abstract}

15 Index Terms-Opportunistic routing, cross-layer, objective func16 tion, near-capacity coding, energy consumption, power allocation.

\section{INTRODUCTION}

18 INCE the commencement of the Defense Advanced Re19 search Projects Agency (DARPA) project [1] developed 20 by the American Defense Department in the 1970s, ad hoc 21 networks have been widely applied in scenarios, including 22 military applications, crisis response, medical care, conference 23 meetings and space exploration. During the past few decades, 24 ad hoc networks attracted substantial research attention as a 25 benefit of their prompt set-up and their ability to self-organize 26 their noncentrally-controlled dynamic topology. Each node of 27 an ad hoc network plays the dual role of being both a terminal 28 and a router under the assumption that not all nodes can directly 29 communicate with each other [2]. Fig. 1(a) and (b) show the 30 difference between the classic infrastructure based network and 31 ad hoc network. Fig. 1(a) shows that the nodes $A, B$ and $C$ 32 communicate with each other under the control of Base Station 33 (BS) 1 and that $A$ communicates with $E$ via BS 1 and BS 2. 34 However, Fig. 1(b) shows that $A$ can only communicate with $E$ 35 by relying on $B, C$ and $D$ as its Relay Nodes (RNs). Each node 36 has to discover its own neighbor list.

37 The characteristics of ad hoc networks impose a number of 38 open problems, which constitute challenges for the protocol 39 design. For example, the scalability, the energy-efficiency, the

Manuscript received January 9, 2014; revised July 6, 2014 and October 31, 2014; accepted January 17, 2015. The research leading to these results has received funding from the European Union's Seventh Framework Programme (FP7/2012-2014) under grant agreement no 288502. The financial support of the China-UK Scholarship Council, and of the RC-UK under the auspices of the IU-ATC initiative is also gratefully acknowledged.

The authors are with the School of Electronics and Computer Science (ECS), University of Southampton, Southampton SO17 1BJ, U.K. (e-mail: jz08r@ecs. soton.ac.uk; cd2g09@ecs.soton.ac.uk; sxn@ecs.soton.ac.uk; 1ly@ecs.soton. ac.uk; 1h@ecs.soton.ac.uk; http://www-mobile.ecs.soton.ac.uk).

Color versions of one or more of the figures in this paper are available online at http://ieeexplore.ieee.org.

Digital Object Identifier 10.1109/COMST.2015.2395378

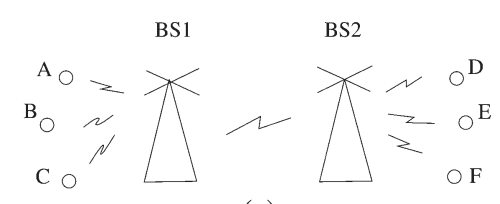

(a)

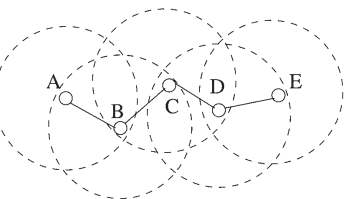

(b)

Fig. 1. Categories of wireless networks. (a) The infrastructure network; (b) Ad hoc network.

Quality of Service (QoS) and the security are challenging 40 problems to be solved and to be further improved. Hence the 41 emphasis of this treatise is on the design of routing protocols 42 relying on cross-layer interaction for improving the attainable 43 system performance, such as the Normalized Energy Consump- 44 tion (NEC) and the end-to-end throughput.

A. Cross-Layer Design

The International Standards Organization (ISO) created the 47 SubCommittee 16 (SC16) in 1977 for developing an architec- 48 ture, which could serve as a framework for the definition of 49 standard protocols. At the end of 1979, the Reference Model 50 of Open System Interconnection (OSI) was adopted by the 51 parent of SC16, namely, Technical Committee (TC97). The 52 OSI Reference Model was also recognized by the International 53 Telegraph and Telephone Consultative Committee (CCITT) 54 Rappporteur's Group on Public Data Network Services. The 55 OSI Reference Model consists of seven layers, which are the 56 PHYsical layer (PHY), the Data Link layer (DL), the NET- 57 work layer (NET), the transport layer, the session layer, the 58 presentation layer and the application layer. The benefit of 59 this layering technique is to group the similar communication 60 functions into these logical layers. A layer has to cooperate with 61 the layer above it and the layer below it. However, when the 62 Transmission Control Protocol (TCP) of the transport layer and 63 the Internet Protocol (IP) of the network layer were defined, 64 the five-layer model (TCP/IP model) became the dominant one. 65 More explicitly, the TCP/IP model consists of the application 66 layer, the transport layer, the NETwork (NET) layer, the Data 67 Link (DL) layer and the PHYsical (PHY) layer [3]-[6]. Fig. 268 illustrates the structure of the TCP/IP model and the main 69 functions of each layer.

The functions of these layers are briefly highlighted below: 71

- The physical layer: The PHY layer concentrates on both 72 the physical devices and on the transmission media. Pro- 73 viding a diversity and/or multiplexing gain with the aid 74 of multiple antennas is capable of improving the integrity 75 and/or throughput of data transmission. Additionally, the 76 


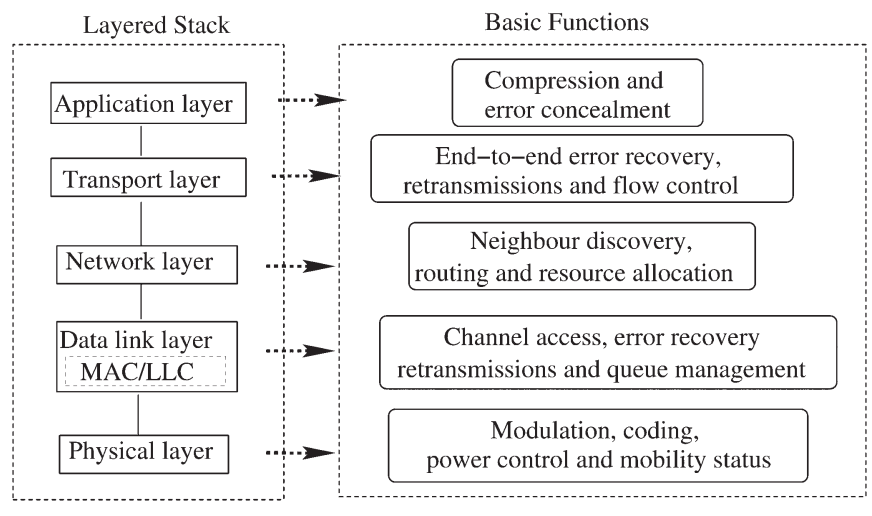

Fig. 2. Layered stack and the main functions in each layer. adjustment of the transmit power, the design of the coding and modulation schemes as well as the effects of mobility and/or propagation effects constitute important design factors of the physical layer.

- The data link layer: The DL layer of Fig. 2 is concerned with the media access, the error recovery, the retransmission and the queue management functions. It consists of two sub-layers, namely the Media Access Control (MAC) sublayer and the Logical Link Control (LLC) sublayer [7].

- The network layer: The NET layer is responsible for the neighbor discovery, routing and resource allocation functions. Routing is the main function of the network layer, guiding a packet through the network from a source to the destination [8], [9]. Numerous routing protocols have been designed based on the IP protocol for satisfying the requirements of wireless ad hoc networks, which fundamentally predetermines the attainable performance in terms of the Packet Loss Ratio PLR, the end-to-end delay and the network's throughput.

- The transport layer: The transport layer is responsible for flow control, congestion control, error recovery, packet reordering and for the end-to-end connection setup. It assists the application layer of Fig. 2 in allocating/mapping the flows to different routes, which are found in the NET layer. It also assists by monitoring the end-to-end data transmission and in avoiding network congestion [10], [11].

- The application layer: The application layer constitutes the interface to the end user in the TCP/IP model of Fig. 2. By considering the requirements of the end user, it divides the user services into different categories, such as for example, real-time and non-real-time services, continuous and intermittent services, Constant Bit Rate (CBR) and Variable Bit Rate (VBR) multimedia services, etc. [12].

Again, although the layered architecture has its own ad111 vantages and performs well in wired networks in terms of 112 portability, flexibility and low design complexity, it is not 113 suitable for wireless networks, especially in wireless ad hoc 114 networks. The reason for its inadequacy in wireless scenarios 115 is that the services offered by the layers to those above them 116 in Fig. 2 are realized by specifically tailored protocols for the 117 different layers and that the architecture forbids direct com118 munication between non-adjacent layers. The communication 119 between adjacent layers is limited to procedure calls and to their

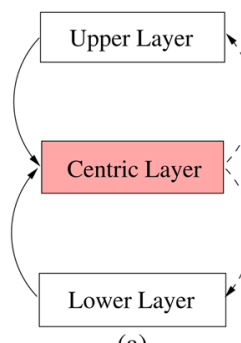

(a)

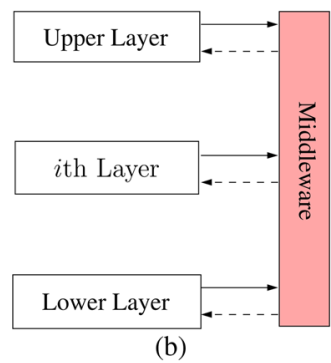

Fig. 3. Conceptual illustration of cross-layer design methods. (a) Layercentric solution; (b) centralized solution.

responses. Moreover, the hostile wireless links impose several 120 new problems on the associated protocol design that cannot 121 be readily handled by the layered architecture [13]. More 122 explicitly, having a strict layered design is not flexible enough to 123 cope with the dynamics of Mobile Ad hoc NETwork (MANET) 124 environments and will thus result in a low performance [14]. 125 Thus, the mutual impact of the layers on each other cannot 126 be ignored [15]. Hence the concept of cross-layer design has 127 been proposed in an attempt to achieve a performance gain by 128 exploiting the close interaction amongst the different layers. 129 Srivastava and Motani [13] defined "Cross-Layer operation" 130 as: "Protocol design by the violation of a reference layered 131 communication architecture is cross-layer design with respect 132 to the particular layered architecture", while Jurdak [15] define 133 it as: "Cross-layer design with respect to a reference layered 134 architecture is the design of algorithms, protocols, or architec- 135 tures that exploit or provide a set of inter-layer interactions that 136 is a superset of the standard interfaces provided by the reference 137 layered architecture". Therefore, cross-layer operation may be 138 interpreted as the 'violation' of the layered architecture seen 139 in Fig. 2, which requires more interaction amongst the layers, 140 beyond the interaction between the adjacent layers. Cross-layer 141 design clearly requires information exchange between layers, as 142 well as adaptivity to this information at each layer and a certain 143 grade of diversity built into each layer for the sake of improving 144 the achievable robustness [5].

There are two basic methods of information sharing in cross- 146 layer design [16]. One of them makes the variables of a specific 147 layer visible to the other layers, which is referred to as a layer- 148 centric solution. The other relies on a shared middleware [15], 149 [16], which provides the service of storage/retrieval of infor- 150 mation to all layers, which is termed as a centralized solution. 151 Fig. 3 illustrates how these two cross-layer solutions operate. 152

The basic principles of the above-mentioned pair of cross- 153 layer solutions are:

- The layer-centric solution: A certain layer is allowed to 155 be the central layer, which controls the cross-layer adap- 156 tation by accessing the internal protocol parameters and 157 algorithms of the other layers, as shown in Fig. 3(a). Al- 158 though this approach significantly improves the attainable 159 system performance, it violates the layered architecture, 160 since it requires access to the internal variables of other 161 layers.

- The centralized solution: A middleware or a system-level 163 monitor (centralized optimizer) is employed for estimating 164 both the availability of resources and the environmental 165 
TABLE I

Major Contributions of Cross-Layer Design in Ad Hoc Networks

\begin{tabular}{|l|l|l|}
\hline Year & Authors & Contribution \\
\hline \hline 2005 & Setton et al. [18] & $\begin{array}{l}\text { Explored the potential synergies of exchanging information between different layers to support real-time } \\
\text { video streaming. }\end{array}$ \\
\hline 2006 & Liu et al. [19] & $\begin{array}{l}\text { Proposed a scheduling algorithm at the MAC layer for multiple connections under diverse QoS } \\
\text { requirements, where each connection employs both adaptive modulation and coding at the PHY layer } \\
\text { for transmission over wireless channels. }\end{array}$ \\
\hline 2007 & $\begin{array}{l}\text { Huang } \\
\text { and Letaief [20] }\end{array}$ & $\begin{array}{l}\text { Proposed a cross-layer optimization framework to jointly design the scheduling, power control and } \\
\text { adaptive modulation. }\end{array}$ \\
\hline 2008 & $\begin{array}{l}\text { Zhang } \\
\text { and Zhang [21] }\end{array}$ & $\begin{array}{l}\text { Reviewed the state-of-the-art on the cross-layer paradigm and discussed the open issues related to cross- } \\
\text { layer design for QoS support. }\end{array}$ \\
\hline 2009 & $\begin{array}{l}\text { Oh } \\
\text { and Chen [22] }\end{array}$ & $\begin{array}{l}\text { Presented a cross-layer design for reliable video transmission based on a multichannel MAC protocol in } \\
\text { the context of time division multiple access. }\end{array}$ \\
\hline 2010 & $\begin{array}{l}\text { Chu } \\
\text { and Wang [23] }\end{array}$ & $\begin{array}{l}\text { Presented cross-layer centralized and distributed scheduling algorithms, which exploited the PHY layer } \\
\text { channel information to opportunistically schedule cooperative spatial multiplexed transmissions between } \\
\text { MIMO-based nodes. }\end{array}$ \\
\hline 2011 & $\begin{array}{l}\text { Ghosh } \\
\text { and Hamouda [24] }\end{array}$ & $\begin{array}{l}\text { Proposed a cross-layer antenna selection algorithm for improving the transmission efficiency in cognitive } \\
\text { MIMO-aided ad hoc networks. }\end{array}$ \\
\hline 2012 & Mardani et al. [25] & $\begin{array}{l}\text { Jointly considered flow control, multipath routing and random access control based on network utility } \\
\text { maximization. }\end{array}$ \\
\hline 2013 & Uddin et al. [26] & $\begin{array}{l}\text { Studied cross-layer design in random-access-based fixed wireless multihop networks under a physical } \\
\text { interference model. }\end{array}$ \\
\hline 2014 & $\begin{array}{l}\text { Tang } \text { et al. [27] } \\
\text { Proposed a cross-layer distributed approach for maximizing the network throughput by jointly selecting } \\
\text { stable routes and assigning channels based on mobility prediction. }\end{array}$ \\
\hline \hline
\end{tabular}

178 The cross-layer operation aided design of wireless ad hoc 179 networks poses challenges mainly due to the time-variant char180 acteristics of wireless channels. The signal is substantially more 181 vulnerable to the effects of noise, fading and interference than 182 in benign fixed networks, leading to potential performance 183 degradations within the higher layers. For example, a packet has 184 to be retransmitted in the DL layer or the transmit power has to 185 be adjusted to guarantee its high-integrity transmission, which 186 may impose interference on other nodes or promote aggressive 187 contention for channel access. In the NET layer, the current 188 route may become invalid and hence route maintenance/repair 189 has to be activated or even a new route discovery process has to 190 be initiated. As a result, potentially more energy is consumed 191 and the end-to-end delay is increased, while the end-to-end 192 throughput is reduced. Therefore, careful adaptation of the 193 protocol stack should be used at each layer to compensate for 194 the variations at that layer, depending on the specific time scale 195 of these variations [5]. Both the local adaptation of parameters 196 within each layer and the adaptation based on the other layers 197 have to be considered. For example, the transmit power, the 198 signal processing hardware's power dissipation, the information 199 transmit rate, the coding and modulation schemes, the Frame 200 Error Ratio (FER) and the mobility in the PHY layer consti- tute important parameters, which may be beneficially shared 201 with other layers. The protocol design of the upper layers has 202 to consider the information gleaned from the PHY layer for 203 minimizing the energy consumption, the resource allocation, 204 scheduling and the queueing management, while maintaining 205 a certain QoS guarantee. Meanwhile, the number of retransmis- 206 sions, as well as both the routing and network topology related 207 information received from the upper layers may be beneficially 208 shared. Additionally, node cooperation also calls for cross-layer 209 design [13]. Table I is presented for discussing the previous 210 work on cross-layer design in a compact manner.

These cross-layer aided designs may be classified into several 212 categories according to their different application requirements. 213 They might be designed for reducing the energy consumption, 214 the end-to-end delay [21], for improving the network's through- 215 put [18], [22], [24], [26], [27], for striking a flexible tradeoff 216 between any two of them [20], [23], and even for multiple- 217 constraint optimization [19], [21].

As detailed above, cross-layer design has substantial benefits, 219 but it has its own disadvantages as well. For example, the cross- 220 layer interactions create dependencies amongst the layers, 221 which will affect not only the layer concerned, but also the other 222 layers. Hence, a complete redesign of the operational networks 223 and protocols will lead to a high implementational cost [16]. 224 Therefore, cross-layer design should be carefully crafted, be- 225 cause once the seven-layer OSI structure is violated, the benefits 226 of independent, layer-specific protocol design will disappear 227 [13], [28]. The effects of any protocol chosen in every single 228 layer on the overall system has to be carefully considered.

\section{B. Categories of Ad Hoc Routing Protocols}

The NET layer of Fig. 2 plays a key role in ad hoc networks, 231 which substantially influences the performance of the overall 232 system. The NET layer is responsible both for allocating IP 233 


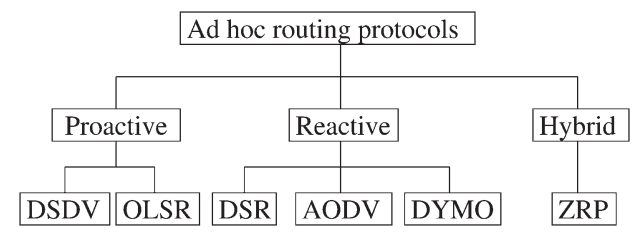

Fig. 4. Categorization of ad hoc routing protocols (DSDV: DestinationSequenced Distance Vector routing; OLSR: Optimized Link State Routing; DSR: Dynamic Source Routing; AODV: Ad-hoc On-demand Distance Vector routing; DYMO: DYnamic Manet On-demand routing; ZRP: Zone Routing Protocol).

234 addresses and for choosing the right route for communication 235 between the source and destination. The routing protocols of $\mathrm{ad}$ 236 hoc networks may be classified as proactive routing, reactive 237 routing and hybrid routing [29], as shown in Fig. 4.

238 The proactive routing periodically transmits "hello" packets 239 for the sake of identifying all possible routes in the network. 240 Hence every node has to maintain a routing table, which stores 241 the route spanning from itself to all other available nodes. The 242 advantage of this kind of routing protocol is that the route 243 discovery time is low. By contrast, its disadvantage is that each 244 node has to maintain a routing table. If the number of nodes in 245 the network becomes high, then the routing table becomes large 246 and hence requires a large memory. On the other hand, periodi247 cally sending "hello" packets increases the network control load 248 imposed. The so-called Destination-Sequenced Distance Vector 249 (DSDV) [30] protocol and the Optimized Link State Routing 250 (OLSR) [31] protocol are typical proactive routing protocols, 251 as seen in Fig. 4.

252 The reactive routing protocols are source-driven, implying 253 that they transmit route discovery packets to find a route to 254 the destination, when there is sufficient data scheduled for 255 transmission in the buffer, instead of periodically broadcasting 256 the "hello" packets. As a benefit, not all nodes have to maintain 257 a route table for storing the routes leading to all other nodes. In258 stead, they only store routes that were found during the process 259 of route discovery. This technique reduces the network control 260 load compared to the proactive routing protocols of Fig. 4. The 261 disadvantage of this routing protocol family is however that 262 their delay is increased, because a route has to be found to the 263 destination, when no routes leading to the destination exist in 264 the route table. Additionally, the nodes' movement changes the 265 network's topology, which hence requires the transmission of 266 more control packets for the sake of maintaining the current 267 communication session. As seen in Fig. 4, the Dynamic Source 268 Routing (DSR) [32], Ad-hoc On-demand Distance Vector 269 (AODV) [33] and DYnamic Manet On-demand (DYMO) [34] 270 routing protocols constitute typical reactive routing protocols.

271 Based on beneficially combining the advantages, whilst 272 avoiding the disadvantages of the above-mentioned protocol 273 families, hybrid routing protocols may also be conceived. We 274 may divide the entire ad hoc network into several small areas 275 and in each area proactive routing may be employed for estab276 lishing a link for all nodes. By contrast, between the areas, reac277 tive routing protocols may be adopted for reducing the number 278 of control packets required. Hybrid protocols are widely applied 279 in large ad hoc networks. The so-called Zone Routing Protocol 280 (ZRP) [35] is a typical hybrid routing protocol.

\section{Review of Cross-Layer Aided Routing Protocols}

This treatise is mainly dedicated to cross-layer operation 282 aided routing design in ad hoc networks, hence we list the 283 major contributions to the literature of cross-layer aided routing 284 protocols conceived for ad hoc networks in Table II.

285

Similarly, these cross-layer aided routing protocols may be 286 classified into several categories according to their different 287 application requirements. They might be designed for reducing 288 the energy consumption [38], [48], [52], the end-to-end delay 289 [47], for improving the network's throughput [39], [49], [54], 290 for striking a flexible tradeoff between any two of them [26], 291 [42], [45], [46], [50], [51], and even for multiple-constraint 292 optimization [36], [41], [43], [44].

\section{Review of Energy-Efficient Routing Protocols}

As mentioned in Section I-C, cross-layer design may be 295 studied based on diverse application requirements. This paper 296 focuses on cross-layer design techniques conceived for reduc- 297 ing the energy consumption. since energy saving in wireless 298 ad hoc networks is of salient importance in the interest of 299 mitigating the problem of limited battery supply at each 300 node. In ad hoc networks the nodes actively and voluntarily 301 participate in constructing a network and act as relays for 302 other nodes. As a result of node-mobility, the Channel State 303 Information (CSI) varies and hence a substantial amount 304 of control messages have to be exchanged across the net- 305 work to maintain reliable communications between certain 306 pairs of nodes, which potentially imposes a high energy- 307 consumption. Therefore, minimizing the energy consump- 308 tion becomes extremely important. Numerous power-aware 309 routing protocols were proposed in [55] for improving the 310 energy efficiency from a multiuser networking perspective. 311 Firstly, a compact-form review of energy-efficient single-layer 312 routing design is provided in Table III.

Moreover, cross-layer optimized power control has been 314 widely exploited [66]-[71] for maintaining the required target- 315 integrity at a low power in realistic propagation environments. 316 A physical-layer-oriented routing protocol supported by sophis- 317 ticated power control was proposed in [66] for a Line-Of-Sight 318 (LOS) and shadow faded scenario, where the estimated end-to- 319 end BER of a multi-hop path was used as the route selection 320 metric. Furthermore, an adaptive relaying strategy switching 321 between the Amplify-and-Forward (AF) and the Decode-and- 322 Forward (DF) schemes was proposed in [67] for reducing both 323 the energy consumption as well as the delay of the system. As 324 a further design dilemma, the influence of the 'small number 325 of long hops' versus the 'many short hops' philosophy on the 326 energy consumption was studied in [68]-[70]. It was indicated 327 in [68] that the 'small number of long hops' routing scheme 328 was better than the 'many short hops' routing scheme provided 329 that near-capacity coding strategies combined with a relatively 330 short block length were employed, because a substantial SNR 331 loss was exhibited by the 'many short hops' based routing 332 scheme. Moreover, it was demonstrated in [69] that 'many 333 short hops' perform well in energy-limited scenarios relying on 334 spatial reuse, even in the absence of interference cancellation, 335 while using a 'small number of long hops' is more suitable for 336 
TABLE II

Major Contributions of Cross-Layer Aided Routing Protocols in Ad Hoc Networks

\begin{tabular}{|c|c|c|}
\hline Year & Authors & Contribution \\
\hline 2002 & $\begin{array}{l}\text { Goldsmith } \\
\text { and Wicker [36] }\end{array}$ & $\begin{array}{l}\text { Reviewed each layer's protocol and emphasized the necessity of cross-layer design, particularly in energy- } \\
\text { limited scenarios. }\end{array}$ \\
\hline \multirow[t]{2}{*}{2005} & Souryal et al. [37] & Proposed efficient channel-quality-aware adaptive routing relying on adaptive modulation. \\
\hline & Lee et al. [38] & Combined power-aware routing with a MAC layer algorithm for minimizing the total consumed power. \\
\hline \multirow[t]{4}{*}{2006} & $\begin{array}{l}\text { Johansson } \\
\text { and Xiao [39] }\end{array}$ & $\begin{array}{l}\text { Jointly optimized the end-to-end communication rates, routing, power allocation and transmission } \\
\text { scheduling of a network. }\end{array}$ \\
\hline & Mao et al. [40] & $\begin{array}{l}\text { proposed a Genetic Algorithm (GA)-based application-centric cross-layer approach for minimizing video } \\
\text { distortion. }\end{array}$ \\
\hline & $\begin{array}{l}\text { Abdrabou } \\
\text { and Zhuang [41] }\end{array}$ & Presented a position-aware QoS routing scheme by considering its interactions with the MAC. \\
\hline & Zhang et al. [42] & $\begin{array}{l}\text { Addressed the topic of energy-efficient routing subject to both packet delay and multi-access interference } \\
\text { constraints. }\end{array}$ \\
\hline \multirow[t]{2}{*}{2007} & $\begin{array}{l}\text { Kompella } \\
\text { et al. }[43]\end{array}$ & $\begin{array}{l}\text { Optimized the performance of Multiple Description (MD) video subject to certain routing and link layer } \\
\text { constraints. }\end{array}$ \\
\hline & $\begin{array}{l}\text { Chiang } \\
\text { et al. }[44]\end{array}$ & $\begin{array}{l}\text { Surveyed the functional modules, such as congestion control, routing, scheduling, random access, power } \\
\text { control and channel coding. }\end{array}$ \\
\hline \multirow[t]{2}{*}{2008} & Phan et al. [45] & $\begin{array}{l}\text { Presented a cross-layer optimization approach jointly considering the design of the MAC, routing and } \\
\text { energy distribution. }\end{array}$ \\
\hline & Liu et al. [46] & $\begin{array}{l}\text { Jointly optimized the power and bandwidth allocation at each node and designed multihop/multipath } \\
\text { routing for a MIMO-based wireless } a d \text { hoc network. }\end{array}$ \\
\hline \multirow[t]{2}{*}{2009} & $\begin{array}{l}\text { Abdrabou } \\
\text { and Zhuang [47] }\end{array}$ & $\begin{array}{l}\text { Proposed a routing scheme based on a geographical on-demand routing protocol, which is capable of } \\
\text { guaranteeing a certain maximum end-to-end delay. }\end{array}$ \\
\hline & Li et al. $[48]$ & $\begin{array}{l}\text { Proposed a combined multi-rate power controlled MAC protocol and routing protocol relying on the } \\
\text { effective transport capacity as the routing metric. }\end{array}$ \\
\hline \multirow[t]{2}{*}{2010} & Ding et al. [49] & $\begin{array}{l}\text { Proposed a ROuting and dynamic Spectrum-Allocation (ROSA) algorithm aiming for maximizing the } \\
\text { network's throughput by performing joint routing, dynamic spectrum allocation, scheduling and } \\
\text { transmit power control. }\end{array}$ \\
\hline & Lu et al. [50] & $\begin{array}{l}\text { Presented Joint Channel Assignment and Cross-layer Routing (JCACR) by employing two metrics, namely } \\
\text { the Channel Utilization Percentage (CUP) and the Channel Selection Metric (CSM). }\end{array}$ \\
\hline \multirow[t]{2}{*}{2011} & $\begin{array}{l}\text { Ding } \\
\text { and Leung [51] }\end{array}$ & $\begin{array}{l}\text { Proposed cross-layer routing applying both cooperative transmission and path selection for striking a } \\
\text { tradeoff between the transmit power consumption and the end-to-end reliability. }\end{array}$ \\
\hline & $\begin{array}{l}\text { Tavli and } \\
\text { Heinzelman [52] }\end{array}$ & Presented real-time multicasting based routing. \\
\hline 2012 & Syue et al. [53] & Proposed a relay-aware cooperative routing protocol relying on cross-layer design. \\
\hline 2013 & Pan et al. [54] & $\begin{array}{l}\text { Investigated the path selection problem based on the cross-layer optimization in on flow routing, multihop } \\
\text { Cognitive Radio Networks(CRNs) under constraints link scheduling and CR source's budget. }\end{array}$ \\
\hline 2014 & Uddin et al. [26] & $\begin{array}{l}\text { Studied cross-layer design in random-access-based fixed wireless multihop networks under a physical } \\
\text { interference model. }\end{array}$ \\
\hline
\end{tabular}

TABLE III

Major Contributions of Single Layer Energy-Efficient Routing Protocols in Ad Hoc Networks

\begin{tabular}{|l|l|l|}
\hline Year & Authors & Contribution \\
\hline \hline 2005 & Muruganathan et al. [56] & $\begin{array}{l}\text { Proposed a centralized routing protocol referred to as a base-station controlled dynamic clustering } \\
\text { protocol, which distributes the energy dissipation evenly among all sensor nodes for improving the } \\
\text { network lifetime and for achieving average energy savings. }\end{array}$ \\
\hline 2006 & Zhu et al. [57] & $\begin{array}{l}\text { Proposed a minimum energy dissipation routing protocol based on an accurate model, which took } \\
\text { into account the energy consumption of both the data packets, as well as of the control packets and } \\
\text { retransmissions. }\end{array}$ \\
\hline 2007 & $\begin{array}{l}\text { Baek } \\
\text { and Veciana [58] }\end{array}$ & $\begin{array}{l}\text { Investigated the employment of proactive multipath routing to achieve a tradeoff between the energy } \\
\text { cost of spreading traffic and the improved spatial balance of energy. }\end{array}$ \\
\hline 2008 & Eidenbenz et al. [59] & $\begin{array}{l}\text { Designed an energy-efficient distributed algorithm based on the so-called side payment scheme in } \\
\text { conjunction with a game-theoretic technique to achieve truthfulness for the rational selfish nodes. }\end{array}$ \\
\hline 2009 & Liang et al. [60] & $\begin{array}{l}\text { Designed energy-efficient routing algorithms, which constructed a shared multicast tree spanning all } \\
\text { terminal nodes, while ensuring that the total energy consumption of realizing all-to-all multicasting } \\
\text { was minimized. }\end{array}$ \\
\hline 2011 & $\begin{array}{l}\text { Ma } \text { et al. } \text { [61] } \\
\text { and Yang [62] }\end{array}$ & $\begin{array}{l}\text { Proposed a virtual-link-reduction-based broadcasting protocol using directional antennas. } \\
\text { Proposed an online computable discrete-time mathematical energy model for characterising the } \\
\text { battery discharging behavior and proposed a battery-aware routing schemethat incorporates battery } \\
\text { awareness into routing protocols. }\end{array}$ \\
\hline 2012 & $\begin{array}{l}\text { Akhtar } \text { et al. } \text { [63] } \\
\text { Designed a cooperative routing algorithm, which took the electronic power consumption into } \\
\text { consideration, when constructing the minimum-power route leading from source to destination. }\end{array}$ \\
\hline 2013 & $\begin{array}{l}\text { Lu } \\
\text { and Zhu [64] }\end{array}$ & $\begin{array}{l}\text { Proposed an energy-efficient genetic algorithm aided mechanism, which depended on bounded end- } \\
\text { to-end delay and minimum energy cost of the multicast tree, to solve QoS based multicast routing } \\
\text { problems. }\end{array}$ \\
\hline \hline
\end{tabular}




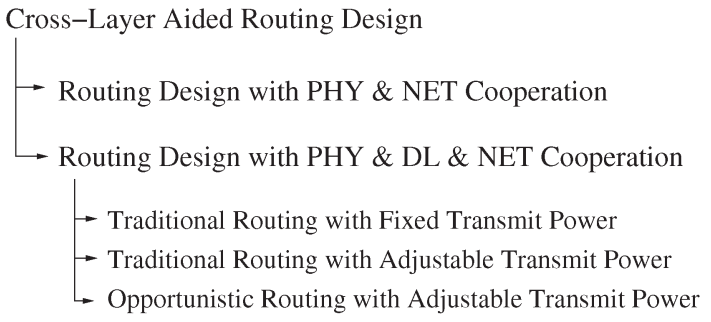

Fig. 5. Structure of this treatise.

337 bandwidth-limited scenarios. Therefore, the routing algorithms 338 should be carefully designed, when jointly considering both 339 the achievable energy-efficiency and the attainable bandwidth340 efficiency. The tradeoffs between energy- and bandwidth341 efficiency were studied in [70], where it was found that at high 342 end-to-end data rates the routes associated with fewer hops 343 minimize the energy consumption, while at lower end-to-end 344 data rates the routes having more hops mitigate it.

\section{E. Outline}

346 Based on the discussions in the previous sections, the rest of 347 the paper is organized as follows: First, we study the cross-layer 348 aided routing design jointly considering both the PHY layer and 349 the NET layer [72], as shown in Section II; Then in Section III 350 we further investigate the cross-layer aided routing design 351 concept by jointly considering the PHY layer, the DL layer 352 and the NET layer [73], [74]. We commence by considering 353 Traditional Routing (TR) relying on a fixed transmit power 354 in Section III-A, while TR combined with Power Allocation 355 (PA) is discussed in Section III-B and Opportunistic Routing 356 (OR) using PA is studied in Section III-C; Finally, Section IV 357 concludes this treatise and offers some design guidelines. Fig. 5 358 lists the structure of this paper.

359 The notations used in this treatise are defined as follows:

$360-N$ : the number of nodes in the network;

361 - $H$ : the number of hops in an established route;

362 - $N_{r}$ : the maximum number of MAC retransmissions, in-

363 cluding the first transmission attempt;

$364-P_{t}$ : the transmit power of each node;

$365-P_{t_{i}}$ : the transmit power in the $i$-th node of the established route;

- FER $R_{i}$ : the FER of the $i$-th link in an established route;

- $p_{i}$ : the successful reception probability of the $i$-th link, where $p_{i}=1-F E R_{i}$;

- $E_{T}$ : the sum of the energy dissipated by all the nodes in the network, including the data packets and the control packets;

- $\bar{E}_{T}$ : the overall energy dissipation $E_{T}$ normalized by the number of bits received in the application layer of the destination;

- $E_{\text {total }}$ : the sum of the energy dissipated by the data packets during their transmission in the network;

- $\bar{E}_{\text {total }}$ : the total energy dissipation $E_{\text {total }}$ normalized by the end-to-end successful reception probability, which is the average energy consumption dissipated by the entire system during the successful delivery of a packet from the source to the destination;

- $R_{e 2 e}$ : the number of information bits successfully delivered to the destination per second.

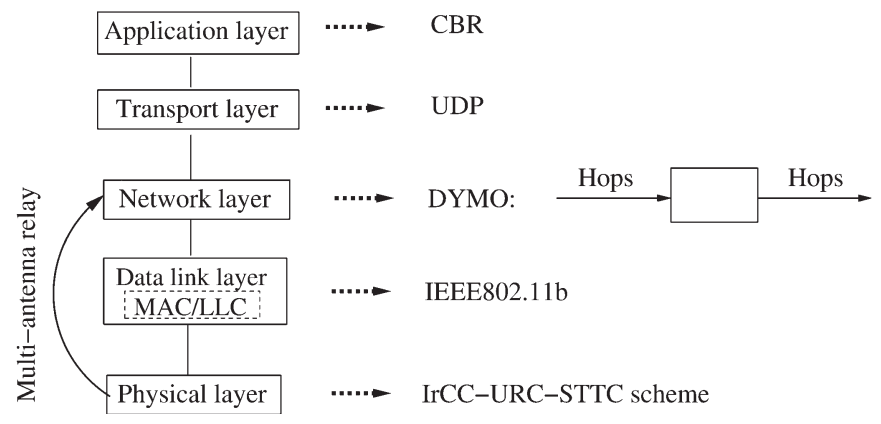

Fig. 6. System model of the energy-efficient routing with PHY \& NET cooperation in ad hoc networks.

\section{Routing Design With PHY \& NET Cooperation 384}

Energy-efficient wireless network design has recently at- 385 tracted wide-spread research attention [75]. Diverse error- 386 resilient Forward Error Correction (FEC) schemes were 387 proposed in [76] for achieving a low Bit Error Ratio (BER) at 388 near-capacity Signal-to-Noise Ratio (SNR) values. Therefore, 389 the effective transmission range can be improved, when the 390 required received signal power is reduced. Again, an Irregular 391 Convolutional Coded, Unity-Rate Coded and Space-Time Trel- 392 lis Coded (IrCC-URC-STTC) scheme has been proposed for 393 cooperative communications in [77]. Several Single-Antenna 394 RNs (SAs) were activated between the source and the destina- 395 tion. The RNs roaming closest to their optimal locations were 396 activated based on a technique relying on EXtrinsic Information 397 Transfer (EXIT) charts [78] in conjunction with near-capacity 398 code design principles, which were detailed for example in [79]. 399

However, the solution disseminated in [72] aims for minimiz- 400 ing the energy consumption by the joint design of both the PHY 401 and NET layers with the assistance of Multiple-Antenna Aided 402 Relay Nodes (MA-RN), as shown in the system model of Fig. 6. 403 Although the routing metric is still the number of hops, the em- 404 ployment of MA-RNs assists in reducing the potential number 405 of hops from the source to the destination, when dissipating a 406 given transmit power at each node. Therefore MA-RNs are ca- 407 pable of reducing the entire system's energy consumption. The 408 influence of the number of MA-RNs in a system will be studied 409 in Section II-C. Both the perfect capacity-achieving coding ab- 410 straction and a realistic near-capacity coding scheme, namely a 411 three-stage-concatenated IrCC-URC-STTC arrangement is em- 412 ployed in the PHY layer. The IEEE802.11b regime [7] is used 413 in the DL layer. In the NET layer, the more efficient DYMO 414 routing protocol [34] is employed, because the DYMO routing 415 protocol imposes a lower network control load and it is more 416 flexible in a high-mobility environment. However, the scenario 417 considered in [72] is a stationary scenario. The investigation of 418 high-mobility scenarios was set aside for its future study. The 419 User Data Protocol UDP [80] is employed in the transport layer 420 and CBR data streaming is used in the application layer. The 421 channel model employed is an Additive White Gaussian Noise 422 (AWGN) channel subjected to both inverse-second-power free- 423 space path loss and to uncorrelated Rayleigh fading.

\section{A. Near-Capacity Coding Schemes}

Each MA-RN is assumed to be equipped with two anten- 426 nas. If more than one MA-RN exist in the multi-hop ad hoc 427 


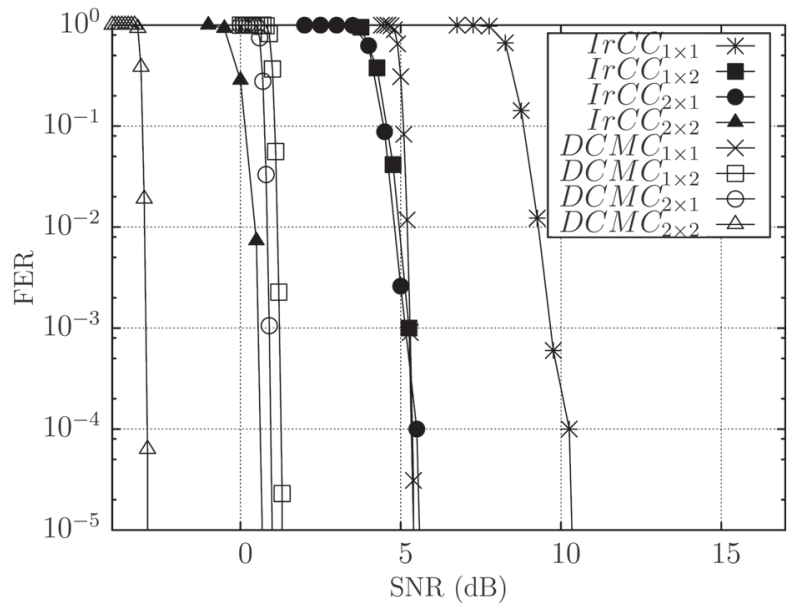

Fig. 7. FER performance of the four types of links, for example at the frame length of 1500 bits, of the uncorrelated Rayleigh fading channel for DCMCcapacity-based scheme and the IrCC-URC-STTC scheme, where $D C M C_{T \times R}$ represents the DCMC-capacity-based scheme, and $\operatorname{IrCC} T \times R$ represents the IrCC-URC-STTC scheme. Additionally, the subscript ' $T \times R$ ' represents having $T$ transmit antennas and $R$ receive antennas. Moreover, the overall FEC code rate is $R_{c}=0.5$, the effective throughput is $1 \mathrm{bps}$ (bits/symbol), the number of transmitted frames is 10000 and the IrCC has 17 component codes, associated with the weights $[0.049,0,0,0,0,0.24,0.16,0.12,0.035,0.102,0,0.071$, $0.093,0,0.091,0,0.039]$

428 network considered, then four different types of links may 429 appear. Specifically, there exists the SA-RN to SA-RN, SA$430 \mathrm{RN}$ to MA-RN, MA-RN to SA-RN and finally the MA-RN to 431 MA-RN links. All the MA-RNs employ the Quadrature Phase432 Shift Keying (QPSK)-assisted IrCC-URC-STTC scheme, while 433 all the SA-RNs employ the 8-ary Phase-Shift Keying (8PSK)434 assisted IrCC-URC scheme.

435 For example, the FER performance of all the four links at the 436 frame length of 1500 bits characterized by the Discrete-input 437 Continuous-output Memoryless Channel's (DCMC)-capacity 438 [81] and that of the IrCC-URC-STTC scheme is portrayed in 439 Fig. 7. It can be observed that the IrCC-URC-STTC scheme 440 performs close to the DCMC-capacity based scheme at a given $441 \mathrm{SNR}$ value. Meanwhile, the $\operatorname{IrCC}_{2 \times 2}$ scheme has a $5 \mathrm{~dB}$ gain 442 compared to $\operatorname{IrCC} C_{2 \times 1}$ or $\operatorname{IrCC} C_{1 \times 2}$ arrangements and has a nearly $44310 \mathrm{~dB}$ gain compared to the $\operatorname{IrCC_{1\times 1}}$ scheme at an FER of $44410^{-5}$, where $\operatorname{IrCC} C_{T \times R}$ represents the IrCC-URC-STTC scheme 445 and the subscript ' $T \times R$ ' indicates having $T$ transmit and $R$ 446 receive antennas. Hence, for the sake of guaranteeing the same 447 FER performance, $\operatorname{IrCC}_{2 \times 2}$ exhibits a larger transmit range at 448 a given transmit power and may hence potentially reduce the 449 number of hops required for conveying a message from the 450 source to the destination, which can be explained by analyzing 451 the calculation of the transmission range. More explicitly, the 452 average maximum transmission range is defined as the range, 453 over which the receiver node is capable of receiving a transmit454 ted packet with $F E R<10^{-5}$.

455 The required minimum signal-to-noise ratio $S N R_{d B}^{*}$ may be 456 calculated from the minimum receive power $P_{r}^{*}$ expressed in $457 \mathrm{dBm}$ as follows

$$
S N R_{d B}^{*}=10 \log _{10}\left(\frac{P_{r}^{*}}{N_{0}}\right)
$$

(a)

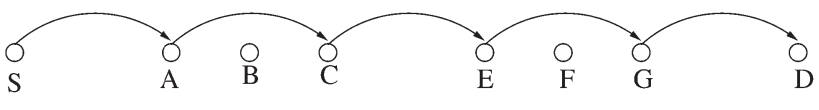

(b)

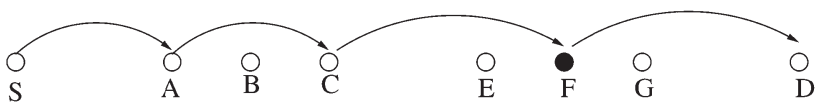

(c)

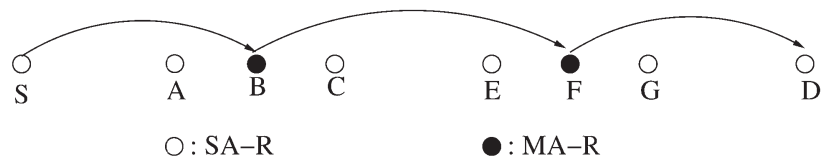

Fig. 8. The influence of MA-RNs on the routing strategy: (a) $H=5$ hops without MA-RNs; (b) $H=4$ with $1 \mathrm{MA}-\mathrm{RN}$ at point $F$; (c) $H=3$ with 2 MARNs at points $B$ and $F$.

where $N_{0}$ is the thermal noise power. Hence, given the transmit- 458 ted power $P_{t}$ and $S N R_{d B}^{*}$, the average maximum distance $d_{\max } 459$ from the transmitter, where the SNR requirement $S N R_{d B}^{*}$ may 460 'just' be satisfied to guarantee $F E R<10^{-5}$, is given by

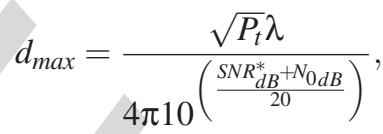

where the carrier's wavelength $\lambda=c / f$ and $N_{0 d B}=10 \log _{10} N_{0} .462$ $c$ is the speed of light in vacuum and $f$ is the carrier frequency. 463

Naturally, if the value of $P_{t}$ and $N_{0 d B}$ are fixed, then it may be 464 readily seen how the adequately 'illuminated' distance, where 465 the required target-FER may be maintained, will vary as a 466 function of the SNR value. As seen from Fig. 7, the maximum 467 adequately covered communication distance from MA-RN to 468 MA-RN is the highest, while that from SA-RN to SA-RN is the 469 lowest. Conversely, if $P_{t}$ and $d_{\max }$ are fixed, then the FER is the 470 lowest for the MA-RN to MA-RN link, while it is the highest 471 for the SA-RN to SA-RN link.

\section{B. Routing Algorithms}

It was shown in [77] that the IrCC-URC-STTC scheme is 474 capable of operating near the link's capacity, hence a substantial 475 power saving may be attained. When this scheme is employed 476 by the MA-RNs of the ad hoc network considered, the different 477 error correction capability of the four different types of links 478 will influence the routing strategy. Fig. 8 provides an example 479 on how the routing strategy is influenced.

As seen from Fig. 8, the network consists of $N=8$ nodes, 481 where $S$ is the source and $D$ is the destination. In Fig. 8(a), all 482 nodes are equipped with a single antenna, hence all links are 483 SA-RN to SA-RN links, which yields $H=5$ hops from $S$ to $D .484$ A single MA-RN is employed at point $F$ in Fig. 8(b), where the 485 packets arriving at node $C$ are directly transmitted to node $F .486$ Then, node $F$ will forward its received packets further to the 487 destination $D$. More specifically, the $C$-to- $F$ link is a SA-RN 488 to MA-RN link, while the $F$-to- $D$ link is an MA-RN to $D$ link, 489 where the $F-D$ distance is higher than that between the single- 490 antenna nodes of Fig. 8(a). Consequently, the number of hops 491 from $S$ to $D$ is decreased to $H=4$. In Fig. 8(c), two MA-RNs, 492 namely $B$ and $F$, are employed. The number of hops is further 493 decreased to $H=3$ as a benefit of using MA-RNs for nodes $B 494$ and $F$. 


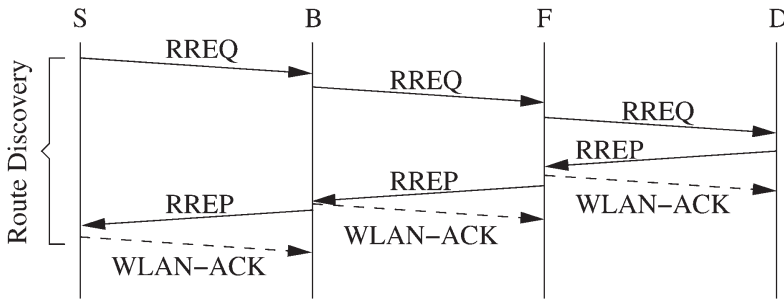

Fig. 9. The process of route discovery in the DYMO routing algorithm.

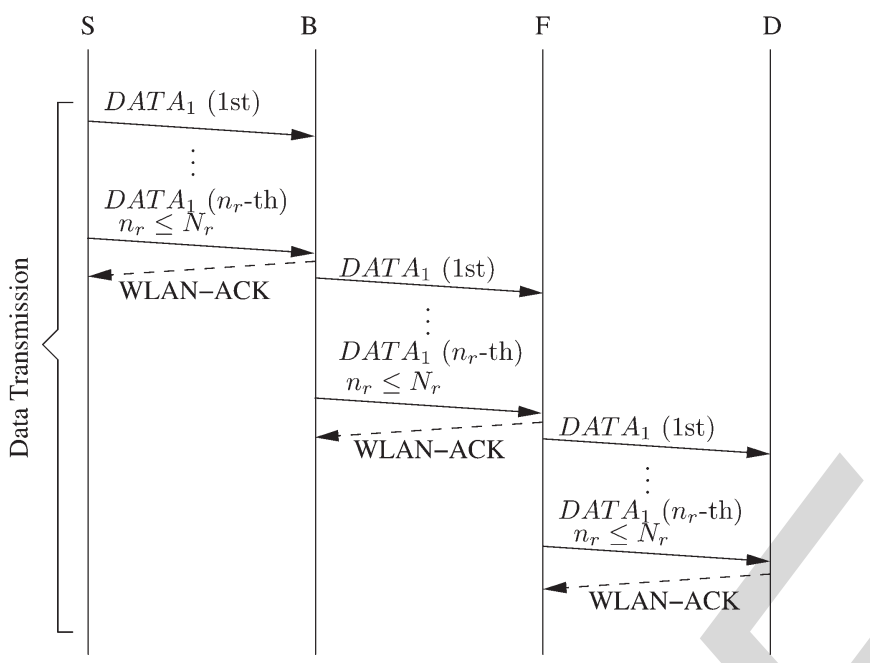

Fig. 10. The process of data transmission after a route is found from source $A$ to destination $D$.

496 The DYMO routing protocol is employed in the NET layer, 497 which combines most of the benefits of the AODV [33] and 498 DSR [32] protocols. The DYMO routing protocol always opts 499 for the specific route having the lowest number of hops to the 500 destination. When employing the MA-RN aided IrCC-URC501 STTC scheme, it will be demonstrated that the route selected 502 may be expected to have a further reduced number of hops. 503 The DYMO routing protocol is constituted by two main stages, 504 namely the route discovery and route maintenance. During the 505 route discovery, the Route REQuest (RREQ) and the Route 506 REPly (RREP) packets are used for identifying a route from 507 the source to the destination. By contrast, during the route 508 maintenance phase, a Route ERRor (RERR) packet is returned 509 to the source, when a broken link is detected. Figs. 9-11 show 510 the process of route discovery and data transmission as well 511 as route maintenance for the DYMO routing protocol, which 512 assisted us in analyzing the total energy consumption of the 513 system. The topology considered in Figs. 9-11 has a source $S$, 514 a destination $D$ and the pair of RNs $B$ and $F$. It is assumed that 515 each node is only capable of communicating with its neighbour 516 nodes. For example, node $B$ can only communicate with node $517 S$ and node $F$, while it cannot communicate with node $D$. The 518 exchange of the control packets between the neighbour nodes, 519 such as the exchange of the RREQ packet, RREP packet and 520 RERR packet, and the associated data transmission process is 521 detailed as follows:

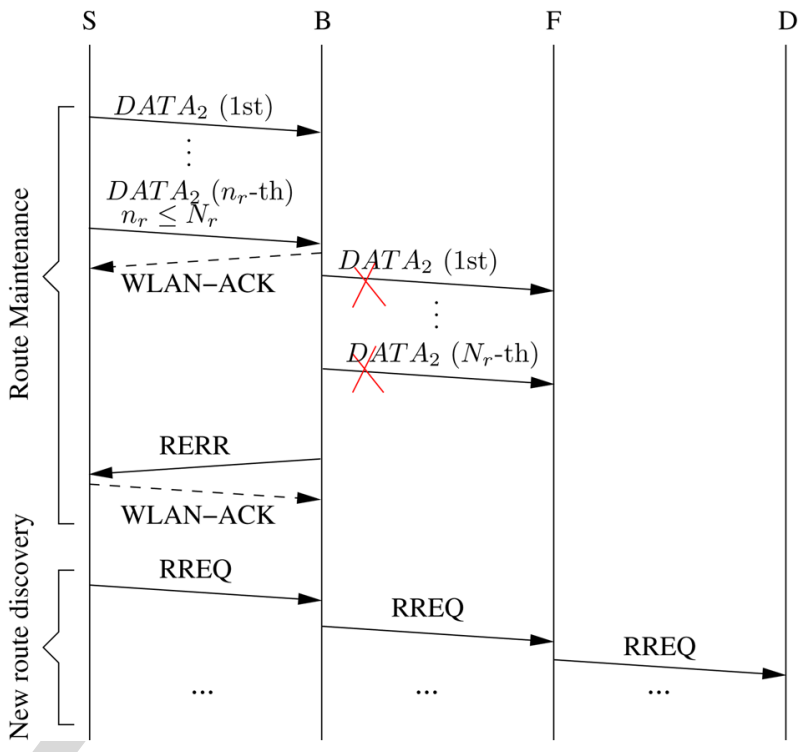

Fig. 11. The process of route maintenance in the DYMO routing algorithm.

As seen in Fig. 9, first the source $S$ broadcasts an RREQ 523 packet and when node $B$ receives this RREQ packet, it 524 broadcasts it. Then node $F$ receives the RREQ packet and 525 broadcasts it again. Finally, the destination $D$ receives the 526 RREQ packet, which originated from the source $S$; The 527 destination $D$ responds to the RREQ packet with a newly 528 generated RREP packet. The routing table of each node 529 is refreshed, when ever an RREQ/RREP packet arrives 530 at a node. Additionally, a Wireless Local Area Network- 531 Acknowledgement (WLAN-ACK) packet $^{1}$ is required for 532 confirming the successful reception of the RREP packet. 533

- Data Transmission process of Fig. 10.

534

When the RREP packet arrives at the source $S$ during 535 the process of route discovery, the source $S$ is informed 536 of a route spanning from the source $S$ to the destination 537 $D$, with node $B$ being the next hop of this route. Hence, 538 as seen in Fig. 10, the buffered data packet $D A T A_{1}$ is 539 transmitted to node $B$ according to the routing information 540 stored in the routing table of source $S$. If the packet $D A T A_{1} 541$ failed to reach node $B$, then node $B$ has to retransmit 542 the packet $D A T A_{1}$ until the number of retransmission 543 reaches its maximum of $N_{r}$. If and only if node $B$ receives 544 the packet $D A T A_{1}$ successfully within $n_{r}$ retransmissions, 545 where $n_{r} \leq N_{r}$, it would respond to source $S$ by sending 546 back a WLAN-ACK packet. The WLAN-ACK is used 547 for confirming the successful transmission of the packet 548 $D A T A_{1}$. Meanwhile, node $B$ forwards the packet $D A T A_{1}$ to 549 node $F$, since node $F$ is its next hop en route to destination 550 $D$. The routing information stored in node $B$ 's routing table 551 is obtained during the route discovery process as well. In 552 a similar way, if node $F$ successfully receives the packet 553 $D A T A_{1}$, it respond with a WLAN-ACK to node $B$ and 554

\footnotetext{
${ }^{1}$ The Acknowledgement packet is the one, which is returned to the transmitter as the acknowledgement of the correctly received data in the DL layer, hence it is referred to as WLAN-ACK in this treatise, where 'WLAN-ACK' represents the ACK packet employed in the IEEE802.11 standard. It is assumed that no Request-To-Send (RTS)/Clear-To-Send (CTS) mechanism is employed.
} 

586 Consequently, the sum of the energy $E_{T}$ dissipated by all nodes 587 in the network is given by

$$
\begin{aligned}
E_{T}= & \sum E_{\text {Route_Discovery }}+\sum E_{\text {Data_transmission }} \\
& +\sum E_{\text {Route_Maintenance }},
\end{aligned}
$$

588 where $E_{T}$ indicates the energy dissipated by a specific network 589 topology. $\sum E_{\text {Route_Discovery }}$ denotes the sum of energy dissi590 pated by the RREQ, the RREP and the WLAN-ACK packets 591 during the route discovery phase, which is shown in Fig. 9. 592 Furthermore, $\sum E_{\text {Route_Maintenance includes all the energy during }}$ 593 the route maintenance phase, except for $\sum E_{\text {Data_Transmission, }}$ 594 which is the energy dissipated by the data packets and by 595 the corresponding WLAN-ACK packets, as shown in Figs. 10 596 and 11.

\section{C. System Analysis}

598 The overall energy consumption $E_{T}$ of the entire network is 599 dependent on numerous parameters, such as the node density $600 \rho$, the number of MA-RNs $n_{M A}$, the mobile speed, the number 601 of hops $H$ of the selected route and the amount of bits $L_{a p p}$ 602 received in the application layer of the destination. To reduce 603 the dimensionality of the investigations when characterizing 604 the benefits of MA-RNs on the node's achievable transmission 605 range and FER performance, the node density $\rho$, the mobile 606 speed and $L_{a p p}$ are assumed to be constant, then $E_{T}$ is further 607 normalized by $L_{a p p}$ and $N$ of the entire network, where $N$ is re-

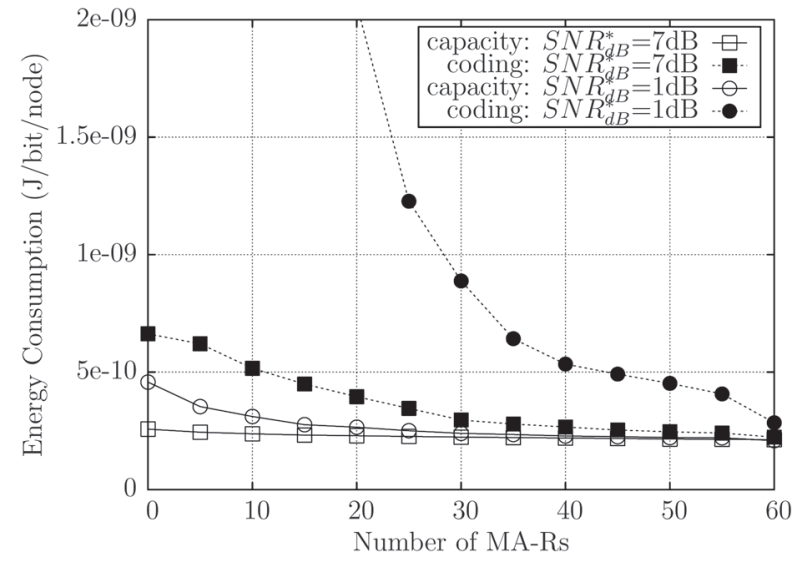

Fig. 12. Energy consumption $\bar{E}_{T}$ versus the number of MA-RNs $n_{M A}$ aiming for comparing the IrCC-URC-STTC scheme and the DCMC-capacity-based benchmark scheme at $S N R_{d B}^{*}$ of $7 \mathrm{~dB}$ and $1 \mathrm{~dB}$, where 'coding' denotes the IrCC-URC-STTC scheme and 'capacity' represents the DCMC-capacity-based benchmark scheme.

TABLE IV

SYSTEM PARAMETERS

\begin{tabular}{|l|r|}
\hline \hline Path-loss exponent & 2 \\
\hline Sensitivity threshold [82], $P_{r}^{*}$ & $-85 \mathrm{dBm}$ \\
\hline Mobility & stationary $(0 \mathrm{~m} / \mathrm{s})$ \\
\hline Simulation time & 30 second \\
\hline Number of simulation runs & 100 \\
\hline \hline
\end{tabular}

lated to the node density. Finally, the Normalized Energy Con- 608 sumption (NEC) $\bar{E}_{T}$ of the entire network can be expressed as: 609

$$
\bar{E}_{T}=\frac{E_{T}}{N L_{a p p}}=F\left(n_{M A}, H\right) .
$$

Four scenarios are considered to study the relationship be- 610 tween the number of MA-RNs and the energy consumption, 611 where $N=60$ stationary nodes are uniformly located in a 612 $500 \mathrm{~m} \times 500 \mathrm{~m}$ field, hence the node density is $\rho=240$ nodes 613 per square kilometer. The source $S$ and the destination $D$ are 614 located in the position $(499,499)$ and $(0,0)$, respectively. The 615 number of MA-RNs ${ }^{2}$ is increased from $n_{M A}=0$ to $n_{M A}=60616$ in steps of 5. The frame length of the data packets, which are 617 generated by the application layer, is $L_{a p p}=504$ Bytes. The 618 802.11b standard is employed in the DL layer. The transmit 619 power is set to $P_{T}=1 \mathrm{~mW}$. The other system parameters 620 employed for the simulations of Fig. 12 are listed in Table IV, 621 where the receiver's sensitivity [82] threshold is used to judge 622 whether the received signal is deemed to be noise, because a 623 received signal power below the sensitivity level is deemed to 624 be noise.

The energy consumption is quantified for both the IrCC- 626 URC-STTC scenario ${ }^{3}$ and the DCMC-capacity-based bench- 627 mark scenario at $S N R_{d B}^{*}$ of $7 \mathrm{~dB}$ and $1 \mathrm{~dB}$. As seen from 628 Fig. 12, the energy consumption of the IrCC-URC-STTC 629 scheme and of the benchmark scheme decrease upon increasing 630

\footnotetext{
${ }^{2}$ The number of MA-RNs also includes the source and the destination. Again, the multi-antenna aided nodes are denoted as MA-RNs and single-antenna nodes are denoted as SA-RNs.

${ }^{3}$ In the IrCC-URC-STTC scenario the network consists of QPSK-assisted IrCC-URC-STTC aided MA-RNs and 8PSK-assisted IrCC-URC aided SA-RNs.
} 
631 the number of MA-RNs $n_{M A}$. As mentioned in Section II-A, 632 a low FER and a relatively high transmission range i.e. cov633 erage area may be ensured by using the IrCC-URC-STTC 634 scheme advocated. Furthermore, as justified in Section II-B, 635 the specific routes having the lowest number of hops tend to be 636 activated in the MA-RNs aided network considered. Therefore, 637 having a high PHY-layer FER results in an increased number 638 of retransmissions and hence may trigger route re-discovery, 639 which results in more control packets being transmitted. Hence, 640 more energy per payload bit is required for successfully de641 livering the source data to the destination, as demonstrated 642 in Fig. 12.

\section{3 \\ 644 \\ III. Routing Design With PHY \& DL \& NET COOPERATION}

645 In recent years, numerous energy-efficient techniques have 646 been proposed [26], [64], [65], [69], [83]-[106]. However, 647 simply minimizing the energy consumption results in deficient 648 designs. It is more beneficial to strike a tradeoff between the 649 energy consumed and other metrics, such as the attainable 650 throughput. For example, Multiple-Input and Multiple-Output 651 (MIMO) schemes and near-capacity Space-Time Codes (STCs) 652 were employed in [84] for optimizing the RN selection for 653 the sake of maximizing the end-to-end throughput at a given 654 total available power. While single-hop transmissions are more 655 suitable for bandwidth-limited scenarios, multi-hop transmis656 sions combined with spatial frequency-reuse tend to perform 657 better in power-limited situations [69]. Spatial frequency-reuse 658 employed in multi-hop scenarios may be beneficially com659 bined with Interference Mitigation (IM) [69], [84] and transmit 660 beamforming [84] for the sake of finding an attractive balance 661 between energy minimization and throughput maximization in 662 both single-hop and multi-hop schemes [69], [85], [86]. As a 663 further advance, a beneficial tradeoff between the total energy 664 consumption and throughput was found in [85] by considering 665 both the transmission strategy of each node as well as the 666 location of the RNs and the data rate of each node.

667 Moreover, the authors of [26], [57], [86], [89], [92], [95]668 [98], [101]-[104], [107] invoked cross-layer design. For ex669 ample, the impact of the link error rate on the route selection 670 between a path associated with a large number of short-distance 671 hops and another with a smaller number of long-distance hops 672 was studied in [86]. In this paper, the link 'cost' was defined as 673 a function of both the energy required for a single transmission 674 attempt and the link error rate. This Objective Function (OF) 675 captures the cumulative energy expended in reliable data trans676 fer for both reliable and unreliable link layers. In [107], several 677 routing algorithms were proposed, which opted for the route 678 with minimum energy consumption in a mixed hop-by-hop and 679 end-to-end retransmission mode. In the end-to-end retransmis680 sion mode, a single unreliable link may require retransmissions 681 from the source, and hence may require more energy for suc682 cessfully delivering packets. Consequently, routing protocols 683 play an important role in saving energy. The authors of [57] 684 took into account both the energy consumed by data packets as 685 well as by control packets and MAC retransmissions, because 686 ignoring the energy consumption of exchanging control packets might underestimate the actual energy consumption and thus 687 may lead to inefficient designs. However, the energy OFs em- 688 ployed in [57], [86], [107] exploited the assumption of having 689 access to a potentially infinite number of MAC retransmissions, 690 which is unrealistic. The employment of the OF proposed in 691 [57], [86], [107] is feasible only when the affordable number of 692 MAC retransmissions is infinite, which is formulated as

$$
E_{\text {total }}=\sum_{1}^{H} \frac{E_{i}}{1-F E R_{i}}
$$

where $\frac{1}{1-F E R_{i}}$ is the expected number of transmission at- 694 tempts required for successfully delivering a packet across 695 link $i$. As seen from (5), the total energy of all hops is simply 696 summed, which suggests that the success of the individual links 697 in a route is deemed to be independent of each other, since the 698 assumption that an infinite number of MAC retransmissions 699 is affordable is given. Additionally, although the authors of 700 [89] considered a limited number of MAC retransmissions, no 701 specific OF was formulated.

702

Furthermore, TR relies on a route discovery process invoked 703 for gleaning sufficient routing information for the source to 704 make meritorious routing decisions, regardless, whether the 705 routing protocol is proactive or reactive [108]. However, due to 706 the rapid fluctuation of the channel conditions, the routing in- 707 formation estimated on the basis of the average Channel Quality 708 Information (CQI) may become stale, resulting in suboptimum 709 routing. Therefore, OR [90]-[92], [96], [101], [103], [109]- 710 [114] has been proposed for avoiding this problem. In OR no 711 pre-selected route is employed, instead a so-called forwarder 712 $\mathrm{RN}$ set is used for forwarding the packets along a benefi- 713 cial route. The near-instantaneously varying characteristics of 714 wireless channels is beneficially exploited considered by OR. 715 Table V shows that OR is widely used in various networks, such 716 as ad hoc networks [103], [115], wireless sensor networks [91], 717 cognitive networks [116], vehicular networks [117], [118] and 718 DTNs [119]-[121].

More specifically, Liu et al. [110] illustrated the basic idea 720 behind OR and categorized the potential design criteria, includ- 721 ing the Estimated Transmission count (ETX), the geographic 722 distance aided and the energy consumption based philosophies. 723 Biswas and Morris [101] proposed an Extremely Opportunistic 724 Routing (ExOR) scheme, which employed the ETX metric at 725 the destination for deciding the priority order of selecting a 726 $\mathrm{RN}$ from the potential forwarder set. The proposed routing 727 regime integrated the routing protocol and the MAC protocol 728 for the sake of increasing the attainable throughput of multi- 729 hop wireless networks. Their solution [101] also exploited 730 the less reliable long-distance links, which would have been 731 ignored by traditional routing protocols. Moreover, Dubois- 732 Ferrière et al. [111] conceived the Least-Cost Anypath Routing 733 (LCAR) regime, which finds the optimal choice of candidate 734 RNs relying on the expected ETX cost of forwarding a packet 735 to the destination. This LCAR algorithm considers the coordi- 736 nation of the link layer protocols. Laufer et al. [114] proposed 737 a 'polynomial-time multirate anypath' routing algorithm and 738 provided the proof of its optimality. The proposed routing 739 algorithm employed the Expected Anypath Transmission Time 740 
TABLE V

OpPortunistic Routing Protocols in VARIOUs Networks

\begin{tabular}{|c|c|c|}
\hline Year & Authors & Contribution \\
\hline 2006 & Pelusi et al. [117] & $\begin{array}{l}\text { Surveyed the most promising OR solutions and the taxonomy of the main routing and forwarding } \\
\text { approaches in challenging environments. }\end{array}$ \\
\hline 2008 & Conan et al. [121] & Proposed a single copy and multi-hop OR scheme for sparse Delay Tolerant Networks (DTNs). \\
\hline \multirow[t]{2}{*}{2009} & Khalife et al. [118] & $\begin{array}{l}\text { Explored opportunistic forwarding without preestablished routing in multihop cognitive radio } \\
\text { networks. }\end{array}$ \\
\hline & Spyropoulos et al. [122] & $\begin{array}{l}\text { Proposed a class of routing schemes that can identify the nodes of "highest utility" in intermittently } \\
\text { connected wireless networks. }\end{array}$ \\
\hline \multirow[t]{2}{*}{2010} & Lee et al. [119] & $\begin{array}{l}\text { Presented a topology-assisted Geo-OR designed for vehicular networks that combined topology- } \\
\text { assisted geographic routing with opportunistic forwarding for mitigating the effects of unreliable } \\
\text { wireless channels. }\end{array}$ \\
\hline & Li et al. [123] & $\begin{array}{l}\text { Investigated energy-efficient opportunistic forwarding and designed different forwarding policies for } \\
\text { DTNs. }\end{array}$ \\
\hline 2011 & Mao et al. [91] & $\begin{array}{l}\text { Presented an energy-efficient OR strategy conceived for wireless sensor networks, which created a } \\
\text { prioritized forwarder list to minimize the total energy consumption of all nodes. }\end{array}$ \\
\hline 2012 & Wang et al. [103] & $\begin{array}{l}\text { Proposed a cooperative OR scheme for mobile ad hoc networks for tackling the problem of } \\
\text { opportunistic data transfer. }\end{array}$ \\
\hline 2013 & Wu et al. [120] & $\begin{array}{l}\text { Proposed a hybrid routing scheme for data dissemination in vehicular ad hoc networks (VANETs), } \\
\text { using a carry-and-forward scheme for mitigating the forwarding disconnection } \\
\text { problem of sparse VANETs. }\end{array}$ \\
\hline 2014 & Yoon et al. [124] & $\begin{array}{l}\text { Investigated the feasibility of OR in power line communications access networks and proposed a } \\
\text { customized OR, which used static geographical information. }\end{array}$ \\
\hline
\end{tabular}

741 (EATT) as the routing metric, which is a generalization of the 742 unidirectional ETX metric that takes into account that nodes 743 transmit at multiple bit rates. The authors of [90], [109], [113] 744 employed a geographic distance based metric for choosing the 745 potential forwarder RN set. More specifically, Zorzi and Rao 746 [109] proposed an OR scheme based on random forwarding, 747 where the specific node, which is closest to the destination 748 is chosen as the RN for the next hop. Additionally, they [90] 749 analyzed the achievable energy as well as latency performance 750 and provided a detailed description of a MAC scheme based 751 on both opportunistic concepts and on collision avoidance. 752 Zeng et al. [113] proposed a multirate OR by incorporating 753 rate adaptation into their candidate-selection algorithm, which 754 was shown to achieve a higher throughput and lower delay 755 than the corresponding traditional single-rate routing and its 756 opportunistic single-rate routing counterpart. The authors of 757 [91], [92], [96] employed the energy consumption metric for 758 choosing the potential forwarder RN set. More concretely, 759 Mao et al. [91] presented an energy-efficient OR strategy 760 relying on sophisticated PA, which prioritizes the forwarder 761 RNs by directly minimizing the total energy consumption of 762 all nodes. Dehghan et al. [92] developed a minimum-energy 763 cooperative routing based on many-to-many cooperation and 764 determines the optimal route with the aid of the Bellman-Ford 765 algorithm [123]. Wei et al. [96] proposed an energy-conserving 766 Assistant Opportunistic Routing (AsOR) protocol, which clas767 sified a sequence of nodes into three different node sets, namely, 768 the frame node, the assistant node and the unselected node. 769 The frame nodes were indispensable for decode-and-forward 770 operation, while the assistant nodes provided protection against 771 unsuccessful opportunistic transmissions. Although the authors 772 of [91], [92], [96] employed the energy consumption as their 773 routing metric, they have not provided any theoretical bounds 774 in their performance analysis. Moreover, these authors as775 sumed that the number of affordable MAC retransmissions 776 was infinite.

777 An appropriate PA scheme combined with an opportunistic 778 scheme was introduced in [74]. The opportunistic scheme does

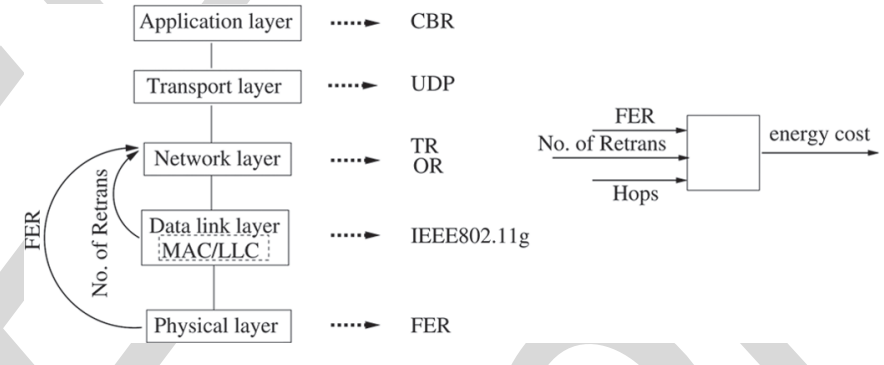

Fig. 13. System model of the energy-efficient routing with PHY \& DL \& NET cooperation in ad hoc networks.

not employ a pre-selected route, while it will fully utilize 779 the time-variant characteristic of the hostile wireless channel, 780 where any RN has the chance to forward a packet as long as 781 the packet arrives at this RN successfully. A pair of energy- 782 consumption-based OFs are constructed for TR and OR by 783 exploiting the knowledge of both the corresponding FER within 784 the PHY layer, as well as that of the number of MAC retrans- 785 missions and of the number of RNs in the NET layer, as seen 786 in the system model of Fig. 13. The above-mentioned TR and 787 OR algorithms employ the corresponding energy-consumption- 788 based OFs as their routing metrics, respectively. Apart from 789 the energy consumption, the end-to-end throughput is evaluated 790 as well. It was demonstrated that the algorithms proposed in 791 [74] are capable of operating close to the theoretical bound 792 found by the exhaustive search of all routes. In Fig. 13, the 793 characteristics of the PHY layer are represented with the aid 794 of the FER, while the DL layer employs the IEEE802.11g 795 standard. In the NET layer, the above-mentioned TR and OR 796 are employed, which make their decisions on the basis of the 797 above energy-consumption-related OFs. The UDP is employed 798 in the transport layer and the data streaming relies on a CBR 799 service in the application layer. As in Section II, the channel 800 imposes both free-space path-loss and uncorrelated Rayleigh 801 fading, plus the ubiquitous AWGN.

802

Based on the system model of Fig. 13, the impact of the 803 lowest three layers of the OSI model on the total energy 804 
805 dissipated of the entire system is considered, which will be 806 analyzed, whilst relying on an energy-consumption-based OF. 807 In [73], [74], only the transmit energy consumed by the 808 data packets during their transmission is considered, which are 809 generated by the application layer. The energy consumed by 810 other packets, such as routing and MAC control packets is not 811 considered. In other words, the idealized simplifying assump812 tion is that the energy consumed during the process of route 813 discovery is negligible. The elimination of this simplification 814 was set aside for the future work. As detailed in [73], [74], 815 the $\mathbf{O F}$ is invoked for making routing-related decisions, which 816 directly influence the energy consumed by future data packets. 817 All nodes are assumed to be stationary. Only a single source818 destination pair is supported in the network and only a single 819 node has the chance of transmitting in a time slot, once the route 820 was determined. All the data packets are also assumed to have 821 the same length and all nodes have the same transmission rate.

\section{A. Traditional Routing With Fixed Transmit Power}

823 Naturally, having an infinite number of MAC retransmis824 sions will impose a potentially infinite end-to-end delay at the 825 destination, which is not realistic. In realistic environments, 826 the wireless link may become broken owing to packet errors 827 if the maximum number of MAC retransmissions has been 828 exhausted. A broken link may trigger a route-repair or even 829 route re-discovery for the sake of maintaining the current 830 source-destination communications session. The route-repair 831 is often required at the upper-node's broken link, while the 832 route re-discovery should be initiated by the source. All these 833 actions may consume more energy and naturally they reduce 834 the attainable throughput. Additionally, the success of a specific 835 hop emanating from a node relies on the success of all previous 836 hops. If any of the previous links is broken, then no packet 837 will be forwarded towards the destination. Naturally, any link 838 is more likely to break if the number of MAC retransmissions 839 is limited to $N_{r}$. The energy consumption considered is divided 840 into two parts: the energy consumed by the data packets which 841 succeed in reaching the destination and the energy consumed 842 by the data packets which are dropped before reaching the 843 destination. The time slot duration of a single transmission 844 attempt across a given link is defined as $T$. Given the same data 845 packet length and the same transmission rate at each node, $T$ is 846 a constant value. Here, the energy-conscious OF of a two-hop 847 route is detailed as an example. $p_{s}$ and $p_{f}$ are used to denote 848 the probability of a packet being successfully delivered to the 849 destination successfully and being dropped before reaching the 850 destination, respectively. Furthermore, the notation $p_{s}(\tau)$ repre851 sents the probability that the packet is successfully delivered 852 all the way from the source to the destination after a time 853 duration of $\tau$. First, the energy consumption analysis of a 2-hop 854 route is considered in Fig. 14. The symbol $\sqrt{ }$ indicates that 855 the link's transmission is successful after $1 \leq \frac{\tau}{T} \leq N_{r}$ MAC 856 retransmission attempts. Hence the time duration of the link's 857 transmission is $T \leq \tau \leq N_{r} T$. Fig. 14 shows that a packet's 858 successful transmission over the link $S-R_{1}$ requires a time 859 duration of $\tau_{1}$, while the successful transmission of a packet 860 over the link $R_{1}-D$ requires a time duration of $\tau_{2}$. Hence the

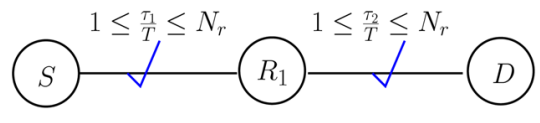

Fig. 14. A packet is successfully delivered from $S$ to $D$ in a 2-hop route.

(a)
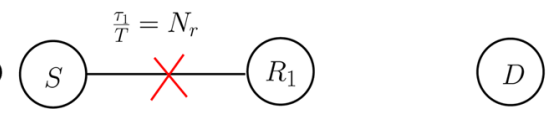

(b)

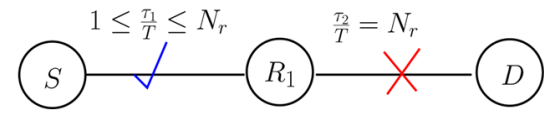

Fig. 15. A packet is dropped before reaching $D$ in a 2-hop route.

total time duration of a packet's passage between $S$ and $D$ is 861 $\left(\tau_{1}+\tau_{2}\right)$, where $2 T \leq \tau_{1}+\tau_{2} \leq 2 N_{r} T$.

Therefore,

$$
\begin{aligned}
p_{s}(2 T)= & p_{1} p_{2}, \\
p_{s}(3 T)= & \left(1-p_{1}\right) p_{1} p_{2}+p_{1}\left(1-p_{2}\right) p_{2}, \\
p_{s}(4 T)= & \left(1-p_{1}\right)^{2} p_{1} p_{2} \\
& +\left(1-p_{1}\right) p_{1}\left(1-p_{2}\right) p_{2}+p_{1}\left(1-p_{2}\right)^{2} p_{2}, \\
\vdots & \vdots \\
p_{s}\left(2 N_{r} T\right)= & \left(1-p_{1}\right)^{N_{r}-1} p_{1}\left(1-p_{2}\right)^{N_{r}-1} p_{2} .
\end{aligned}
$$

While $p_{s}$ is given by

$$
\begin{aligned}
p_{s} & =p_{s}(2 T)+p_{s}(3 T)+p_{s}(4 T)+\cdots+p_{s}\left(2 N_{r} T\right), \\
& =\sum_{1}^{N_{r}} \sum_{1}^{N_{r}}\left(1-p_{1}\right)^{i_{1}-1} p_{1}\left(1-p_{2}\right)^{i_{2}-1} p_{2} .
\end{aligned}
$$

Since during a single time slot $T$ the nodes consume an energy 865 of $E$, the estimated total energy $E_{s}$ consumed by a successfully 866 delivered packet in a two-hop route is

$$
E_{s}=\left[2 p_{s}(2 T)+3 p_{s}(3 T)+4 p_{s}(4 T)+\cdots+2 N_{r} p_{s}\left(2 N_{r} T\right)\right] E .
$$

In a similar way, the time $D_{s}$ required for a packet, which is 868 successfully delivered from $S$ to $D$ is given by

$$
D_{s}=\left[2 p_{s}(2 T)+3 p_{s}(3 T)+4 p_{s}(4 T)+\cdots+2 N_{r} p_{s}\left(2 N_{r} T\right)\right] T .
$$

Additionally, the packets, which exhausted the maximum 870 number $N_{r}$ of MAC retransmissions and were finally dropped 871 before reaching $D$ due to poor channel conditions also consume 872 energy. This energy should also be taken into account in the 873 total energy consumption. The energy dissipation analysis of a 874 packet dropped before reaching the destination in a 2-hop route 875 is portrayed in Fig. 15. The symbol $\times$ indicates that the link's 876 transmission fails after $\frac{\tau}{T}=N_{r}$ MAC retransmission attempts. 877 As seen in Fig. 15, a transmission failure may occur either in 878 the $S-R_{1}$ link or in the $R_{1}-D$ link of a 2-hop route. Hence, 879 even when the data transmission in the $S-R_{1}$ link is successful 880 within the time duration of $T \leq \tau_{1} \leq N_{r} T$, the transmission 881 might fail in the $R_{1}-D$ link. 


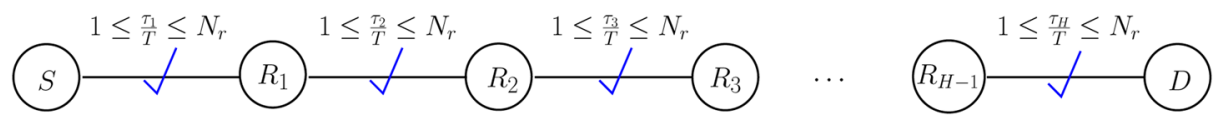

Fig. 16. A packet is successfully delivered from $S$ to $D$ in a $H$-hop route.

(a)

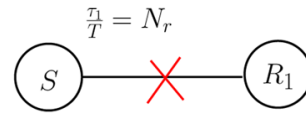

(b)

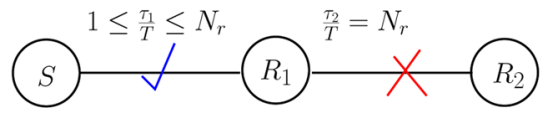

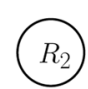
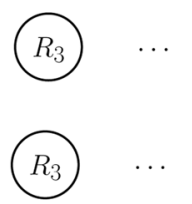

$\cdots$

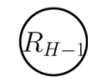

$R_{H-1}$

(c)
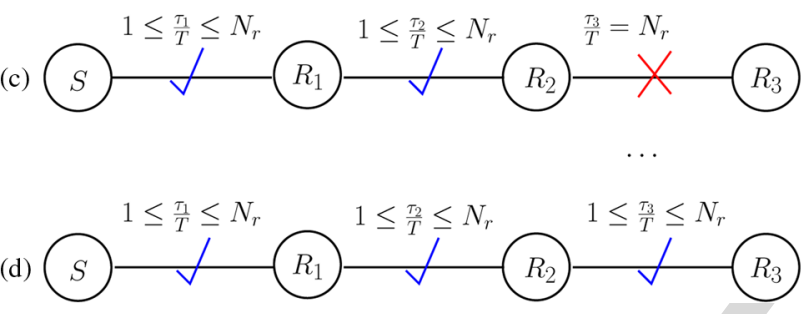
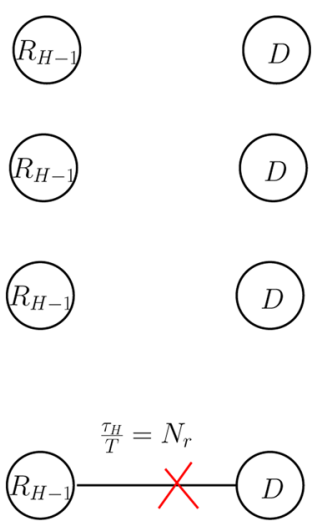

Fig. 17. A packet is dropped before reaching $D$ in a H-hop route.

883 The probability of failure $p_{f}$ of the two-hop route for a single 884 packet is described as follows:

$$
\begin{aligned}
p_{f}(1) & =\left(1-p_{1}\right)^{N_{r}}, \\
p_{f}(2) & =\left[\left(1-p_{f}(1)\right)\right]\left(1-p_{2}\right)^{N_{r}}, \\
p_{f} & =p_{f}(1)+p_{f}(2),
\end{aligned}
$$

885 where $p_{f}(h)$ represents the probability of the packet becoming 886 dropped during the $h$-th hop. Therefore, the energy $E_{f}$ con887 sumed by a dropped packet is quantified as follows:

$$
E_{f}=\left[N_{r} p_{f}(1)+\sum_{1}^{N_{r}}\left(1-p_{1}\right)^{i_{1}-1} p_{1}\left(1-p_{2}\right)^{N_{r}}\left(i_{1}+N_{r}\right)\right] E
$$

888 Similarly, the average time $D_{f}$ required by a packet to propagate 889 from $S$ up to the broken link is formulated as

$$
D_{f}=\left[N_{r} p_{f}(1)+\sum_{1}^{N_{r}}\left(1-p_{1}\right)^{i_{1}-1} p_{1}\left(1-p_{2}\right)^{N_{r}}\left(i_{1}+N_{r}\right)\right] T \text {. }
$$

890 The energy dissipation analysis of a packet's successful 891 delivery to the destination and that of a packet dropped before 892 reaching the destination in a $H$-hop route is characterized in 893 Fig. 16 and Fig. 17, respectively. Fig. 16 portrays the scenario, 894 where each link's transmission is successful after $1 \leq \frac{\tau}{T} \leq N_{r}$ 895 MAC retransmission attempts. By contrast, Fig. 17 shows that 896 a transmission failure could take place within any of the links, 897 where all the previous links' transmissions were successful. The 898 time duration elapsed before reaching the failed link is $\tau=N_{r} T$, 899 while that elapsing during all the previous link's transmission is $900 T \leq \tau \leq N_{r} T$.

901 Therefore, the total normalized transmit energy consumption 902 becomes:

$$
\bar{E}_{\text {total }}=\frac{E_{\text {total }}}{p_{s}}=\frac{E_{s}+E_{f}}{p_{s}} .
$$

Similarly, the end-to-end throughput $R_{e 2 e}$ is given as

$$
R_{e 2 e}=\frac{p_{s}}{D_{s}+D_{f}}
$$

A low-complexity routing algorithm is proposed in [73]. 904 The process of route discovery is shown in Fig. 18, where 905 $S$ represents the source, $D$ represents the destination, and the 906 other nodes are denoted by symbols $A, B, C, E, F$ and $G . E_{S \rightarrow n, t} 907$ denotes the estimated NEC for the route spanning from $S$ to 908 node $n$ at time instant $t$, while $E_{S \rightarrow n}$ is used for storing the 909 minimum NEC for every node in every time-slot of duration 910 $T$. The routing process may be divided into the following 911 four steps:

- Step 1 Node $S$ broadcasts the RREQ packet;

Step 2 Every node carries out the operations detailed in 914 Fig. 19 upon receiving the RREQ packet;

915

- Step 3 Node $S$ receives the RREP packet and then updates 916 the routing table;

- Step 4 Then node $S$ sends its data packet along the specific 918 route having the lowest estimated $\bar{E}_{\text {total }}$.

919

A flow chart is provided in Fig. 19 for specifically highlight- 920 ing the operations, when each node receives an RREQ packet. 921 If $S$ receives the RREQ packet, $S$ will simply discard this RREQ 922 packet. By contrast, if another node $n(n \neq S)$ receives the 923 RREQ packet, it calculates the NEC $E_{S \rightarrow n, t}$ and then compares 924 $E_{S \rightarrow n, t}$ to $E_{S \rightarrow n}$. If $E_{S \rightarrow n, t}>E_{S \rightarrow n}$, then node $n$ will discard the 925 RREQ packet. Otherwise, if node $n$ is $D$, then $D$ will respond 926 with a newly created RREP packet. However, if node $n$ is not 927 $D$, node $n$ will broadcast the RREQ packet again.

Now the process of routing discovery is explained in details 929 for further clarification. During time slot 1, node $S$ broadcasts 930 the RREQ packet, nodes $A, B$ and $C$ receive the RREQ packet. 931 According to the actions seen in Fig. 19, nodes $A, B$ and $C$ first 932 calculate $E_{S \rightarrow A, 1}, E_{S \rightarrow B, 1}$ and $E_{S \rightarrow C, 1}$, respectively. Then they 933 compare these newly calculated values with the aid of $E_{S \rightarrow A}, 934$ 
(a)

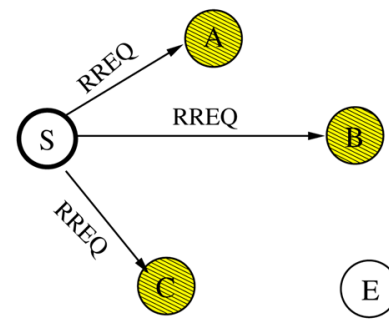

(c)

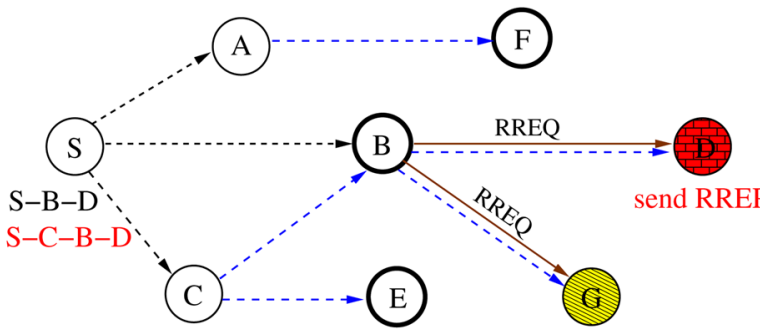

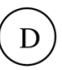

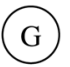

ready to forward RREQ

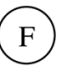

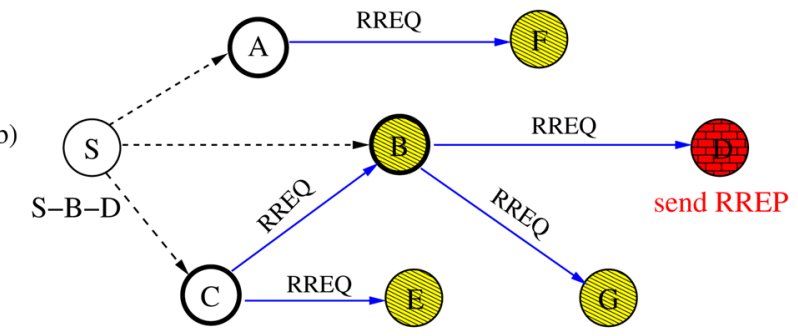

(d)

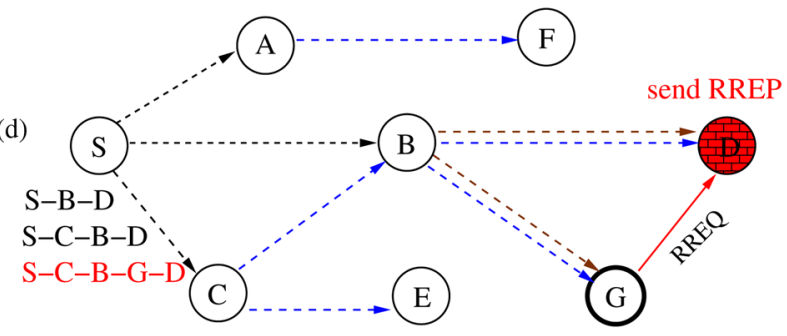

broadcast RREQ

ready to send RREP

$\longrightarrow E_{S \rightarrow n, t \leq E_{S \rightarrow n}}$ at current transmission - - $-E_{S \rightarrow n, t \leq E_{S \rightarrow n}}$ at previous transmission

Fig. 18. The process of route discovery in the low-complexity routing algorithm. (a) Actions during time slot 1 . (b) Actions during time slot 2 and node $S$ updates its routing table with the route $S-B-D$. (c) Actions during time slot 3 and node $S$ updates its routing table with the route $S-C-B-D$ since the estimated NEC of route $S-C-B-D$ is lower than that of route $S-B-D$. (d) Actions during time slot 4 and node $S$ updates its routing table with the route $S-C-B-G-D$, since the estimated NEC of route $S-C-B-G-D$ is lower than that of route $S-C-B-D$.

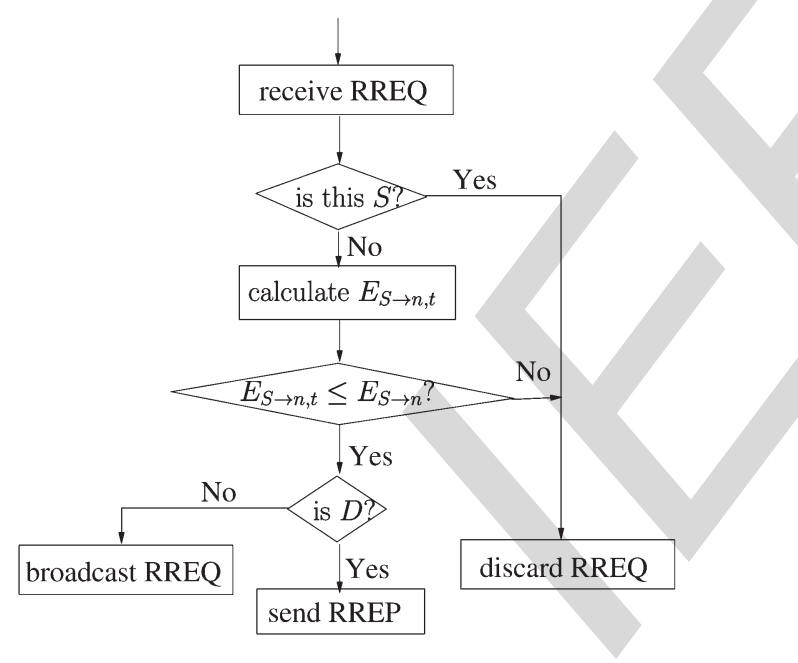

Fig. 19. Operations when each node receives an RREQ packet.

$935 E_{S \rightarrow B}$ and $E_{S \rightarrow A}$, respectively. If $E_{S \rightarrow A, 1} \leq E_{S \rightarrow A}$, then $A$ will 936 update $E_{S \rightarrow A}$ and will forward the RREQ packet during the 937 next time slot, otherwise it will discard it. The same actions 938 are carried out at node $B$ and node $C$ as well.

939 Then during time slot 2, node $A, B$ and $C$ forward the RREQ 940 packet. According to the actions seen in Fig. 19, nodes $B, E, F$ 941 and $G$ will forward the RREQ packet in the next time slot, since $942 E_{S \rightarrow B, 2} \leq E_{S \rightarrow B}, E_{S \rightarrow E, 2} \leq E_{S \rightarrow E}, E_{S \rightarrow F, 2} \leq E_{S \rightarrow F}$ and $E_{S \rightarrow G, 2} \leq$ $943 E_{S \rightarrow G}$. Node $D$ is ready to send back a newly created RREP 944 packet in the next time slot, since node $D$ is the destination and $945 E_{S \rightarrow D, 2} \leq E_{S \rightarrow D}$. Afterwards, $S$ will receive the RREP packet 946 and updates its routing table with the route $S-B-D$ obtained. $947 S$ will send the data packet along the route $S-B-D$.

948 During time slot 3, nodes $B, E, F$ and $G$ forward the RREQ 949 packet. According to the actions portrayed in Fig. 19, node $G$ 950 will forward the RREQ packet during the next time slot, since
$E_{S \rightarrow G, 3} \leq E_{S \rightarrow G}$. Node $D$ will then send back a newly created 951 RREP packet during the next time slot, since node $D$ is the 952 destination and $E_{S \rightarrow D, 3} \leq E_{S \rightarrow D}$. Afterwards, $S$ will receive the 953 second RREP packet and updates its routing table again with 954 the route $S-C-B-D$ obtained, since the estimated NEC of 955 route $S-C-B-D$ is lower than that of route $S-B-D$. Finally, 956 node $S$ will send the next data packet along the updated route 957 $S-C-B-D$.

958

During time slot 4 , node $G$ forwards the RREQ packet, 959 then nodes $B, E$ and $D$ receive it. According to the actions 960 of Fig. 19, then $D$ is ready to send back a newly created 961 RREP packet during the next time slot, since node $D$ is the 962 destination and $E_{S \rightarrow D, 4} \leq E_{S \rightarrow D}$. Afterwards, $S$ will receive the 963 third RREP packet and updates its routing table again with the 964 route $S-C-B-G-D$ obtained, since the estimated NEC of 965 route $S-C-B-G-D$ is lower than that of route $S-C-B-D .966$ Finally, node $S$ will send the next data packet along the updated 967 route $S-C-B-G-D$.

968

The analytically estimated NEC associated both with an 969 infinite number as well as a finite number of $N_{r}$ MAC re- 970 transmissions was calculated from (5) and (19), respectively. 971 A simple linear network topology was studied, where all $N 972$ nodes are equi-spaced along a line. The frame length of the 973 data packets, which are generated from the application layer, 974 is Lapp $=1024$ Bytes. The $802.11 \mathrm{~g}$ standard is employed in the 975 DL layer. The transmit power is set to $P_{t_{i}}=0.016 \mathrm{~mW}$ and the 976 IrCC-URC-QPSK defined in Section II is employed in the PHY 977 layer. The channel model is the AWGN channel subjected to 978 inverse second-power free-space path loss. The other system 979 parameters employed for the simulations of Figs. 20 and 21 are 980 listed in Table IV.

The NEC and the end-to-end throughput evaluated both from 982 (19) and (20) as well as by simulations are portrayed in Figs. 20983 and 21 . 


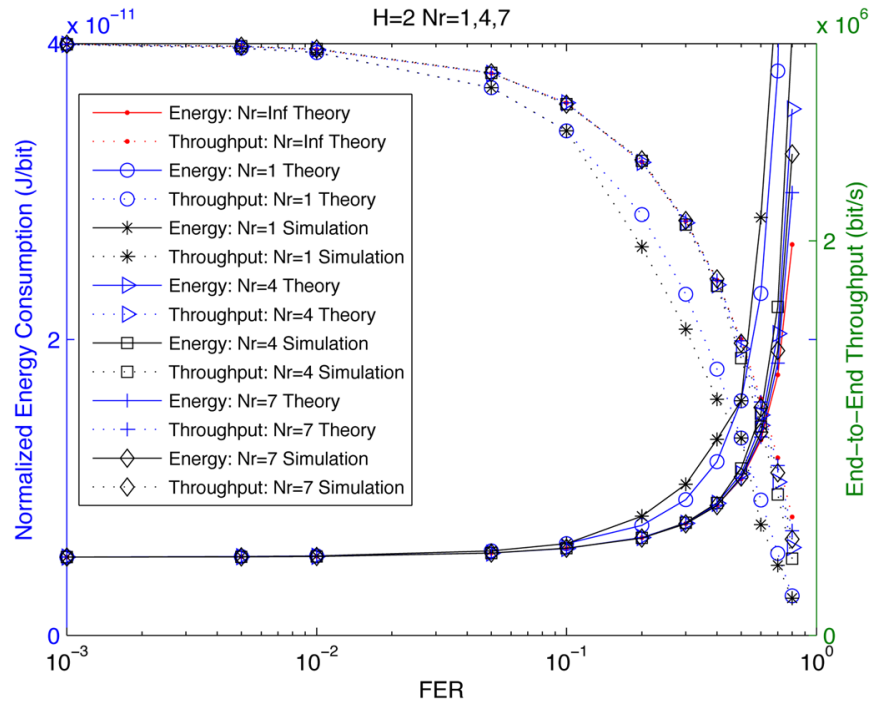

Fig. 20. The NEC and the end-to-end throughput versus FER $\left(10^{-3} \leq F E R \leq\right.$ 1) and the maximum number of MAC retransmissions $N_{r}\left(N_{r}=1,4,7\right)$ with the number of hops $H=2$ for comparing the theoretically analyzed values and simulation based values.

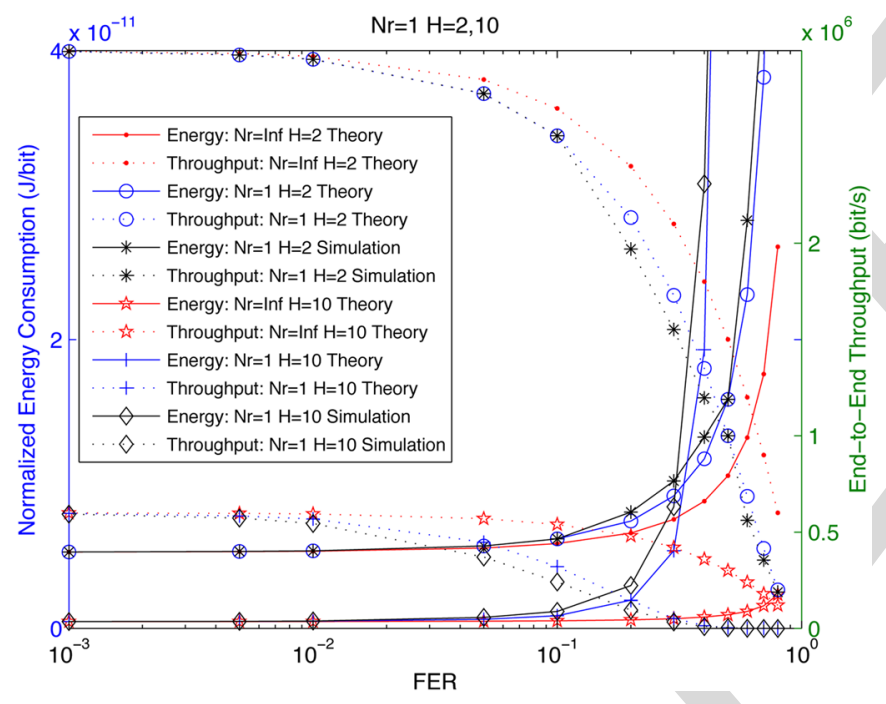

Fig. 21. The NEC and the end-to-end throughput versus FER $\left(10^{-3} \leq F E R \leq\right.$ 1) and the number of hops $H(H=2,10)$ with the maximum number of MAC retransmissions $N_{r}=1$ for comparing the theoretically analyzed values and simulation based values.

985 Fig. 20 displays three groups of performance curves recorded 986 at $N_{r}=1,4$ and 7, respectively, for both the NEC and for the 987 end-to-end throughput, where $N_{r}$ is the maximum number of 988 MAC retransmissions. The performance figures recorded for 989 the infinite number of MAC retransmissions scenario, namely 990 for $N_{r}=\infty$ are identical for the theory evaluated from (5) 991 and for the simulations. All the analytical and the simulation 992 based values recorded for the NEC increase, when the FER 993 increases. By contrast, the curves representing the end-to-end 994 throughput decrease, when the FER increases. The reason for 995 this observation is that a high FER in a link indicates a high 996 breakage probability not only for the specific link and but 997 also for the entire route, when retransmissions are required. 998 However, if $N_{r}$ is sufficiently high, then the success probability 999 of a packet across a link or even the entire route becomes higher.

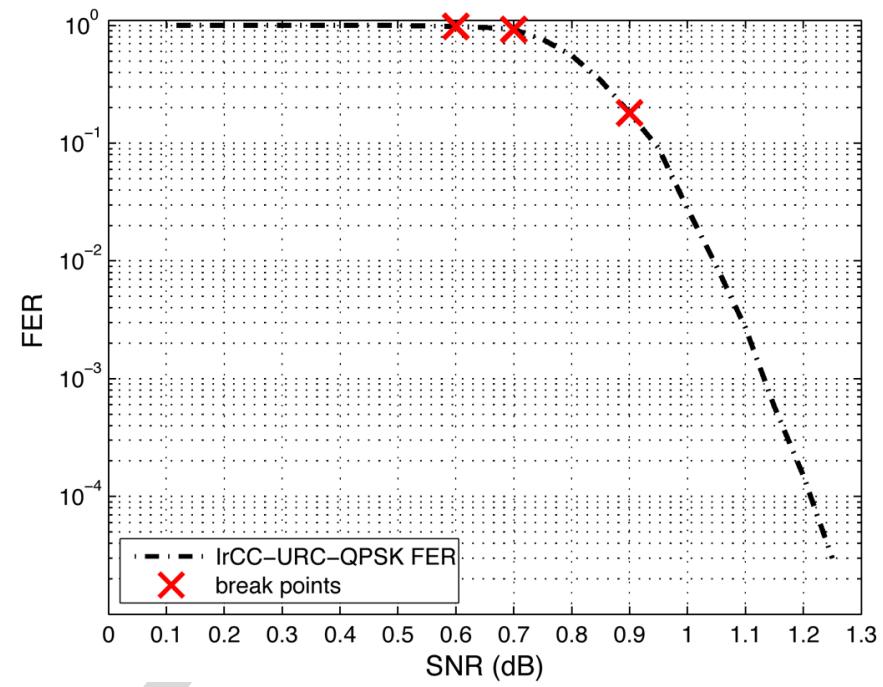

Fig. 22. FER versus SNR for the IrCC-URC-QPSK scheme of Section II-A for the average code rate of $R_{c}=0.5$ in an AWGN channel.

This trend is presented in Fig. 20, where the curve recorded for 1000 $N_{r}=7$ is seen to be close to that of $N_{r}=\infty$. The discrepancy 1001 between the theoretical value and the simulation-based value 1002 becomes higher when $N_{r}$ is reduced and simultaneously the 1003 FER is increased. Fig. 20 also shows that the theoretical energy 1004 consumption of (19) based on the energy-conscious OF is closer 1005 to the simulation based values than those based on the OF rely- 1006 ing on an infinite number of MAC retransmissions. Naturally, 1007 the advantage of the OF is more substantial for $N_{r}=1 . \quad 1008$

Fig. 21 also displays two groups of performance curves, 1009 one group for the NEC and the other group for the end-to- 1010 end throughput, which are associated with $H=2$ and 10, 1011 respectively. When $H$ is increased, the normalized energy 1012 consumption is reduced and the end-to-end throughput is de- 1013 creased, because the distance between a pair of adjacent nodes 1014 is reduced and therefore the transmit power required at each 1015 node for successfully delivering a packet is reduced. Similarly, 1016 as discussed in [73], the theoretical values estimated based 1017 on the proposed OF are closer to the simulated values than 1018 to those estimated on the basis of an infinite number of MAC 1019 retransmissions, especially when both $H$ and the FER are high. 1020

Hence, as elaborated on in [73], the conclusion is reached 1021 from Figs. 20 and 21 that the proposed energy-conscious OF 1022 is more accurate than the one assuming an infinite number of 1023 MAC retransmissions at high FERs, or for a high number of 1024 hops at a low maximum number of MAC retransmissions. 1025

\section{B. Traditional Routing With Adjustable Transmit Power}

1026

The FER curve was generated for the AWGN channel model 1027 with the aid of simulation [74]. According to the approach 1028 of [102], this will allow us to determine the average FER 1029 for arbitrary fading channels upon weighting the AWGN-FER 1030 by the Probability Distribution Function (PDF) of the fading 1031 channel and averaging it over the legitimate dynamic range. 1032 More specifically, the channel model considered is the uncor- 1033 related, non-dispersive Rayleigh fading channel. The average 1034 FER expression FER Rayleigh is determined for the Rayleigh 1035 fading channel considered by integrating the specific $F E R_{A W G N} 1036$ 
1037 value of the AWGN channel experienced at a given SNR after 1038 weighting it by the probability of that specific SNR, which is 1039 given by:

$$
F E R_{\text {Rayleigh }}=\int_{0}^{\infty} e^{-\gamma} F E R_{A W G N}(\gamma) d \gamma
$$

1040 where $\gamma$ is the channel SNR, $e^{-\gamma}$ represents the Rayleigh 1041 channel while the $F E R_{A W G N}(\gamma)$ versus the SNR curve is ap1042 proximated by the following four-segment FER vs. SNR model 1043 representing the AWGN channel:

$$
F E R_{A W G N}(\gamma) \approx \begin{cases}1, & \text { if } 0 \leq \gamma<\eta_{1}, \\ 10 a_{1} \log (\gamma)+a_{2}, & \text { if } \eta_{1} \leq \gamma<\eta_{2}, \\ 10 a_{3} \log (\gamma)+a_{4}, & \text { if } \eta_{2} \leq \gamma<\eta_{3}, \\ a_{5} e^{-10 a_{6} \log (\gamma)}, & \text { if } \gamma \geq \eta_{3},\end{cases}
$$

1044 with $\eta_{1}, \eta_{2}$ and $\eta_{3}$ being the break-points of the four-segment 1045 FER versus SNR approximation $F E R_{A W G N}(\gamma)$. Eqs. (21) and 1046 (22) are suitable for approximating different FER curves by ap1047 propriately setting the corresponding parameter values invoked. 1048 Eq. (21) may be readily extended to arbitrary channel models. 1049 Given $F E R_{\text {Rayleigh }}$, the successful reception probability of a 1050 link can be calculated as $\left[1-\left(F E R_{\text {Rayleigh }}\right)^{N_{r}}\right]$ if the maximum 1051 number of MAC retransmissions (including the first MAC 1052 retransmission attempt) is $N_{r}$. Specifically, for the IrCC-URC1053 QPSK scheme of Section II-A[72] employed, $a_{1}=-0.5889$, $1054 a_{2}=1.3341, a_{3}=-3.705, a_{4}=3.5169, a_{5}=4.4669 \times 10^{6}$ 1055 and $a_{6}=18.9118$. Additionally, the values of the break-points $1056 \eta_{1}, \eta_{2}$ and $\eta_{3}$ are determined for the SNR points of $0.6 \mathrm{~dB}$, $10570.7 \mathrm{~dB}$, and $0.9 \mathrm{~dB}$, which are based on the curves seen 1058 in Fig. 22. Fig. 22 shows the FER performance versus the 1059 SNR, when the IrCC-URC-QPSK scheme of Section II-A is 1060 employed, relying on the average code rate of $R_{c}=0.5$ in an 1061 AWGN channel. As seen from Fig. 22, the corresponding hori1062 zontal points of the symbol ' $x$ ' are $0.6 \mathrm{~dB}, 0.7 \mathrm{~dB}$ and $0.9 \mathrm{~dB}$. 1063 Therefore, by employing a practical coding scheme, such as 1064 the IrCC-URC-QPSK scheme of Section II with the aid of (19), 1065 it arrives at

$$
\bar{E}_{\text {total }}=\frac{P_{t_{1}}}{p_{1}} T
$$

1066 which shows that $\bar{E}_{\text {total }}$ is independent of the number of retrans1067 missions in a single-hop route. In this context, the NEC is the 1068 same as that of a transmitter operating without a transmission 1069 limit, i.e. when $N_{r}=$ inf. As indicated in [74], optimizing 1070 the transmit power of the source was formulated as a convex 1071 optimization problem.

1072 Once the closed-form expression of (23) for the NEC $\bar{E}_{\text {total }}$ 1073 of a single hop, the optimized transmit power $P_{t_{1}}$ may be 1074 calculated by setting the derivative of (23) with respect to $P_{t_{1}}$ 1075 to zero, which yields

$$
\begin{aligned}
\frac{1}{p_{1}}+\frac{P_{t_{1}}}{p_{1}^{2}} \frac{d\left(1-p_{1}\right)}{d P_{t_{1}}} & =0 \\
\frac{p_{1}}{-P_{t_{1}}} & =\frac{d\left(1-p_{1}\right)}{d P_{t_{1}}},
\end{aligned}
$$

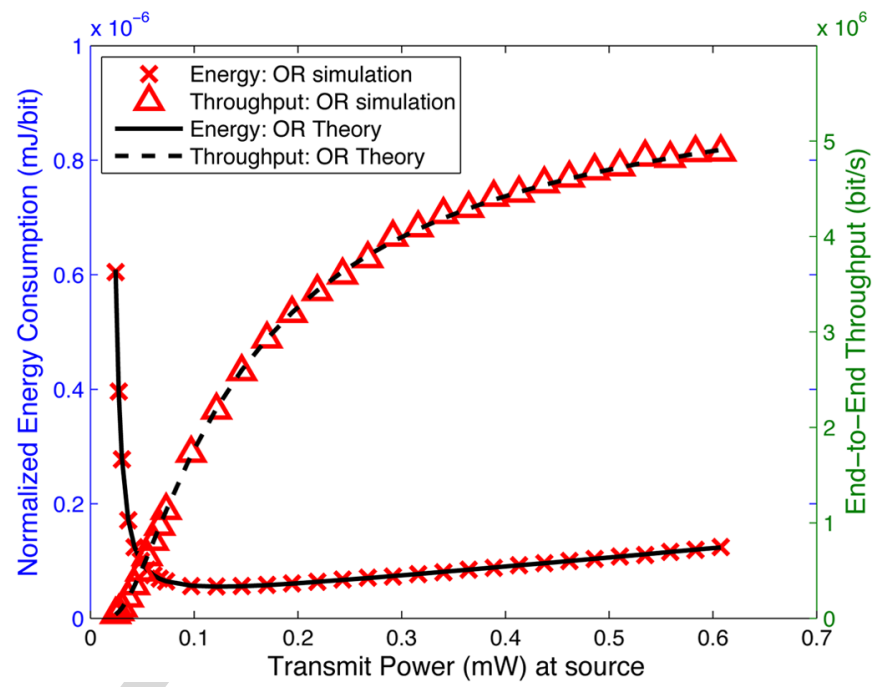

Fig. 23. The NEC $\bar{E}_{\text {total }}$ and the end-to-end throughput $R_{e 2 e}$ versus the transmit power $P_{t_{S}}$.

where $1-p_{1}=F E R_{1}$. Finally, the analytical expression of the 1076 optimized transmit power $P_{t_{1}}$ can be found. The existence of the 1077 optimized transmit power at the source of a single-hop route 1078 is shown in Fig. 23. Moreover, the end-to-end throughput $R_{e 2 e} 1079$ of the TR relying on an adjustable transmit power also obeys 1080 the same expression of (20). Therefore, the NEC $\bar{E}_{\text {total }}$ and the 1081 end-to-end throughput $R_{e 2 e}$ are compared both in terms of sim- 1082 ulation and theoretical results in Fig. 23, where the maximum 1083 number of MAC retransmissions is $N_{r}=7$. The frame length 1084 of the data packets, which are generated from the application 1085 layer, is 1024 Bytes. The $802.11 \mathrm{~g}$ standard is employed in the 1086 DL layer. The distance between $S$ and $D$ is $1000 \mathrm{~m}$. The other 1087 simulation configurations are listed in Table IV.

Fig. 23 shows that the NEC initially decreases and then 1089 increases slowly beyond the transmit power of $0.12 \mathrm{~mW}$, where 1090 $0.12 \mathrm{~mW}$ is the optimal transmit power obtained by using 1091 (24). The end-to-end throughput increases upon increasing the 1092 transmit power at $S$. Observe that the simulation results closely 1093 match the theoretical curve.

The idealized multi-hop linear network researched in 1095 Section III-A may be extended to a more realistic random net- 1096 work relying on Dijkstra's routing algorithm [124] and invoking 1097 the NEC $\bar{E}_{\text {total }}$ for route selection. Hence, a heuristic routing 1098 algorithm, namely the TR having an adjustable transmit 1099 power is invoked in [74] (referred to as Algorithm 1 in [74]), 1100 which may be adapted to the random network scenario 1101 considered for guaranteeing a high energy efficiency. For 1102 ease of interpretation, in this paper, the TR having an 1103 adjustable transmit power is exemplified with the aid of 1104 its step-by-step execution using the NEC metric $\bar{E}_{\text {total }}$, as 1105 shown in Fig. 24. It is assumed that $\mathcal{V}$ is the vertex set, $v$ is 1106 a node in the set $\mathcal{V}$ and $\bar{E}_{S v}$ denotes the NEC. Moreover, $S 1107$ represents the set of selected nodes, while $P_{t}^{o p t}(u, v)$ denotes 1108 the optimal transmit power of node $u$ assigned for transmission 1109 to node $v$.

As an example, the positions of $S, D, R_{1}$ and $R_{2}$ are as- 1111 sumed to be $(100,100),(900,100),(500,500)$, and $(300,1112$ 400), respectively. The IrCC-URC-QPSK is employed in the 1113 


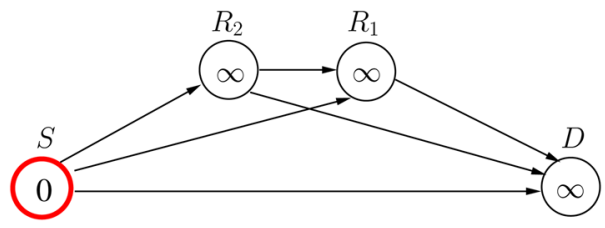

(a)

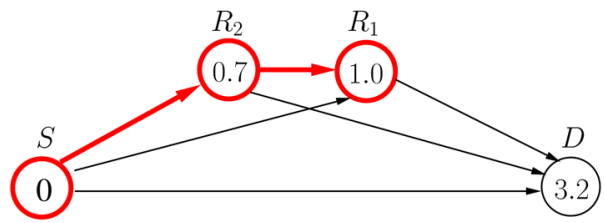

(c)

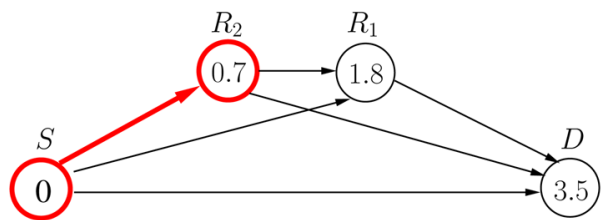

(b)

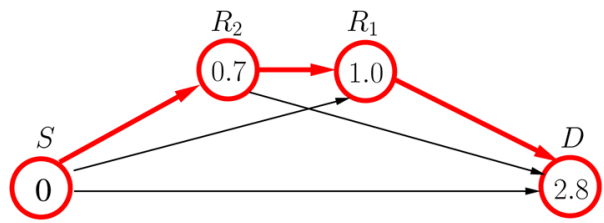

(d)

Fig. 24. Execution of the TR associated with an adjustable transmit power in a specific instance, where the positions of $S, D, R_{1}$ and $R_{2}$ are assumed to be $(100,100),(900,100),(500,500)$, and $(300,400)$, respectively. The value within a node $v$ is its energy cost $\bar{E}_{\text {total }}\left(\times 10^{-8} \mathrm{~mJ} / \mathrm{bit}\right)$ for transmission from $S$ to node $v$. After each iteration one node is incorporated into the set $S$. The nodes in boldface denote the nodes in $S$ after each iteration and the arrows in boldface represent the shortest route from $S$ to the nodes in boldface after each iteration. Due to the adjustable transmit power of node $u$, the probability $p_{f}(u, v)$ of a packet, which is dropped at any link $u-v$ after $N_{r}=7$ retransmissions, has nearly the same value of $p_{f}(u, v)=0.041$, hence this value next to the arrows is not plotted for simplicity. (a) The situation just after the initialization, where $S=\{S\}$. (b) The first iteration of the algorithm, where $S=\{S\}$ before the iteration, while after the iteration $R_{2}$ is incorporated into the set $S$ with the optimum power of $P_{t}^{o p t}\left(S, R_{2}\right)=0.16 \mathrm{~mW}$, yielding $S=\left\{S, R_{2}\right\}$. (c) The second iteration of the algorithm, where $S=\left\{S, R_{2}\right\}$ before the iteration, while after the iteration $R_{1}$ is incorporated into the set $S$ with the optimum power of $P_{t}^{o p t}\left(R_{2}, R_{1}\right)=0.06 \mathrm{~mW}$, yielding $\mathcal{S}=\left\{S, R_{2}, R_{1}\right\}$. (d) The final iteration of the algorithm, where $\mathcal{S}=\left\{S, R_{2}, R_{1}\right\}$ before the iteration, while after the iteration $D$ is incorporated into the set $S$ with the optimum power of $P_{t}^{o p t}\left(R_{1}, D\right)=0.39 \mathrm{~mW}$, yielding $S=\left\{S, R_{2}, R_{1}, D\right\}$. The algorithm terminates.

1114 PHY layer. The channel imposes both free-space path-loss and 1115 uncorrelated Rayleigh fading, plus the ubiquitous AWGN. The 1116 other relevant parameters are listed in Table IV. Each node is 1117 assumed to be aware of the other nodes' position, hence also 1118 of their distance. In a compact form, $\mathcal{V}=\left\{S, R_{1}, R_{2}, D\right\}$ and $1119 S=\{S\}$, as shown in Fig. 24(a). In Fig. 24(b), $S$ calculates its 1120 transmit power optimized for minimizing the NEC from (24), 1121 which is $\bar{E}_{S R_{1}}=1.8 \times 10^{-8} \mathrm{~mJ} / \mathrm{bit}, \bar{E}_{S R_{2}}=0.7 \times 10^{-8} \mathrm{~mJ} / \mathrm{bit}$, $1122 \bar{E}_{S D}=3.5 \times 10^{-8} \mathrm{~mJ} / \mathrm{bit}$ for transmission from $S$ to $R_{1}, R_{2}$ and $1123 \mathrm{D}$, respectively. Since $\bar{E}_{S R_{2}}=0.7 \times 10^{-8} \mathrm{~mJ} / \mathrm{bi}$ is the lowest 1124 in the set of the three energies, $S$ is updated to $\left\{S, R_{2}\right\}$. Then 1125 in Fig. 24(c), $R_{2}$ calculates its transmit power optimized for 1126 minimizing the NEC from (24) for the transmission, which 1127 is spanning from $S$ to node $R_{1}$ and $D$ via $R_{2}$, respectively. 1128 Since the updated NEC $\bar{E}_{S R_{1}}=1.0 \times 10^{-8} \mathrm{~mJ} / \mathrm{bit}$ is lower than $1129 \bar{E}_{S D}=3.2 \times 10^{-8} \mathrm{~mJ} / \mathrm{bit}, S$ is updated to $\left\{S, R_{2}, R_{1}\right\}$. Finally, in 1130 Fig. 24(d), $R_{1}$ adjusts its own transmit power to the optimal one, 1131 which minimizes the NEC $\bar{E}_{S D}=2.8 \times 10^{-8} \mathrm{~mJ} / \mathrm{bit}$ from $S$ to $1132 D$ via $R_{2}$ and $R_{1}$. At this stage, $D$ is incorporated into $S$. Since $1133 \mathcal{S}=\left\{S, R_{2}, R_{1}, D\right\}$, the TR with adjustable transmit power may 1134 be deemed to have converged and the route $S-R_{2}-R_{1}-D$ is 1135 deemed to be the optimal route for transmission from $S$ to $D$.

1136 The computational complexity has three main contributing 1137 factors: a) the calculation of a single NEC in a specific case; $1138 \mathrm{~b}$ ) the number of NEC calculations; c) and finally, finding the 1139 minimum NEC in each round. They denote the complexity of $1140 E_{s}, E_{f}$ and $p_{s}$, where $p_{s}=\prod_{1}^{H} B\left(p_{i}\right)$, by $C\left(E_{s}\right), C\left(E_{f}\right)$ and $1141 C\left(p_{s}\right)$. The complexity of evaluating $D_{s}$ and $D_{f}$ is the same 1142 as that of $E_{s}$ and $E_{f}$, apart from a multiplicative constant. 1143 The number of NEC calculations is given by the number of 1144 node pairs, which is $\mathcal{V}(\mathcal{V}-1) / 2$. The minimum NEC can be 1145 found based on the Fibonacci heap approach of [125], which 1146 has a complexity on the order of $\mathrm{O}(\log \mathcal{V})$. Therefore, the 1147 complexity imposed by the TR with adjustable transmit power 1148 is $\mathrm{O}\left[\mathcal{V}^{2}\left[\mathcal{C}\left(E_{s}\right)+C\left(E_{f}\right)+C\left(p_{s}\right)\right]+\mathcal{V} \log \mathcal{V}\right]$.
The performance of TR relying on an adjustable transmit 1149 power will be characterized in Section III-C in comparison to 1150 that of the OR of Section III-C.

\section{Opportunistic Routing With Adjustable Transmit Power}

The TR transmits the packet along the specific pre-selected 1153 route having the lowest estimated NEC. This pre-selected route 1154 is determined after the estimation and comparison of the NEC 1155 of each potential candidate route. The information invoked 1156 for routing decisions is gleaned during the process of route 1157 discovery, but this information may become stale owing to 1158 node-mobility. Instead, OR considers the potential chances of 1159 success for each candidate $\mathrm{RN}$, bearing in mind their time- 1160 variant channel conditions. Regardless of which particular RN 1161 receives the packet from the source successfully, if this RN has 1162 the highest priority in the forwarder RN list, it will forward the 1163 packet to the next RN. Naturally, the challenge in the design 1164 of the OR procedure is the beneficial selection of the forwarder 1165 RN set, the specific priority order of the potential forwarders 1166 and the avoidance of duplicate transmissions [110]. All the 1167 nodes in a node's neighbor list are assumed to belong to this 1168 node's forwarder R-list. The metric used for determining the 1169 priority order is the normalized energy required by this par- 1170 ticular RN for reaching D. Acknowledgement (ACK) packets 1171 are employed for avoiding the duplicate transmissions. The 1172 particular RN in the forwarder R-set, which has the highest 1173 priority owing to requiring the lowest energy will send the ACK 1174 first. The other RNs, which overhear the ACK will withdraw 1175 from the competition [126], [127].

A two-hop network is shown in Fig. 25, which has a sin- 1177 gle source $S$, a single destination $D$ and $M$ RNs $R_{1}, R_{2}, \cdots, 1178$ $R_{M-1}, R_{M} . S$ and $D$ are capable of communicating with all 1179 the RNs, as well as with each other. By contrast, the $M$ RNs 1180 are unable to communicate with each other. The idealized 1181 


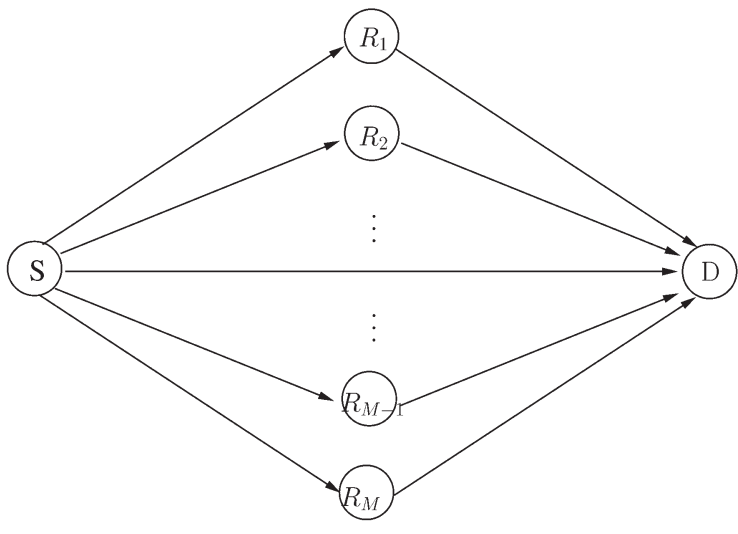

Fig. 25. A two-hop network assisted by a number of RNs.

1182 simplifying assumption is stipulated furthermore that each node 1183 knows the position of all other nodes. For each $\mathrm{RN} R_{m}, m=$ $11841 \ldots M$, the total average energy consumption $E_{R_{m} D}$ required 1185 for transmission from $R_{m}$ to $D$ is given by $E_{R_{m} D}=E_{R_{m} D}^{s}+E_{R_{m} D}^{f}$ 1186 where $E_{R_{m} D}^{s}$ represents the energy dissipated by a packet, which 1187 is successfully delivered from $R_{m}$ to $D$ while $E_{R_{m} D}^{f}$ represents 1188 the energy dissipated by a packet, which is dropped before 1189 reaching $D$ from $R_{m}$ after $N_{r}$ MAC retransmissions. Let $E_{S}$ 1190 denote the energy dissipated while sending a packet from 1191 the source $S$ to any of the RNs $R_{m}$, which is $E_{S}=P_{t_{S}} T$. It 1192 is assumed that $E_{R_{1} D}<E_{R_{2} D}<\cdots<E_{R_{M} D}$. Furthermore, for 1193 convenience, the destination node $D$ is represented as $R_{0}$ and $1194 \prod_{m=0}^{M}\left(1-p_{S R_{m}}\right)=\zeta$, where $p_{S R_{m}}$ denotes the probability of a 1195 packet, which is successfully delivered from $S$ to $R_{m}$.

1196 If $S$ successfully sends a packet to the $m$-th $\mathrm{RN}, m=$ $11970,1, \ldots, M$, with the aid of $n_{r}$ transmissions, the probability of 1198 this event is

$$
\begin{aligned}
& p_{0}\left(n_{r}\right)=\zeta^{n_{r}-1} p_{S R_{0}}, \text { if } m=0 \\
& p_{m}\left(n_{r}\right)=\zeta^{n_{r}-1} \prod_{i=0}^{m-1}\left(1-p_{S R_{i}}\right) p_{S R_{m}}, \text { if } 1 \leq m \leq M .
\end{aligned}
$$

1199 Correspondingly, the energy dissipated becomes

$$
\begin{aligned}
& E_{0}\left(n_{r}\right)=n_{r} E_{S}, \text { if } m=0 \\
& E_{m}\left(n_{r}\right)=n_{r} E_{S}+E_{R_{m} D}, \text { if } 1 \leq m \leq M
\end{aligned}
$$

1200 Let $D_{R_{m} D}$ denote the average delay of a packet travers1201 ing from $R_{m}, m=1, \ldots, M$, to $D$, including the delay $D_{R_{m} D}^{s}$ 1202 encountered by a packet that is successfully delivered to $D$ 1203 and the delay $D_{R_{m} D}^{f}$ experienced when a packet is dropped 1204 before reaching $D$. Then $D_{R_{m} D}=D_{R_{m} D}^{s}+D_{R_{m} D}^{f}$, where $D_{R_{m} D}^{s}$ 1205 represents $D_{s}$ and $D_{R_{m} D}^{f}$ corresponds to $D_{f}$, provided that the 1206 number of hops is 1 . Consequently,

$$
\begin{aligned}
D_{0}\left(n_{r}\right) & =n_{r} D_{S}, \text { if } m=0 \\
D_{m}\left(n_{r}\right) & =n_{r} D_{S}+D_{R_{m} D} \\
& =n_{r} D_{S}+\left(D_{R_{m} D}^{S}+D_{R_{m} D}^{f}\right) \text {, if } 1 \leq m \leq M,
\end{aligned}
$$

1207 where $D_{S}$ is $T$, which denotes the duration of a Time Slot (TS).
Consequently, when taking into account all the possible 1208 events, the total energy consumption is

$$
\begin{aligned}
E_{\text {total }}=\sum_{n_{r}=1}^{N_{r}} p_{0}\left(n_{r}\right) & E_{0}\left(n_{r}\right) \\
& +\sum_{n_{r}=1}^{N_{r}} \sum_{m=1}^{M} p_{m}\left(n_{r}\right) E_{m}\left(n_{r}\right)+\zeta^{N_{r}}\left(N_{r} E_{S}\right)
\end{aligned}
$$

while the total delay becomes:

$$
\begin{aligned}
D_{\text {total }}=\sum_{n_{r}=1}^{N_{r}} p_{0}\left(n_{r}\right) & D_{0}\left(n_{r}\right) \\
& +\sum_{n_{r}=1}^{N_{r}} \sum_{m=1}^{M} p_{m}\left(n_{r}\right) D_{m}\left(n_{r}\right)+\zeta^{N_{r}}\left(N_{r} D_{S}\right)
\end{aligned}
$$

Meanwhile, the packet transmitted from $S$ may be dropped 1211 in the $S-D, S-R_{m}$ or $R_{m}-D$ link, where $m=1, \ldots, M$ and 1212 again, the destination can be replaced by $R_{0}$. Then the end-to- 1213 end outage probability $p_{f}$ may be formulated as

$$
p_{f}=p_{f, S-R_{0}}+p_{f, S-R_{m}}+p_{f, R_{m}-D}, m=1, \ldots, M
$$

Furthermore, the NEC $\bar{E}_{\text {total }}$ may be formulated as

$$
\bar{E}_{\text {total }}=\frac{E_{\text {total }}}{1-p_{f}},
$$

while the end-to-end throughput $R_{e 2 e}$ is given by

$$
R_{e 2 e}=\frac{1-p_{f}}{D_{\text {total }}} .
$$

Although the network topology in Fig. 25 has only two hops, 1217 this algorithm may be extended to a large network, where 1218 the OR principle is employed for each hop. Meanwhile, the 1219 optimal transmit power of each node is found for the sake 1220 of minimizing the NEC required for the successful passage 1221 of a packet from that node to the destination. Therefore, the 1222 heuristic routing algorithm, namely the $\mathrm{OR}$ associated with 1223 an adjustable transmit power is conceived in [74] (referred 1224 to as Algorithm 2 in [74]), for calculating the minimum NEC 1225 by carrying out optimum distance-dependent power alloca- 1226 tion at each node, hop-by-hop. For ease of interpretation, in 1227 this paper, the OR having an adjustable transmit power is 1228 exemplified with the aid of its step-by-step execution using 1229 the NEC metric $\bar{E}_{\text {total }}$, as shown in Fig. 26. Here, for any 1230 node $v$ in a given vertex set $\mathcal{V}, \bar{E}_{v D}$ denotes the NEC $\bar{E}_{\text {total }} 1231$ necessitated for transmission from node $v$ to the destination 1232 $D$, where the potential set of receiver nodes is denoted by 1233 $\mathcal{R}$. Furthermore, $P_{t}^{o p t}(v)$ is the optimal transmit power, which 1234 minimizes the NEC required for transmission from node $v$ to 1235 the destination $D$.

Again, as a specific example, both the topology and the 1237 relevant parameters used in Fig. 26 are similar to those used 1238 in Fig. 24. It was also assumed that each node is aware of 1239 the other nodes' position, hence also of their distance. In a 1240 compact form, $\mathcal{V}=\left\{S, R_{1}, R_{2}, D\right\}$ and $\mathcal{R}=\{D\}$, as shown in 1241 


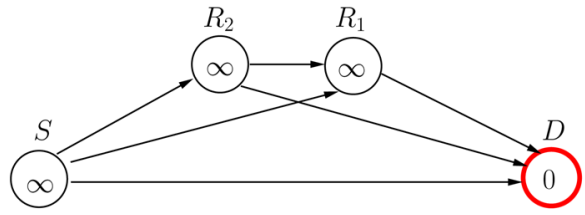

(a)

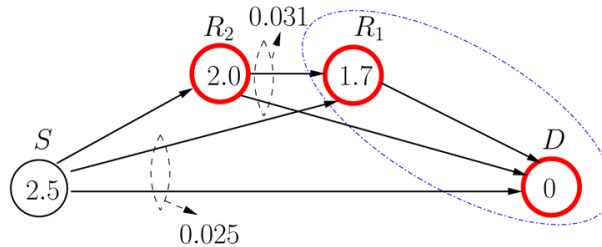

(c)

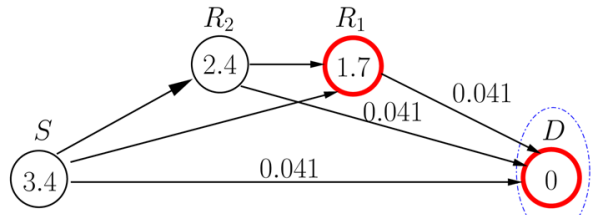

(b)

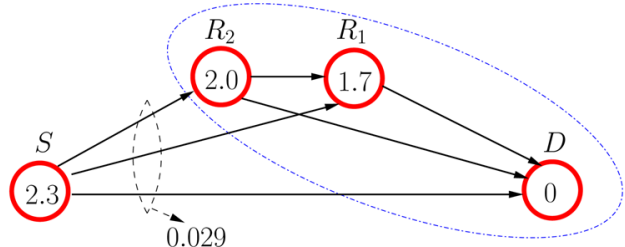

(d)

Fig. 26. Execution of the OR associated with an adjustable transmit power in a specific instance, where the positions of $S$, $D$, $R_{1}$ and $R_{2}$ are assumed to be $(100,100),(900,100),(500,500)$ and $(300,400)$, respectively. The value within a node $u$ is its cost $\bar{E}_{\text {total }}\left(\times 10^{-8} \mathrm{~mJ} / \mathrm{bit}\right)$ incurred by its transmission from node $u$ to $D$ and the dash-dot ellipse represents the receiver set $\mathcal{R}$ before each iteration. After each iteration one node is incorporated into the set $\mathcal{R}$. The nodes in boldface denote the nodes in $\mathcal{R}$ after each iteration. The values next to the arrows or the dashed ellipses represent the probability $p_{f}(u, \mathcal{R})$ of a packet being transmitted from $S$ in conjunction with the event that none of the nodes in the receiver set $\mathcal{R}$ receives it after $N_{r}=7$ retransmissions. (a) The situation just after the initialization, where $R=\{D\}$. (b) The first iteration of the algorithm, where $R=\{D\}$ before the iteration, while after the iteration $R_{1}$ is incorporated into the set $\mathcal{R}$ with the optimum power of $P_{t}^{\text {opt }}\left(R_{1}\right)=0.39 \mathrm{~mW}$, yielding $\mathcal{R}=\left\{R_{1}, D\right\}$. (c) The second iteration of the algorithm, where $\mathcal{R}=\left\{R_{1}, D\right\}$ before the iteration while after the iteration $R_{2}$ is incorporated into the set $\mathcal{R}$ with the optimum power of $P_{t}^{o p t}\left(R_{2}\right)=0.36 \mathrm{~mW}$, yielding $\mathcal{R}=\left\{R_{2}, R_{1}, D\right\}$. (d) The final iteration of algorithm, where $\mathcal{R}=\left\{R_{2}, R_{1}, D\right\}$ before the iteration, while after the iteration $S$ is incorporated into the set $\mathcal{R}$ with the optimum power of $P_{t}^{o p t}(S)=0.41$ mW, yielding $\mathcal{R}=\left\{S, R_{2}, R_{1}, D\right\}$. The algorithm terminates.

1242 Fig. 26(a). In Fig. 26(b), $S, R_{1}$ and $R_{2}$ calculate their transmit 1243 power optimized for minimizing the NEC from (34), yielding $1244 \bar{E}_{S D}=3.4 \times 10^{-8} \mathrm{~mJ} / \mathrm{bit}, \bar{E}_{R_{1} D}=1.7 \times 10^{-8} \mathrm{~mJ} / \mathrm{bit}, \bar{E}_{R_{2} D}=$ $12452.4 \times 10^{-8} \mathrm{~mJ} /$ bit for transmission to $D$. Since $\bar{E}_{R_{1} D}=1.7 \times$ $124610^{-8} \mathrm{~mJ} / \mathrm{bit}$ is the lowest in the set of the three energies, $R$ is 1247 updated to $\left\{R_{1}, D\right\}$. Then in Fig. 26(c), $S$ and $R_{2}$ adjust their 1248 own transmit power and update their NEC for transmission 1249 to node $D$ by considering $\left\{R_{1}, D\right\}$ as their forwarder relay 1250 set. Since $\bar{E}_{R_{2} D}=2.0 \times 10^{-8} \mathrm{~mJ} / \mathrm{bit}$ is lower than $\bar{E}_{S D}=$ $12512.5 \times 10^{-8} \mathrm{~mJ} / \mathrm{bit}, \mathcal{R}$ is updated to $\left\{R_{2}, R_{1}, D\right\}$. Finally, in 1252 Fig. 26(d), $S$ adjusts its own transmit power to the optimal one, 1253 which minimizes $\bar{E}_{S D}=2.3 \times 10^{-8} \mathrm{~mJ} /$ bit, where $\left\{R_{2}, R_{1}, D\right\}$ 1254 is the resultant forwarder relay set. At this stage, the OR with 1255 adjustable transmit power may be deemed to have converged, 1256 since $S$ is incorporated into $\mathcal{R}$ and $\mathcal{R}=\left\{S, R_{2}, R_{1}, D\right\}$. In this 1257 algorithm, every node has to find its own forwarder $\mathrm{R}$-set 1258 by itself upon exploiting the knowledge of the other nodes' 1259 positions. If more than one node in a node's forwarder R1260 list receives the packet from that node successfully, then that 1261 particular one, which requires the lowest NEC for transmission 1262 to the destination has the highest priority for forwarding this 1263 packet. The nodes of the forwarder R-set communicate with 1264 each other similarly to the technique of [126] and again, the 1265 NEC required for successful transmission to $D$ is invoked for 1266 deciding the priority order of the forwarders.

1267 The complexity of finding the transmit power and the for1268 warder set also depends on three contributing factors, just like 1269 for the TR scenario. They denote the complexity of $E_{\text {total }}$ in 1270 (34) and of $p_{f}$ in (33) by $C\left(E_{\text {total }}\right)$ and $C\left(p_{f}\right)$, respectively. 1271 The OR with adjustable transmit power has to invoke $\mathcal{V}$ times 1272 for the sake of adding a further node into $R$ in each round. The 1273 optimal transmit power of any node in $(\mathcal{V}-\mathcal{R})$ is calculated 1274 in a specific round and the complexity of this calculation is 1275 given by $C\left(E_{\text {total }}\right)+C\left(p_{f}\right)$. Again, the complexity of finding 1276 the optimal transmit power can be calculated by Fibonacci heap [125] which has a complexity on the order of $\mathrm{O}(\log \mathcal{V}) .1277$ Therefore, the complexity of the OR with adjustable transmit 1278 power is $\mathrm{O}\left[\mathcal{V}^{2}\left[C\left(E_{\text {total }}\right)+C\left(p_{f}\right)\right]+V \log \mathcal{V}\right]$.

Now the performance of the networks associated with a total 1280 of $N=4$ and 15 nodes are analyzed. The positions of $S$ and 1281 $D$ are $(100,100)$ and $(900,100)$, respectively, while the other 1282 nodes are uniformly located within a circle centered at $(400,1283$ $100)$ with a radius of $400 \mathrm{~m}$. The NEC $\bar{E}_{\text {total }}$ and the end- 1284 to-end throughput $R_{e 2 e}$ are shown in Fig. 27 and Fig. 28 as 1285 a function of the maximum number of MAC retransmissions 1286 $N_{r}$. The theoretical NEC bound of both TR and OR was also 1287 invesgated when $N=4$, which was found by the exhaustive 1288 search of all the routes spanning from $S$ to $D$, while for $N=151289$ no theoretical bounds were given, since the exhaustive search 1290 has an excessive computation complexity.

1291

Fig. 27 shows that the performance of the energy- 1292 consumption OF based algorithm is close to the theoretical 1293 bound when $N=4$, especially in the case of a high $N_{r}$. Both 1294 Figs. 27 and 28 show that the energy-efficient OR outperforms 1295 both the Adjustable Energy-Efficient Opportunistic Routing 1296 (A-EEOR) algorithm defined in [91] and the energy-efficient 1297 TR. Here, the A-EEOR algorithm selects and prioritizes the 1298 forwarding set during the initialization stage according to the 1299 total energy cost of forwarding a packet to the destination node, 1300 which is estimated under the assumption of allowing a poten- 1301 tially infinite number of MAC retransmissions $N_{r}$. However, 1302 $N_{r}$ is finite in practical scenarios. Hence, more specifically, 1303 compared to the A-EEOR algorithm the OR algorithm has 1304 a lower normalized energy consumption for $N_{r}<4$, as seen 1305 in Fig. 27, while exhibiting a higher end-to-end throughput 1306 for $N_{r}<6$, as shown in Fig. 28. Moreover, both the OR and 1307 TR simulation results closely match the theoretical curves. 1308 When $N_{r}=1$ or 2 for the network topology of $N=4$, both 1309 the exhaustive search, labelled by "TR bound" and the TR 1310 algorithm proposed in [74], labelled by "TR theory", selected 1311 


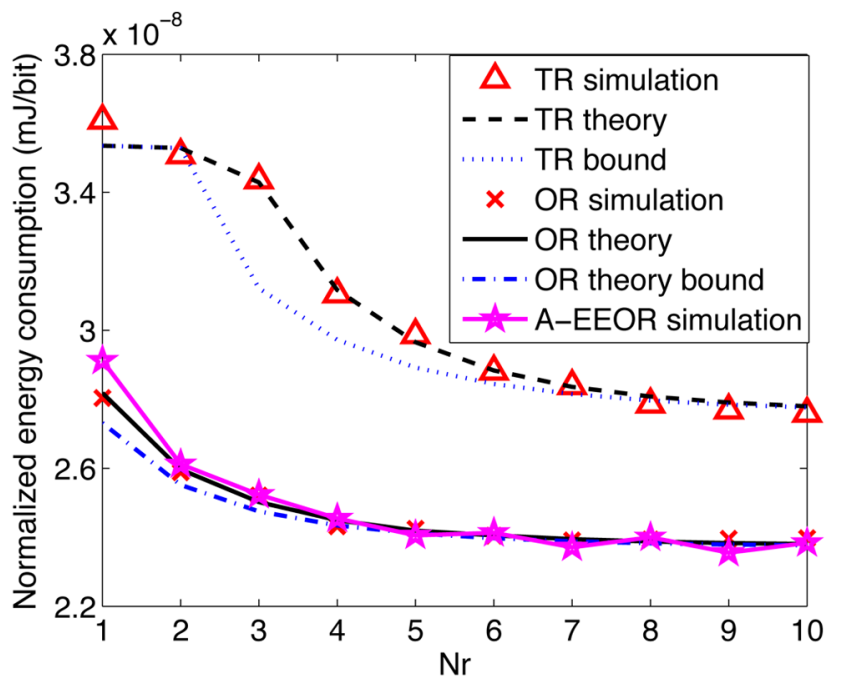

(a)

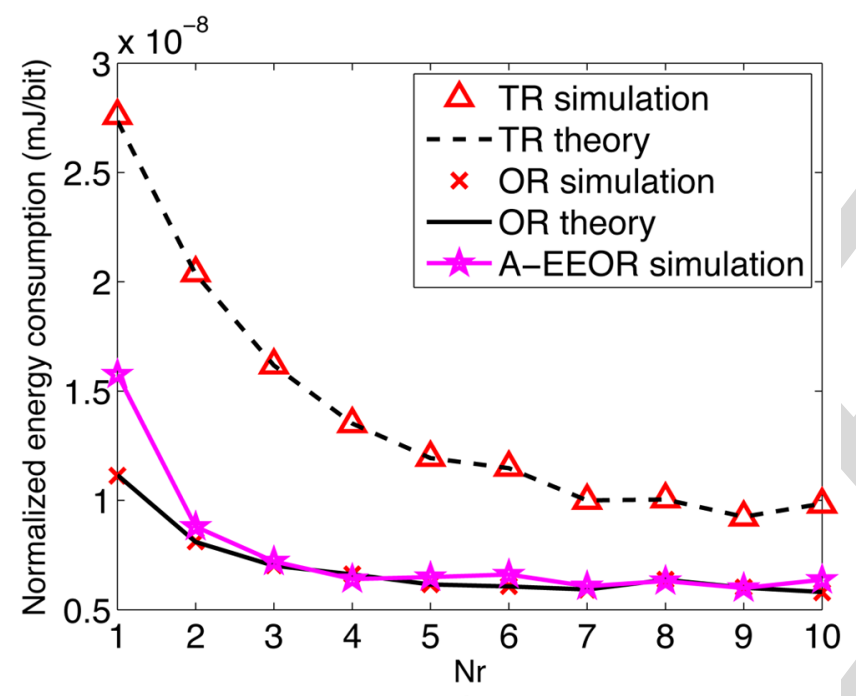

(b)

Fig. 27. The NEC $\bar{E}_{\text {total }}$ versus the maximum number of MAC retransmissions $N_{r}$ when $N=4$ and 15. (a) Network topology $N=4$. (b) Network topology $N=15$.

1312 the route 'S-D'. Hence the NEC is the same for both. When $13132<N_{r}<8$, the exhaustive search and the TR algorithm 1314 proposed in [74] choose different routes, since the exhaustive 1315 search represents the globally optimal algorithm, while the TR 1316 algorithm is a locally optimal algorithm. More specifically, the 1317 TR algorithm is optimal for every single hop. Moreover, the 1318 simulation results corresponding to the 'TR simulation' label 1319 closely match the theoretical value represented by the label 1320 'TR theory'. Therefore, the 'TR simulation/theory' and the 'TR 1321 bound' scenarios exhibit a performance discrepancy, when $2<$ $1322 N_{r}<8$, as seen in Fig. 27. Note that the NEC $\bar{E}_{\text {total }}$ decreases 1323 upon increasing $N_{r}$. However, the end-to-end throughput $R_{e 2 e}$ of 1324 the A-EEOR and OR regimes first increases and then saturates. 1325 Additionally, the end-to-end throughput of TR is in fact higher 1326 than that of OR for $N_{r}=1$ and 2 when $N=4$, but it is lower 1327 for $N_{r} \geq 3$, as seen in Fig. 28. This is because in case of a low 1328 number of MAC retransmissions, the direct near-line-of-sight 1329 route spanning from $S$ to $D$ in the TR has a more dominant 1330 priority than the other routes.

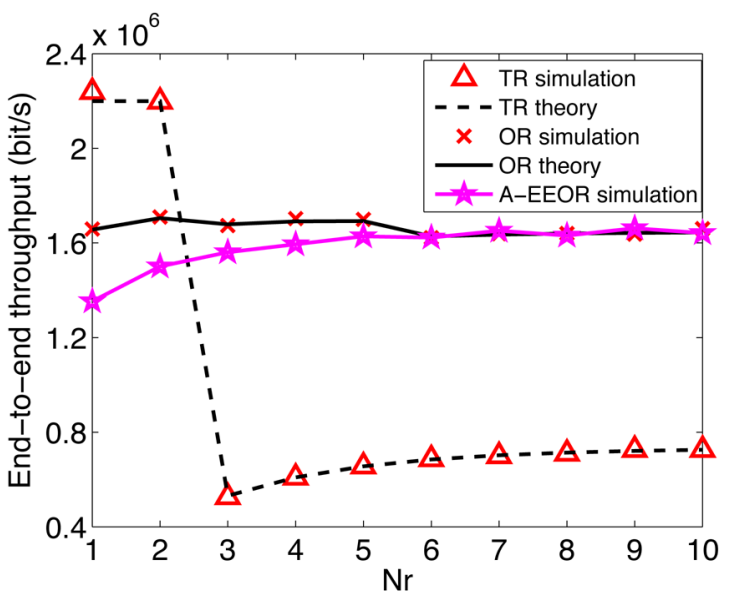

(a)

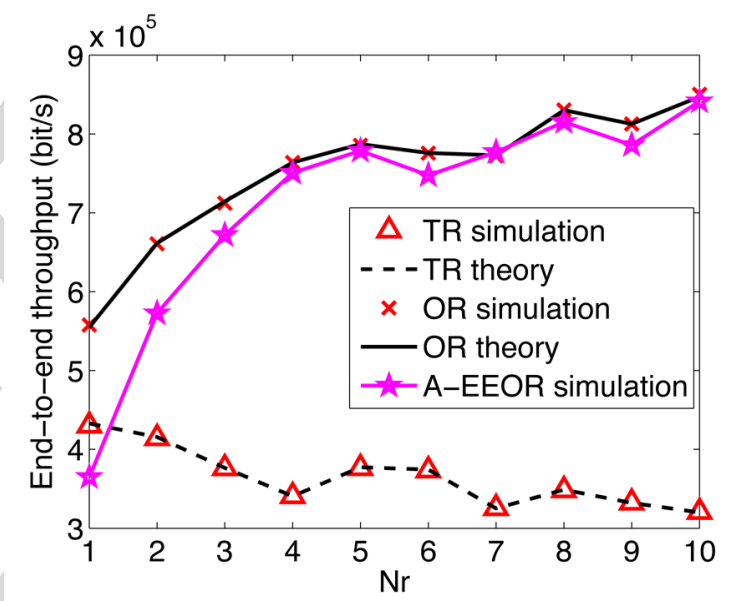

(b)

Fig. 28. The end-to-end throughput $R_{e 2 e}$ versus the maximum number of MAC retransmissions $N_{r}$ when $N=4$ and 15. (a) Network topology $N=4$. (b) Network topology $N=15$.

\section{Conclusions And Design Guidelines}

\section{A. Conclusions}

In this paper diverse routing schemes were studied, investi- 1333 gating the benefits of multi-antenna aided RNs, the FER, the 1334 number of MAC retransmissions and the number of hops on 1335 the performance energy consumption.

- In Section I, we described the main functions of the OSI 1337 model layer by layer, then we highlighted the common 1338 methods of cross-layer design. The historical develop- 1339 ment of cross-layer aided routing protocol designs was 1340 portrayed in Table II. Then, we categorized the family 1341 of ad hoc routing protocols, which were improved in the 1342 following chapters.

- In Section II, we focused our attention on the reduction 1344 of the energy consumption by exploiting the benefits of 1345 the coordination between the PHY layer and the NET 1346 layer. Specifically, the advantages of near-capacity coding 1347 schemes were quantified in terms of their energy saving. A 1348 near-capacity three-stage concatenated IrCC-URC-STTC 1349 relay-transceiver equipped with two transmit antennas was 1350 proposed in [72] for the ad hoc network considered, 1351 since it achieved a low FER at a low transmit power. 1352 


\section{B. Design Guidelines}

1368 In general, three basic steps may be identified, when design1369 ing routing algorithms in ad hoc networks, which are:

The high effective transmission range of the IrCC-URCSTTC aided MA-RNs facilitated cross-layer design for activating beneficial routes having the lowest number of longer hops.

- Section III was specifically dedicated to minimizing the energy consumed by the data packets during the process of data transmission, where the NEC was quantified by considering both the PHY layer as well as the DL layer and the NET layer. Additionally, a cross-layer operation aided energy-efficient OR algorithm for ad hoc networks and an energy-consumption-based OF combined with PA was analyzed, which was proposed in [74] both for finding a theoretical bound and for conveying the packets through the network.

1) Determining the design targets, such as the network's throughput and/or energy consumption;

2) Determining the key factors, which influence the design targets most crucially. These key factors may be related to different layers, including the channel categories, the protocol parameters and so on;

3) Determining the routing metrics used for making routing decisions, such as the number of hops and/or the normalized end-to-end energy consumption.

Let us now detail these three design steps as follows:

- Throughput and energy consumption constitute a pair of important specifications in analyzing a network's performance, which critically depend on the parameters of the different OSI layers. Hence, combining the functions of multiple layers with the aid of cross-layer operation is beneficial in terms of improving the attainable performance, as demonstrated in this tutorial with the assistance of several cross-layer aided routing algorithms designed for $a d$ hoc networks.

- The number of hops is one of the most popular routing metrics in routing design, as we demonstrated in the context of the classic routing algorithm, namely the DYMO protocol.

- One of the most important factors we have to consider in the PHY layer is constituted by the specific characteristics of the time-variant wireless channel, which inflict bit/symbol errors and even packet loss events at the receiver node. Hence, strong and robust channel coding schemes have to be employed for mitigating the channelinduced degradations. The BER and FER are the two representative parameters, which are capable of characterizing the influence of both the time-variant wireless channel and of the FEC coding schemes, hence representing the overall performance of the PHY layer.

- For the sake of reducing the system's total transmit energy consumption, a near-capacity coding scheme, such as the IrCC-URC-STTC scheme of Section II-A is the most appropriate choice, since it requires a reduced transmit power at a given BER/FER value, which may also be 1408 viewed as reducing the BER/FER at a given transmit 1409 power. This is the reason, why the IrCC-URC-STTC aided 1410 MA transceivers operate close to the achievable capacity 1411 and this is, why they are capable of reducing the num- 1412 ber of hops spanning from the source to the destination. 1413 Requiring less hops implies that less nodes are involved, 1414 hence reducing the energy dissipation. An energy-efficient 1415 routing algorithm relying on the employment of IrCC- 1416 URC-STTC aided MA transceivers [72] was analyzed in 1417 Section II-B and Section II-C, showing that the system's 1418 total transmit energy consumption was reduced.

1419

- Furthermore, having considered the factors influencing the 1420 design of both the PHY layer and of the NET layer, we 1421 have to proceed by characterizing the influence of the DL 1422 layer in the cross-layer aided routing design. Our goal 1423 is that of achieving a throughput improvement and for 1424 energy reduction. One of the representative factors in the 1425 DL layer is constituted by the number of maximum MAC 1426 retransmissions. The larger the number of maximum MAC 1427 retransmissions, the more energy will be consumed and 1428 the higher the delay becomes. As a benefit, the success- 1429 ful packet reception probability is improved. Hence, we 1430 have to find the most appropriate number of maximum 1431 MAC retransmissions for the sake of striking an attractive 1432 compromise.

1433

- Additionally, we emphasize that the energy assigned to 1434 the data packets plays a dominant role in determining the 1435 system's total energy dissipation, which hence has to be 1436 optimized. For the sake of achieving an improved network 1437 throughput and a reduced energy consumption, the joint 1438 influence of the FER in the PHY layer, of the maximum 1439 number of retransmissions in the DL layer and of the 1440 number of hops in the NET layer has to be carefully con- 1441 sidered. Additionally, opting for the NEC as the routing 1442 metric instead of the number of hops is more beneficial in 1443 terms of reducing energy consumption. Hence, an accurate 1444 energy-consumption-based $\mathrm{OF}$ is required for combining 1445 all the three factors corresponding to the lower three 1446 layers of the seven-layer OSI architecture, as indicated 1447 in Section III-A of the tutorial. The routing algorithm 1448 proposed strikes an attractive tradeoff between the normal- 1449 ized energy consumption and the end-to-end throughput 1450 in the context of real-world scenarios, as exemplified in 1451 Section III-A.

- A hop-length-dependent PA is beneficial in terms of re- 1453 ducing the energy consumption. If the transmit power of 1454 each node is assumed to be the same, a certain amount of 1455 extra energy will be dissipated, since the distance between 1456 each pair of nodes is different, which would necessitate a 1457 different amount of transmit energy. An energy-efficient 1458 TR algorithm was designed with the aid of the hop-length- 1459 dependent power allocation of Section III-B, which also 1460 jointly considered the FER in the PHY layer, the maximum 1461 number of retransmissions in the DL layer and the number 1462 of hops in the NET layer. A reliable routing metric is 1463 constituted by the NEC quantified in terms of the energy- 1464 based OF exemplified in Section III-B. 
1477 All operational systems rely on a vital form of cross1478 layer operation, which makes wireless systems different 1479 from wireline based systems. Explicitly, both handovers and 1480 power-control rely on cross-layer cooperation in all wireless 1481 systems. This is why they are usually shown diagrammati1482 cally as a block bridging the lowest three layers. Going back 1483 as far as the old second-generation GSM system, the total 1484 control-channel bitrate was as low as $961 \mathrm{bits} / \mathrm{sec}$, which 1485 limited the efficiency of the power-control and handover 1486 operations, especially at high velocity and for small traffic 1487 cells, when handovers are frequent. For the 3G systems the 1488 control-channel rates were increased by an order of mag1489 nitude to about $10 \mathrm{kbits} / \mathrm{s}$, which facilitated more prompt 1490 handovers and power-control actions, when for example 1491 the mobile turned at a street-corner. The 4G LTE system 1492 also followed this trend, since an increased control-channel 1493 rate supports more sophisticated cross-layer cooperation. 1494 Although the main focus of this tutorial is on the energy dis1495 sipated by data packets during the process of data transmission, 1496 we note that cross-layer cooperation imposes an extra network 1497 overhead, since the control information also plays an impor1498 tant role in determining the system's total energy consumption, 1499 especially in mobile scenarios where the control information 1500 assists in maintaining seamless communications [128]. The 1501 extra control information is generated, when the informa1502 tion exchange takes place amongst layers or different nodes, 1503 including the control bits and the extra control packets. 1504 Additionally, a plethora of control packets are required for both 1505 route discovery and for route maintenance. For example, node1506 mobility might cause the following problems:

1507

1508

1509

1510

1511

1512

1513

1514

1515

1516 Hence, the energy-consumption-based OFs formulated in the 1517 stationary scenario may require further adjustments, if the en1518 ergy dissipated by the control packets is also considered. It may 1519 be promising to employ bio-inspired algorithms, such as the 1520 ant colony algorithm [132], for accommodating a dynamically 1521 changing topology, which requires future research.
REFERENCES

[1] H. Labiod, Ed., Wireless Ad Hoc and Sensor Networks. Hoboken, NJ, 1523 USA: Wiley, 2008.

[2] R. Ramanathan and J. Redi, "A brief overview of Ad Hoc networks: 1525 Challenges and directions," IEEE Commun. Mag., vol. 40, no. 5, pp. 20- 1526 22, May 2002.

[3] H. Zimmermann, "OSI reference model-the ISO model of architecture 1528 for open systems interconnection," IEEE Trans. Commun., vol. 28, no. 4, 1529 pp. 425-432, Apr. 1980.

[4] Reference Model of Open Systems Interconnection, ISO/TC97/SC16 Std. 1531 Doc. N227, 1979.

[5] A. Goldsmith, Wireless Communications. New York, NY, USA: 1533 Cambridge Univ. Press, 2005.

[6] W. Stallings, Wireless Communications \& Networks, 2nd ed. 1535 Englewood Cliffs, NJ, USA: Prentice-Hall, 2005.

[7] Information Technology-Telecommunications and Information Exchange 1537 Between Systems-Local and Metropolitan Area Networks-Specific 1538 Requirements, IEEE Std. 802.11, 2007.

[8] "Internet protocol," IETF Draft, 1981.

[9] S. Deering and R. Hinden, "Internet protocol, version 6 (IPv6) specifica- 1541 tion," IETF Draft, 1998

[10] "Transmission control protocol," IETF Draft, 1981

[11] “User datagram protocol," IETF Draft, 1980.

[12] Definitions of Terms Related to Quality of Service, ITU-T E.800, 2008. 1545

[13] V. Srivastava and M. Motani, "Cross-layer design: A survey and the road 1546 ahead," IEEE Commun. Mag., vol. 43, no. 12, pp. 112-119, Dec. 2005. 1547

[14] M. Conti, G. Maselli, G. Turi, and S. Giordano, "Cross-layering in 1548 mobile Ad Hoc network design," Computer, vol. 37, no. 2, pp. 48-51, 1549 Feb. 2004.

[15] R. Jurdak, Wireless Ad Hoc and Sensor Networks: A Cross-layer Design 1551 Perspective. New York, NY, USA: Springer-Verlag, 2010.

[16] F. Fu and M. V. D. Schaar, "A new systematic framework for autonomous 1553 cross-layer optimization," IEEE Trans. Veh. Technol., vol. 58, no. 4, 1554 pp. 1887-1903, May 2009.

[17] R. Winter, J. H. Schiller, N. Nikaein, and C. Bonnet, "Crosstalk: Cross- 1556 layer decision support based on global knowledge," IEEE Commun. 1557 Mag., vol. 44, no. 1, pp. 93-99, Jan. 2006.

[18] E. Setton, T. Yoo, X. Zhu, A. Goldsmith, and B. Girod, "Crosslayer de- 1559 sign of Ad Hoc networks for real-time video streaming," IEEE Wireless 1560 Commun., vol. 12, no. 4, pp. 59-65, Aug. 2005.

[19] Q. Liu, X. Wang, and G. B. Giannakis, "A cross-layer scheduling al- 1562 gorithm with QoS support in wireless networks," IEEE Trans. Veh. 1563 Technol., vol. 55, no. 3, pp. 839-847, May 2006.

[20] W. L. Huang and K. B. Letaief, "Cross-layer scheduling and power con- 1565 trol combined with adaptive modulation for wireless Ad Hoc networks," 1566 IEEE Trans. Commun., vol. 55, no. 4, pp. 728-739, Apr. 2007.

[21] Q. Zhang and Y.-Q. Zhang, "Cross-layer design for QoS support in 1568 multihop wireless networks," Proc. IEEE, vol. 96, no. 1, pp. 64-76, 1569 Jan. 2008.

[22] B. J. Oh and C. W. Chen, "A cross-layer approach to multichannel MAC 1571 protocol design for video streaming over wireless Ad Hoc networks," 1572 IEEE Trans. Multimedia, vol. 11, no. 6, pp. 1052-1061, Oct. 2009.

[23] S. Chu and X. Wang, "Opportunistic and cooperative spatial multiplex- 1574 ing in MIMO Ad Hoc networks," IEEE/ACM Trans. Netw., vol. 18, no. 5, 1575 pp. 1610-1623, Oct. 2010.

[24] A. Ghosh and W. Hamouda, "Cross-layer antenna selection and channel 1577 allocation for MIMO cognitive radios," IEEE Trans. Wireless Commun., 1578 vol. 10, no. 11, pp. 3666-3674, Nov. 2011.

[25] M. Mardani, S.-J. Kim, and G. B. Giannakis, "Cross-layer design 1580 of wireless multihop random access networks," IEEE Trans. Signal 1581 Process., vol. 60, no. 5, pp. 2562-2574, May 2012.

[26] M. Uddin, C. Rosenberg, W. Zhuang, P. Mitran, and A. Girard, "Joint 1583 routing and medium access control in fixed random access wireless 1584 multihop networks," IEEE/ACM Trans. Netw., vol. 22, no. 1, pp. 80-93, 1585 Feb. 2014

[27] F. Tang, L. Barolli, and J. Li, "A joint design for distributed stable 1587 routing and channel assignment over multihop and multiflow mobile 1588 Ad Hoc cognitive networks," IEEE Trans. Ind. Informat., vol. 10, no. 2, 1589 pp. 1606-1615, May 2014.

[28] V. Kawadia and P. R. Kumar, "A cautionary perspective on cross-layer 1591 design," IEEE Wireless Commun., vol. 12, no. 1, pp. 3-11, Feb. 2005.

[29] E. M. Royer and C.-K. Toh, "A review of current routing protocols for 1593 Ad Hoc mobile wireless networks," IEEE Pers. Commun., vol. 6, no. 2, 1594 pp. 46-55, Apr. 1999.

[30] C. E. Perkins and P. Bhagwat, "Highly dynamic destination-sequenced 1596 distance vector (DSDV) for mobile computers," in Proc. ACM SIGCOMM, 1597 Aug. 31-Sep. 2, 1994, pp. 234-244. 
31] T. Clausen and P. Jacquet, Optimized Link State Routing Protocol (OLSR) (RFC 3626) 2003, IETF Draft.

[32] The Dynamic Source Routing Protocol (DSR) for Mobile Ad Hoc Networks for IPv4. [Online]. Available: http://tools.ietf.org/html/rfc 4728

[33] Ad hoc On-Demand Distance Vector (AODV) Routing. [Online]. Available: http://tools.ietf.org/html/rfc3561

[34] Dynamic MANET On-Demand (DYMO) Routing Routing. [Online]. Available: http://tools.ietf.org/html/draft-ietf-manet-dymo-19

[35] The Zone Routing Protocol (ZRP) for Ad Hoc Networks. [Online]. Available: http://tools.ietf.org/html/draft-ietf-manet-zone-zrp-04

[36] A. Goldsmith and S. B. Wicker, "Design challenges for energyconstrained Ad Hoc wireless networks," IEEE Wireless Commun., vol. 9, no. 4, pp. 8-27, Aug. 2002.

[37] M. R. Souryal, B. R. Vojcic, and R. L. Pickholtz, "Information efficiency of multihop packet radio networks with channel-adaptive routing," IEEE J. Sel. Areas Commun., vol. 23, no. 1, pp. 40-50, Jan. 2005.

[38] S.-H. Lee, E. Choi, and D.-H. Cho, "Timer-based broadcasting for power-aware routing in power-controlled wireless Ad Hoc networks," IEEE Commun. Lett., vol. 9, no. 3, pp. 222-224, Mar. 2005.

[39] M. Johansson and L. Xiao, "Cross-layer optimization of wireless networks using nonlinear column generation," IEEE Trans. Wireless Commun., vol. 5, no. 2, pp. 435-445, Feb. 2006.

[40] S. Mao et al., "On routing for multiple description video over wireless Ad Hoc networks," IEEE Trans. Multimedia, vol. 8, no. 5, pp. 10631074 , Oct. 2006

[41] A. Abdrabou and W. H. Zhuang, "A position-based QoS routing scheme for UWB mobile Ad Hoc networks," IEEE J. Sel. Areas Commun., vol. 24, no. 4, pp. 850-856, Apr. 2006.

[42] J. Zhang, Q. Zhang, B. Li, X. Luo, and W. Zhu, "Energy-efficient routing in mobile Ad Hoc networks: Mobility-assisted case," IEEE Trans. Veh. Technol., vol. 55, no. 1, pp. 369-379, Jan. 2006.

[43] S. Kompella, S. Mao, Y. T. Hou, and H. D. Sherali, "Cross-layer optimized multipath routing for video communications in wireless networks," IEEE J. Sel. Areas Commun., vol. 25, no. 4, pp. 831-840, May 2007.

[44] M. Chiang, S. H. Low, A. R. Calderbank, and J. C. Doyle, "Layering as optimization decomposition: A mathematical theory of network architectures," Proc. IEEE, vol. 95, no. 1, pp. 255-312, Jan. 2007.

[45] K. T. Phan, H. Jiang, C. Tellambura, S. A. Vorobyov, and R. Fan, "Joint medium access control, routing and energy distribution in multihop wireless networks," IEEE Trans. Wireless Commun., vol. 7, no. 12, pp. 5244-5249, Dec. 2008.

[46] J. Liu, Y. T. Hou, Y. Shi, and H. D. Sherali, "Cross-layer optimization for MIMO-based wireless Ad Hoc networks: Routing, power allocation, bandwidth allocation," IEEE J. Sel. Areas Commun., vol. 26, no. 6, pp. 913-926, Aug. 2008.

[47] A. Abdrabou and W. H. Zhuang, "Statistical QoS routing for IEEE 802.11 multihop Ad Hoc networks," IEEE Trans. Wireless Commun., vol. 8, no. 3, pp. 1542-1552, Mar. 2009.

[48] P. Li, Q. Shen, Y. Fang, and H. Zhang, "Power controlled network protocols for multi-rate Ad Hoc networks," IEEE Trans. Wireless Commun., vol. 8, no. 4, pp. 2142-2149, Apr. 2009.

[49] L. Ding, T. Melodia, S. N. Batalama, J. D. Matyjas, and M. J. Medley, "Cross-layer routing and dynamic spectrum allocation in cognitive radio Ad Hoc networks," IEEE Trans. Veh. Technol., vol. 59, no. 4, pp. 19691979, May 2010.

[50] Y. Lu, J. Guan, Z. Wei, and Q. Wu, "Joint channel assignment and crosslayer routing protocol for multi-radio multi-channel Ad Hoc networks," J. Syst. Eng. Electron., vol. 21, no. 6, pp. 1095-1102, Dec. 2010.

[51] Z. Ding and K. K. Leung, "Cross-layer routing using cooperative transmission in vehicular Ad-Hoc networks," IEEE Journal on Selected Areas in Communications, vol. 29, no. 3, pp. 571-581, Mar. 2011.

[52] B. Tavli and W. B. Heinzelman, "Energy-efficient real-time multicast routing in mobile Ad Hoc networks," IEEE Trans. Comput., vol. 60, no. 5, pp. 707-722, May 2011.

[53] S.-J. Syue, C.-L. Wang, T. Aguilar, V. Gauthier, and H. Afifi, "Cooperative geographic routing with radio coverage extension for SER constrained wireless relay networks," IEEE J. Sel. Areas Commun., vol. 30, no. 2, pp. 271-279, Feb. 2012.

[54] M. Pan, H. Yue, C. Zhang, and Y. Fang, "Path selection under budget constraints in multihop cognitive radio networks," IEEE Trans. Mobile Comput., vol. 12, no. 6, pp. 1133-1145, Jun. 2013.

[55] J. G. Li, D. Cordes, and J. Y. Zhang, "Power-aware routing protocols in Ad Hoc wireless networks," IEEE Wireless Commun., vol. 12, no. 6, pp. 69-81, Dec. 2005.

[56] S. D. Muruganathan, D. C. F. Ma, R. I. Bhasin, and A. Fapojuwo, "A centralized energy-efficient routing protocol for wireless sensor networks," IEEE Commun. Mag., vol. 43, no. 3, pp. S8-S13, Mar. 2005.
[57] J. Zhu, C. Qiao, and X. Wang, "On accurate energy consumption models 1677 for wireless Ad Hoc networks," IEEE Trans. Wireless Commun., vol. 5, 1678 no. 11, pp. 3077-3086, Nov. 2006.

1679 AQ2

[58] S. J. Baek and G. Veciana, "Spatial energy balancing through proactive 1680 multipath routing in wireless multihop networks," IEEE/ACM Trans. 1681 Netw., vol. 15, no. 1, pp. 93-104, Feb. 2007.

[59] S. Eidenbenz, G. Resta, and P. Santi, "The COMMIT protocol for 1683 truthful and cost-efficient routing in Ad Hoc networks with self- 1684 ish nodes," IEEE Trans. Mobile Comput., vol. 7, no. 1, pp. 19-33, 1685 Jan. 2008.

1686

[60] W. Liang, R. Brent, Y. Xu, and Q. Wang, "Minimum-energy all- 1687 toall multicasting in wireless Ad Hoc networks," IEEE Trans. Wireless 1688 Commun., vol. 8, no. 11, pp. 5490-5499, Nov. 2009.

1689

[61] M. Li, L. Ding, Y. Shao, Z. Zhang, and B. Li, "On reducing broadcast 1690 transmission cost and redundancy in Ad Hoc wireless networks using di- 1691 rectional antennas," IEEE Trans. Veh. Technol., vol. 59, no. 3, pp. 1433- 1692 1442, Mar. 2010.

1693

[62] C. Ma and Y. Yang, "A battery-aware scheme for routing in wireless 1694 Ad Hoc networks," IEEE Trans. Veh. Technol., vol. 60, no. 8, pp. 3919- 1695 3932, Oct. 2011.

[63] A. M. Akhtar, M. R. Nakhai, and A. H. Aghvami, "Power aware cooper- 1697 ative routing in wireless mesh networks," IEEE Commun. Lett., vol. 16, 1698 no. 5, pp. 670-673, May 2012.

1699

[64] T. Lu and J. Zhu, "Genetic algorithm for energy-efficient QoS multicast 1700 routing," IEEE Commun. Lett., vol. 17, no. 1, pp. 31-34, Jan. 2013.

[65] J. Vazifehdan, R. Prasad, and I. Niemegeers, "Energy-efficient reliable 1702 routing considering residual energy in wireless Ad Hoc networks," IEEE 1703 Trans. Mobile Comput., vol. 13, no. 2, pp. 434-447, Feb. 2014.

[66] G. Ferrari, S. A. Malvassori, and O. K. Tonguz, "On physical layeror- 1705 iented routing with power control in Ad Hoc wireless networks," IET 1706 Commun., vol. 2, no. 2, pp. 306-319, Feb. 2008.

1707

[67] J. C. Fricke, M. M. Butt, and P. A. Hoeher, "Quality-oriented adaptive 1708 forwarding for wireless relaying," IEEE Commun. Lett., vol. 12, no. 3, 1709 pp. 200-202, Mar. 2008.

[68] M. Haenggi and D. Puccinelli, "Routing in Ad Hoc networks: A case 1711 for long hops," IEEE Commun. Mag., vol. 43, no. 10, pp. 93-101, 1712 Oct. 2005.

[69] M. Sikora, J. N. Laneman, M. Haenggi, D. J. Costello, and T. E. Fuja, 1714 "Bandwidth-and power-efficient routing in linear wireless networks," 1715 IEEE Trans. Inf. Theory, vol. 52, no. 6, pp. 2624-2633, Jun. 2006.

[70] C. Bae and W. E. Stark, "End-to-end energy and bandwidth tradeoff in 1717 multihop wireless networks," IEEE Trans. Inf. Theory, vol. 55, no. 9, 1718 pp. 4051-4066, Sep. 2009.

1719

[71] J. Niu, L. Cheng, Y. Gu, L. Shu, and S. K. Das, "R3E: Reliable reactive 1720 routing enhancement for wireless sensor networks," IEEE Trans. Ind. 1721 Informat., vol. 10, no. 1, pp. 784-794, Feb. 2014.

[72] J. Zuo, H. V. Nguyen, S. X. Ng, and L. Hanzo, "Energy-efficient relay 1723 aided Ad Hoc networks using iteratively detected irregular convolu- 1724 tional coded, unity-rate coded and space-time trellis coded transceivers," 1725 in Proc. IEEE WCNC, Quintana-Roo, Mexico, Mar. 28-31, 2011, 1726 pp. $1179-1184$.

[73] J. Zuo, C. Dong, S. X. Ng, L.-L. Yang, and L. Hanzo, "Energy-efficient 1728 routing in Ad Hoc networks relying on channel state information and 1729 limited mac retransmissions," in Proc. IEEE VTC-Fall, San Francisco, 1730 CA, USA, Sep. 5-8, 2011, pp. 1-5.

[74] J. Zuo et al., "Cross-layer aided energy-efficient opportunistic routing in 1732 Ad Hoc networks," IEEE Trans. Commun., vol. 62, no. 2, pp. 522-535, 1733 Feb. 2014

[75] D. Feng et al., "A survey of energy-efficient wireless communications," 1735 IEEE Commun. Surveys Tuts., vol. 15, no. 1, pp. 167-178, 2013.

[76] M. C. Vuran and I. F. Akyildiz, "Error control in wireless sensor net- 1737 works: A cross layer analysis," IEEE/ACM Trans. Netw., vol. 17, no. 4, 1738 pp. 1186-1199, Aug. 2009.

[77] H. V. Nguyen, S. X. Ng, and L. Hanzo, "Distributed three-stage concate- 1740 nated irregular convolutional, unity-rate and space-time trellis coding for 1741 single-antenna aided cooperative communications," in Proc. IEEE 72nd 1742 VTC-Fall, Ottawa, ON, Canada, Sep. 6-9, 2010, pp. 1-5. 1743

[78] S. T. Brink, "Convergence behavior of iteratively decoded parallel con- 1744 catenated codes," IEEE Trans. Commun., vol. 49, no. 10, pp. 1727-1737, 1745 Oct. 2001.

[79] L. Hanzo, O. Alamri, M. El-Hajjar, and N. Wu, Near-Capacity Multi- 1747 Functional MIMO Systems. New York, NY, USA: Wiley, 2009. 1748

[80] User Datagram Protocol. [Online]. Available: http://tools:ietf:org/html/ 1749 rfc768

[81] L. Hanzo, S.-X. Ng, T. Keller, and W. Webb, Quadrature Amplitude 1751 Modulation: From Basics to Adaptive Trellis-Coded, Turbo-Equalised 1752 and Space-Time Coded OFDM, CDMA, and MC-CDMA Systems, 1753 2nd ed. Hoboken, NJ, USA: Wiley-IEEE Press, 2004. 
[82] J. Zuo, S. X. Ng, and L. Hanzo, "Fuzzy logic aided dynamic source routing in cross-layer operation assisted Ad Hoc networks," in Proc IEEE 72nd VTC-Fall, Ottawa, ON, Canada, Sep. 6-9, 2010, pp. 1-5.

83] A. Ibrahim, H. Zhu, and K. J. R. Liu, "Distributed energy-efficient cooperative routing in wireless networks," IEEE Trans. Wireless Commun., vol. 7, no. 10, pp. 3930-3941, Oct. 2008.

84] E. Baccarelli, M. Biagi, C. Pelizzoni, and N. Cordeschi, "Maximumrate node selection for power-limited multiantenna relay backbones," IEEE Trans. Mobile Comput., vol. 8, no. 6, pp. 807-820, Jun. 2009.

[85] C. Bae and W. E. Stark, "End-to-end energy-bandwidth tradeoff in multihop wireless networks," IEEE Trans. Inf. Theory, vol. 55, no. 9, pp. 4051-4066, Sep. 2009

[86] S. Banerjee and A. Misra, "Minimum energy paths for reliable communication in multi-hop wireless networks," in Proc. 3rd ACM Int. Symp. MobiHoc, Lausanne, Switzerland, Jun. 9-11, 2002, pp. 146-156.

87] S. Cui, R. Madan, A. Goldsmith, and S. Lall, "Cross-layer energy and delay optimization in small-scale sensor networks," IEEE Trans. Wireless Commun., vol. 6, no. 10, pp. 3688-3699, Oct. 2007.

[88] H. Alwan and A. Agarwal, "Multi-objective QoS routing for wireless sensor networks," in Proc. ICNC, Jan. 28-31, 2013, pp. 1074-1079.

[89] X.-Y. Li et al., "Reliable and energy-efficient routing for static wireless Ad Hoc networks with unreliable links," IEEE Trans. Parallel Distrib. Syst., vol. 20, no. 10, pp. 1408-1421, Oct. 2009.

[90] M. Zorzi and R. R. Rao, "Geographic random forwarding (GeRaF) for Ad Hoc and sensor networks: Energy and latency performance," IEEE Trans. Mobile Comput., vol. 2, no. 4, pp. 349-365, Oct.-Dec. 2003.

[91] X. Mao, S. Tang, X. Xu, X.-Y. Li, and H. Ma, "Energy-efficient opportunistic routing in wireless sensor networks," IEEE Trans. Parallel Distrib. Syst., vol. 22, no. 11, pp. 1934-1942, Nov. 2011.

92] M. Dehghan, M. Ghaderi, and D. Goeckel, "Minimum-energy cooperative routing in wireless networks with channel variations," IEEE Trans. Wireless Commun., vol. 10, no. 11, pp. 3813-3823, Nov. 2011.

[93] J. Zhu and X. Wang, "Model and protocol for energy-efficient routing over mobile Ad Hoc networks," IEEE Trans. Mobile Comput., vol. 10, no. 11, pp. 1546-1557, Nov. 2011.

[94] T. Luo, M. Motani, and V. Srinivasan, "Energy-efficient strategies for cooperative multichannel MAC protocols," IEEE Trans. Mobile Comput. vol. 11, no. 4, pp. 553-566, Apr. 2012.

95] S. Kwon and N. B. Shroff, "Energy-efficient SINR-based routing for multihop wireless networks," IEEE Trans. Mobile Comput., vol. 8, no. 5 pp. 668-681, May 2009.

96] C. Wei, C. Zhi, P. Fan, and K. B. Letaief, "AsOR: An energy efficient multi-hop opportunistic routing protocol for wireless sensor networks over Rayleigh fading channels," IEEE Trans. Wireless Commun., vol. 8, no. 5, pp. 2452-2463, May 2009.

[97] M. C. Vuran and I. F. Akyildiz, "XLP: A cross-layer protocol for efficient communication in wireless sensor networks," IEEE Trans. Mobile Comput., vol. 9, no. 11, pp. 1578-1591, Nov. 2010.

[98] H. Kwon, T. H. Kim, S. Choi, and B. G. Lee, "A cross-layer strategy for energy-efficient reliable delivery in wireless sensor networks," IEEE Trans. Wireless Commun., vol. 5, no. 12, pp. 3689-3699, Dec. 2006.

[99] A. N. Pantazis, S. A. Nikolidakis, and D. D. Vergados, "Energy efficient routing protocols in wireless sensor networks: A survey," IEEE Commun. Surveys Tuts., vol. 15, no. 2, pp. 551-591, 2013.

00] M. A. Rahman, S. Anwar, M. I. Pramanik, and M. F. Rahman, "A survey on energy efficient routing techniques in wireless sensor network," in Proc. 15th ICACT, Jan. 27-30, 2013, pp. 200-205.

01] S. Biswas and R. Morris, "Opportunistic routing in multi-hop wireless networks," ACM SIGCOMM Comput. Commun. Rev., vol. 34, no. 1, pp. 69-74, Jan. 2004.

02] Q. W. Liu, S. L. Zhou, and G. B. Giannakis, "Cross-layer combining of adaptive modulation and coding with truncated ARQ over wireless links," IEEE Trans. Wireless Commun., vol. 3, no. 5, pp. 1746-1755, Sep. 2004.

03] Z. Wang, Y. Chen, and C. Li, "CORMAN: A novel cooperative opportunistic routing scheme in mobile Ad Hoc networks," IEEE J. Sel. Areas Commun., vol. 30, no. 2, pp. 289-296, Feb. 2012.

104] A. M. Akhtar, M. R. Nakhai, and A. H. Aghvami, "On the use of cooperative physical layer network coding for energy efficient routing," IEEE Trans. Commun., vol. 61, no. 4, pp. 1498-1509, Apr. 2013.

05] R. C. Shah and J. M. Rabaey, "Energy aware routing for low energy Ad Hoc sensor networks," in Proc. IEEE Wireless Commun. Netw. Conf., Mar. 2002, vol. 1, pp. 350-355.

06] Y. Xu, J. Heidemann, and D. Estrin, "Geography-informed energy conservation for Ad Hoc routing," in Proc. 7th Annu. Int. Conf. MobiCom, Rome, Italy, Jul. 2001, pp. 70-84.

Q. F. Dong and S. Banerjee, "Minimum energy reliable paths using unreliable wireless links," in Proc. 6th ACM Int. Symp. MobiHoc, UrbanaChampaign, IL, USA, May 25-28, 2005, pp. 449-459.
[108] C.-E. Perkins, E.-M. Royer, S.-R. Das, and M.-K. Marina, "Performance 1833 comparison of two on-demand routing protocols for Ad Hoc networks," 1834 IEEE Pers. Commun., vol. 8, no. 1, pp. 16-28, Feb. 2001.

[109] M. Zorzi and R. R. Rao, "Geographic random forwarding (GeRaF) 1836 for Ad Hoc and sensor networks: Multihop performance," IEEE Trans. 1837 Mobile Comput., vol. 2, no. 4, pp. 337-348, Oct.-Dec. 2003.

[110] H. Liu, B. Zhang, H. Mouftah, X. Shen, and J. Ma, "Opportunistic 1839 routing for wireless Ad Hoc and sensor networks: Present and future 1840 24 directions," IEEE Commun. Mag., vol. 47, no. 12, pp. 103-109, 1841 Dec. 2009

[111] H. Dubois-Ferrière, M. Grossglauser, and M. Vetterli, "Valuable detours: 1843 Least-cost anypath routing," IEEE/ACM Trans. Netw., vol. 19, no. 2, 1844 pp. 333-346, Apr. 2011.

[112] A. A. Bhorkar, M. Naghshvar, T. Javidi, and B. D. Rao, "Adaptive 1846 opportunistic routing for wireless Ad Hoc networks," IEEE/ACM Trans. 1847 Netw., vol. 20, no. 1, pp. 243-256, Feb. 2012.

1848

[113] K. Zeng, Z. Yang, and W. Lou, "Location-aided opportunistic forwarding 1849 in multirate and multihop wireless networks," IEEE Trans. Veh. Technol., 1850 vol. 58, no. 6, pp. 3032-3040, Jul. 2009.

[114] R. Laufer, H. Dubois-Ferrière, and L. Kleinrock, "Polynomial- 1852 time algorithms for multirate anypath routing in wireless multihop 1853 networks," IEEE/ACM Trans. Netw., vol. 20, no. 3, pp. 742-755, 1854 Jun. 2012.

[115] L. Pelusi, A. Passarella, and M. Conti, “Opportunistic networking: Data 1856 forwarding in disconnected mobile Ad Hoc networks," IEEE Commun. 1857 Mag., vol. 44, no. 11, pp. 134-141, Nov. 2006.

[116] H. Khalife, N. Malouch, and S. Fdida, "Multihop cognitive radio net- 1859 works: To route or not to route," IEEE Netw., vol. 23, no. 4, pp. 20-25, 1860 Jul. 2009.

[117] K. C. Lee, U. Lee, and M. Gerla, "Geo-opportunistic routing for vehicu- 1862 lar networks [topics in automotive networking]," IEEE Commun. Mag., 1863 vol. 48, no. 5, pp. 164-170, May 2010.

[118] D. Wu, Y. Zhang, L. Bao, and A. C. Regan, "Location-based crowd- 1865 sourcing for vehicular communication in hybrid networks," IEEE Trans. 1866 Intell. Transp. Syst., vol. 14, no. 2, pp. 837-846, Jun. 2013.

[119] V. Conan, J. Leguay, and T. Friedman, "Fixed point opportunistic routing 1868 in delay tolerant networks," IEEE J. Sel. Areas Commun., vol. 26, no. 5, 1869 pp. 773-782, Jun. 2008.

[120] T. Spyropoulos, T. Turletti, and K. Obraczka, "Routing in delaytoler- 187 ant networks comprising heterogeneous node populations," IEEE Trans. 1872 Mobile Comput., vol. 8, no. 8, pp. 1132-1147, Aug. 2009.

[121] Y. Li et al., "Energy-efficient optimal opportunistic forwarding for delay- 1874 tolerant networks," IEEE Trans. Veh. Technol., vol. 59, no. 9, pp. 4500- 1875 4512, Nov. 2010.

[122] S.-G. Yoon, S. Jang, Y.-H. Kim, and S. Bahk, "Opportunistic routing 1877 for smart grid with power line communication access networks," IEEE 1878 Trans. Smart Grid, vol. 5, no. 1, pp. 303-311, Jan. 2014.

[123] T. H. Cormen, C. E. Leiserson, R. L. Rivest, and C. Stein, Introduc- 1880 tion to Algorithms, 3rd ed. Upper Saddle River, NJ, USA: MIT Press, 1881 2009.

[124] E. W. Dijkstra, "A note on two problems in connexion with graphs," 1883 Numerische Mathematik, vol. 1, no. 1, pp. 269-271, 1959.

[125] M. L. Fredman and R. E. Tarjan, "Fibonacci heaps and their uses in 1885 improved network optimization algorithms," J. Assoc. Comput. Mach., 1886 vol. 34, no. 3, pp. 596-615, Jul. 1987.

[126] C. Dong, L.-L. Yang, and L. Hanzo, "Multi-hop diversity aided multi- 1888 hop communications: A cumulative distribution function aware ap- 1889 proach," IEEE Trans. Commun., vol. 61, no. 11, pp. 4486-4499, 1890 Nov. 2013.

[127] C. Dong, L.-L. Yang, and L. Hanzo, "Performance analysis of multihop- 1892 diversity-aided multihop links," IEEE Trans. Veh. Technol., vol. 61, 1893 no. 6, pp. 2504-2516, Jul. 2012.

[128] W. C. Tan, S. K. Bose, and T.-H. Cheng, "Power and mobility aware 1895 routing in wireless Ad Hoc networks," IET Commun., vol. 6, no. 11, 1896 pp. 1425-1437, Jul. 2012.

1897

[129] F. D. Rango, F. Guerriero, and P. Fazio, "Link-stability and energy aware 1898 routing protocol in distributed wireless networks," IEEE Trans. Parallel 1899 Distrib. Syst., vol. 23, no. 4, pp. 713-726, Apr. 2012.

[130] A. A. Jeng and R.-H. Jan, "Adaptive topology control for mobile Ad Hoc 190 networks," IEEE Trans. Parallel Distrib. Syst., vol. 22, no. 12, pp. 1953- 1902 1960, Dec. 2011.

[131] G. Ferrari and O. K. Tonguz, "Impact of mobility on the BER perfor- 1904 mance of Ad Hoc wireless networks," IEEE Trans. Veh. Technol., vol. 56, 1905 no. 1, pp. 271-286, Jan. 2007.

1906

[132] S. L. Correia, J. Celestino, and O. Cherkaoui, "Mobility-aware 1907 ant colony optimization routing for vehicular Ad Hoc networks," in 1908 Proc. IEEE WCNC, Quintana-Roo, Mexico, Mar. 28-31, 2011, 1909 pp. 1125-1130. 


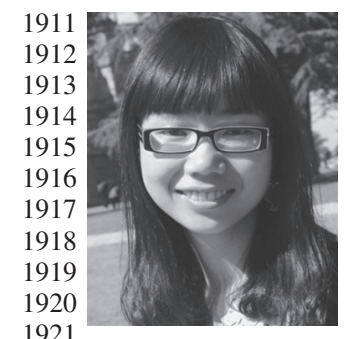

Jing Zuo received the B.Eng. degree in communications engineering and the M.Sc. degree in communications and information system from Jilin University, Changchun, China, in 2006 and 2008, respectively, and the Ph.D. degree in wireless communications from University of Southampton, U.K., in 2013. She is the recipient of scholarship under the UK-China Scholarships for Excellence programme from 2008 to 2011. From 2009 to 2013, she was involved in the OPTIMIX and CONCERTO European projects. She is currently with Huawei, Shenzhen, China and her 1922 current research interests include protocols and algorithms design, cross-layer 1923 optimization and opportunistic communications.

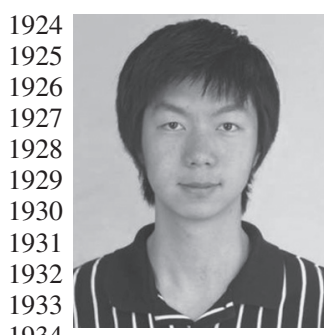

Chen Dong received the B.S. degree in electronic information sciences and technology from University of Science and Technology of China (USTC), Hefei, China, in 2004, and the M.Eng. degree in pattern recognition and automatic equipment from the University of Chinese Academy of Sciences, Beijing, China in 2007. He received the Ph.D. degree from the University of Southampton, UK, In 2014. Now he is a post-doc in the same University. He was the recipient of scholarship under the UK-China Scholarships for Excellence programme and he has been awarded 1935 Best Paper Award at IEEE VTC 2014-Fall. His research interests include 1936 applied math, relay system, channel modelling and cross-layer optimization.

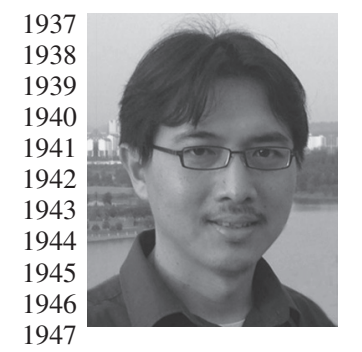

Soon Xin Ng (S'99-M'03-SM'08) received the B.Eng. degree (first class) in electronic engineering and the Ph.D. degree in telecommunications from the University of Southampton, Southampton, U.K., in 1999 and 2002, respectively. From 2003 to 2006, he was a postdoctoral research fellow working on collaborative European research projects known as SCOUT, NEWCOM and PHOENIX. Since August 2006, he has been a member of academic staff in the School of Electronics and Computer Science, University of Southampton. He is involved in the 1948 OPTIMIX and CONCERTO European projects as well as the IU-ATC and 1949 UC4G projects. He is currently an associate professor in telecommunications at 1950 the University of Southampton.

1951 His research interests include adaptive coded modulation, coded modula1952 tion, channel coding, space-time coding, joint source and channel coding, 1953 iterative detection, OFDM, MIMO, cooperative communications, distributed 1954 coding, quantum error correction codes and joint wireless-and-optical-fiber 1955 communications. He has published over 180 papers and co-authored two John 1956 Wiley/IEEE Press books in this field. He is a Chartered Engineer and a Fellow 1957 of the Higher Education Academy in the U.K.

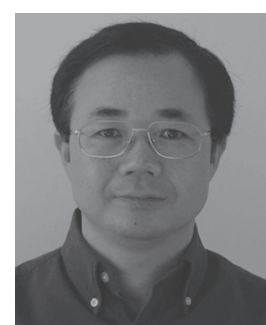

Lie-Liang Yang (M'98-SM'02) received the 1958 B.Eng. degree in communications engineering from 1959 Shanghai TieDao University, Shanghai, China, 1960 in 1988, and the M.Eng. and Ph.D. degrees in 1961 communications and electronics from Northern 1962 (Beijing) Jiaotong University, Beijing, China in 1963 1991 and 1997, respectively. From June 1997 to 1964 December 1997 he was a visiting scientist of the 1965 Institute of Radio Engineering and Electronics, 1966 Academy of Sciences of the Czech Republic. Since 1967 December 1997, he has been with the University 1968 of Southampton, United Kingdom, where he is the professor of wireless 1969 communications in the School of Electronics and Computer Science. His 1970 research has covered a wide range of topics in wireless communications, 1971 networking and signal processing. He has published over 300 research papers 1972 in journals and conference proceedings, authored/co-authored three books 1973 and also published several book chapters. The details about his publications 1974 can be found at http://www-mobile.ecs.soton.ac.uk/lly/. He is a Fellow of 1975 the IET, served as an associate editor to the IEEE TRANS. ON VEHICULAR 1976 TECHNOLOGY and Journal OF COMmuniCATIONS AND NeTwORKS 1977 (JCN), and is currently an associate editor to the IEEE Access and the Security 1978 and Communication Networks (SCN) Journal.

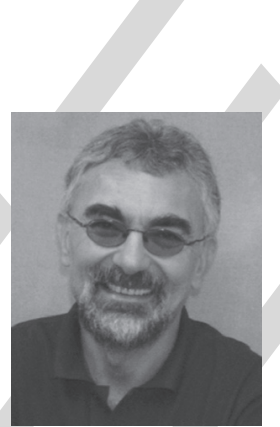

Lajos Hanzo received the degree in electronics in 1980 1976 and the doctorate degree in 1983. In 2009 he 1981 was awarded the honorary doctorate "Doctor Hon- 1982 oris Causa" by the Technical University of Budapest. 1983 During his 37-year career in telecommunications he 1984 has held various research and academic posts in 1985 Hungary, Germany and the UK. Since 1986 he has 1986 been with the School of Electronics and Computer 1987 Science, University of Southampton, UK, where he 1988 holds the chair in telecommunications. He has suc- 1989 cessfully supervised more than 80 Ph.D. students, 1990 co-authored 20 John Wiley/IEEE Press books on mobile radio communications 1991 totalling in excess of 10000 pages, published 1460 research entries at IEEE 1992 Xplore, acted both as TPC and General Chair of IEEE conferences, presented 1993 keynote lectures and has been awarded a number of distinctions. Currently 1994 he is directing a 60-strong academic research team, working on a range of 1995 research projects in the field of wireless multimedia communications sponsored 1996 by industry, the Engineering and Physical Sciences Research Council (EPSRC) 1997 UK, the European Research Council's Advanced Fellow Grant and the Royal 1998 Society's Wolfson Research Merit Award. He is an enthusiastic supporter of 1999 industrial and academic liaison and he offers a range of industrial courses. He 2000 is also a Governor of the IEEE VTS. During 2008-2012 he was the Editor-in- 2001 Chief of the IEEE Press and a Chaired Professor also at Tsinghua University, 2002 Beijing. His research is funded by the European Research Council's Senior 2003 Research Fellow Grant. For further information on research in progress and 2004 associated publications please refer to http://www-mobile.ecs.soton.ac.uk. 2005 


\section{AUTHOR QUERIES}

\section{AUTHOR PLEASE ANSWER ALL QUERIES}

AQ1 = Note that reference [26] and [105] are the same. Therefore, reference [105] was deleted from the list. Citations were renumbered accordingly. Please check.

AQ2 = Note that reference [57] and [109] are the same. Therefore, reference [109] was deleted from the list. Citations were renumbered accordingly. Please check.

\section{END OF ALL QUERIES}




\title{
Cross-Layer Aided Energy-Efficient Routing Design for Ad Hoc Networks
}

\author{
Jing Zuo, Chen Dong, Soon Xin Ng, Lie-Liang Yang, and Lajos Hanzo
}

\begin{abstract}
4 Abstract-In this treatise, we first review some basic routing 5 protocols conceived for ad hoc networks, followed by some design 6 examples of cross-layer operation aided routing protocols. Specif7 ically, cross-layer operation across the PHYsical layer (PHY), 8 the Data Link layer (DL) and even the NETwork layer (NET)

9 is exemplified for improving the energy efficiency of the entire 10 system. Moreover, the philosophy of Opportunistic Routing (OR) 11 is reviewed for the sake of further reducing the system's energy 12 dissipation with the aid of optimized Power Allocation (PA). The 13 system's end-to-end throughput is also considered in the context 14 of a design example.
\end{abstract}

15 Index Terms-Opportunistic routing, cross-layer, objective func16 tion, near-capacity coding, energy consumption, power allocation.

\section{INTRODUCTION}

18 INCE the commencement of the Defense Advanced Re19 search Projects Agency (DARPA) project [1] developed 20 by the American Defense Department in the 1970s, ad hoc 21 networks have been widely applied in scenarios, including 22 military applications, crisis response, medical care, conference 23 meetings and space exploration. During the past few decades, 24 ad hoc networks attracted substantial research attention as a 25 benefit of their prompt set-up and their ability to self-organize 26 their noncentrally-controlled dynamic topology. Each node of 27 an ad hoc network plays the dual role of being both a terminal 28 and a router under the assumption that not all nodes can directly 29 communicate with each other [2]. Fig. 1(a) and (b) show the 30 difference between the classic infrastructure based network and 31 ad hoc network. Fig. 1(a) shows that the nodes $A, B$ and $C$ 32 communicate with each other under the control of Base Station 33 (BS) 1 and that $A$ communicates with $E$ via BS 1 and BS 2. 34 However, Fig. 1(b) shows that $A$ can only communicate with $E$ 35 by relying on $B, C$ and $D$ as its Relay Nodes (RNs). Each node 36 has to discover its own neighbor list.

37 The characteristics of ad hoc networks impose a number of 38 open problems, which constitute challenges for the protocol 39 design. For example, the scalability, the energy-efficiency, the

Manuscript received January 9, 2014; revised July 6, 2014 and October 31, 2014; accepted January 17, 2015. The research leading to these results has received funding from the European Union's Seventh Framework Programme (FP7/2012-2014) under grant agreement no 288502. The financial support of the China-UK Scholarship Council, and of the RC-UK under the auspices of the IU-ATC initiative is also gratefully acknowledged.

The authors are with the School of Electronics and Computer Science (ECS), University of Southampton, Southampton SO17 1BJ, U.K. (e-mail: jz08r@ecs. soton.ac.uk; cd2g09@ecs.soton.ac.uk; sxn@ecs.soton.ac.uk; 1ly@ecs.soton. ac.uk; 1h@ecs.soton.ac.uk; http://www-mobile.ecs.soton.ac.uk).

Color versions of one or more of the figures in this paper are available online at http://ieeexplore.ieee.org.

Digital Object Identifier 10.1109/COMST.2015.2395378

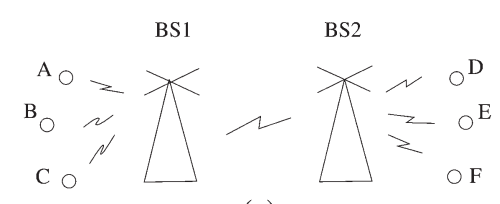

(a)

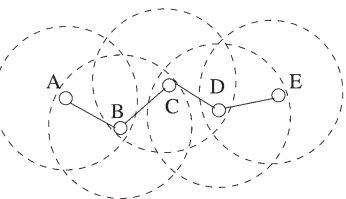

(b)

Fig. 1. Categories of wireless networks. (a) The infrastructure network; (b) Ad hoc network.

Quality of Service (QoS) and the security are challenging 40 problems to be solved and to be further improved. Hence the 41 emphasis of this treatise is on the design of routing protocols 42 relying on cross-layer interaction for improving the attainable 43 system performance, such as the Normalized Energy Consump- 44 tion (NEC) and the end-to-end throughput.

A. Cross-Layer Design

The International Standards Organization (ISO) created the 47 SubCommittee 16 (SC16) in 1977 for developing an architec- 48 ture, which could serve as a framework for the definition of 49 standard protocols. At the end of 1979, the Reference Model 50 of Open System Interconnection (OSI) was adopted by the 51 parent of SC16, namely, Technical Committee (TC97). The 52 OSI Reference Model was also recognized by the International 53 Telegraph and Telephone Consultative Committee (CCITT) 54 Rappporteur's Group on Public Data Network Services. The 55 OSI Reference Model consists of seven layers, which are the 56 PHYsical layer (PHY), the Data Link layer (DL), the NET- 57 work layer (NET), the transport layer, the session layer, the 58 presentation layer and the application layer. The benefit of 59 this layering technique is to group the similar communication 60 functions into these logical layers. A layer has to cooperate with 61 the layer above it and the layer below it. However, when the 62 Transmission Control Protocol (TCP) of the transport layer and 63 the Internet Protocol (IP) of the network layer were defined, 64 the five-layer model (TCP/IP model) became the dominant one. 65 More explicitly, the TCP/IP model consists of the application 66 layer, the transport layer, the NETwork (NET) layer, the Data 67 Link (DL) layer and the PHYsical (PHY) layer [3]-[6]. Fig. 268 illustrates the structure of the TCP/IP model and the main 69 functions of each layer.

The functions of these layers are briefly highlighted below: 71

- The physical layer: The PHY layer concentrates on both 72 the physical devices and on the transmission media. Pro- 73 viding a diversity and/or multiplexing gain with the aid 74 of multiple antennas is capable of improving the integrity 75 and/or throughput of data transmission. Additionally, the 76 


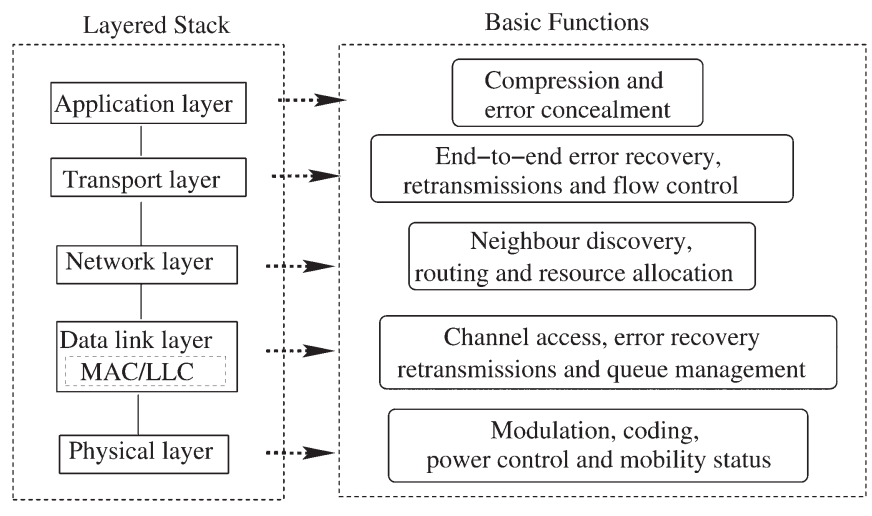

Fig. 2. Layered stack and the main functions in each layer. 111 vantages and performs well in wired networks in terms of 112 portability, flexibility and low design complexity, it is not 113 suitable for wireless networks, especially in wireless ad hoc 114 networks. The reason for its inadequacy in wireless scenarios 115 is that the services offered by the layers to those above them 116 in Fig. 2 are realized by specifically tailored protocols for the 117 different layers and that the architecture forbids direct com118 munication between non-adjacent layers. The communication 119 between adjacent layers is limited to procedure calls and to their

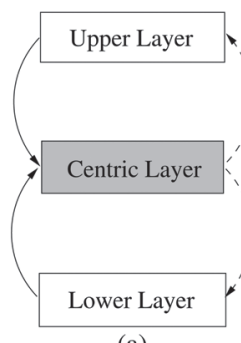

(a)

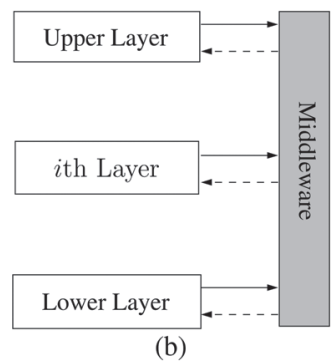

Fig. 3. Conceptual illustration of cross-layer design methods. (a) Layercentric solution; (b) centralized solution.

responses. Moreover, the hostile wireless links impose several 120 new problems on the associated protocol design that cannot 121 be readily handled by the layered architecture [13]. More 122 explicitly, having a strict layered design is not flexible enough to 123 cope with the dynamics of Mobile Ad hoc NETwork (MANET) 124 environments and will thus result in a low performance [14]. 125 Thus, the mutual impact of the layers on each other cannot 126 be ignored [15]. Hence the concept of cross-layer design has 127 been proposed in an attempt to achieve a performance gain by 128 exploiting the close interaction amongst the different layers. 129 Srivastava and Motani [13] defined "Cross-Layer operation" 130 as: "Protocol design by the violation of a reference layered 131 communication architecture is cross-layer design with respect 132 to the particular layered architecture", while Jurdak [15] define 133 it as: "Cross-layer design with respect to a reference layered 134 architecture is the design of algorithms, protocols, or architec- 135 tures that exploit or provide a set of inter-layer interactions that 136 is a superset of the standard interfaces provided by the reference 137 layered architecture". Therefore, cross-layer operation may be 138 interpreted as the 'violation' of the layered architecture seen 139 in Fig. 2, which requires more interaction amongst the layers, 140 beyond the interaction between the adjacent layers. Cross-layer 141 design clearly requires information exchange between layers, as 142 well as adaptivity to this information at each layer and a certain 143 grade of diversity built into each layer for the sake of improving 144 the achievable robustness [5].

145

There are two basic methods of information sharing in cross- 146 layer design [16]. One of them makes the variables of a specific 147 layer visible to the other layers, which is referred to as a layer- 148 centric solution. The other relies on a shared middleware [15], 149 [16], which provides the service of storage/retrieval of infor- 150 mation to all layers, which is termed as a centralized solution. 151 Fig. 3 illustrates how these two cross-layer solutions operate. 152

The basic principles of the above-mentioned pair of cross- 153 layer solutions are:

- The layer-centric solution: A certain layer is allowed to 155 be the central layer, which controls the cross-layer adap- 156 tation by accessing the internal protocol parameters and 157 algorithms of the other layers, as shown in Fig. 3(a). Al- 158 though this approach significantly improves the attainable 159 system performance, it violates the layered architecture, 160 since it requires access to the internal variables of other 161 layers.

- The centralized solution: A middleware or a system-level 163 monitor (centralized optimizer) is employed for estimating 164 both the availability of resources and the environmental 165 
TABLE I

Major Contributions of Cross-Layer Design in Ad Hoc Networks

\begin{tabular}{|l|l|l|}
\hline Year & Authors & Contribution \\
\hline \hline 2005 & Setton et al. [18] & $\begin{array}{l}\text { Explored the potential synergies of exchanging information between different layers to support real-time } \\
\text { video streaming. }\end{array}$ \\
\hline 2006 & Liu et al. [19] & $\begin{array}{l}\text { Proposed a scheduling algorithm at the MAC layer for multiple connections under diverse QoS } \\
\text { requirements, where each connection employs both adaptive modulation and coding at the PHY layer } \\
\text { for transmission over wireless channels. }\end{array}$ \\
\hline 2007 & $\begin{array}{l}\text { Huang } \\
\text { and Letaief [20] }\end{array}$ & $\begin{array}{l}\text { Proposed a cross-layer optimization framework to jointly design the scheduling, power control and } \\
\text { adaptive modulation. }\end{array}$ \\
\hline 2008 & $\begin{array}{l}\text { Zhang } \\
\text { and Zhang [21] }\end{array}$ & $\begin{array}{l}\text { Reviewed the state-of-the-art on the cross-layer paradigm and discussed the open issues related to cross- } \\
\text { layer design for QoS support. }\end{array}$ \\
\hline 2009 & $\begin{array}{l}\text { Oh } \\
\text { and Chen [22] }\end{array}$ & $\begin{array}{l}\text { Presented a cross-layer design for reliable video transmission based on a multichannel MAC protocol in } \\
\text { the context of time division multiple access. }\end{array}$ \\
\hline 2010 & $\begin{array}{l}\text { Chu } \\
\text { and Wang [23] }\end{array}$ & $\begin{array}{l}\text { Presented cross-layer centralized and distributed scheduling algorithms, which exploited the PHY layer } \\
\text { channel information to opportunistically schedule cooperative spatial multiplexed transmissions between } \\
\text { MIMO-based nodes. }\end{array}$ \\
\hline 2011 & $\begin{array}{l}\text { Ghosh } \\
\text { and Hamouda [24] }\end{array}$ & $\begin{array}{l}\text { Proposed a cross-layer antenna selection algorithm for improving the transmission efficiency in cognitive } \\
\text { MIMO-aided ad hoc networks. }\end{array}$ \\
\hline 2012 & Mardani et al. [25] & $\begin{array}{l}\text { Jointly considered flow control, multipath routing and random access control based on network utility } \\
\text { maximization. }\end{array}$ \\
\hline 2013 & Uddin et al. [26] & $\begin{array}{l}\text { Studied cross-layer design in random-access-based fixed wireless multihop networks under a physical } \\
\text { interference model. }\end{array}$ \\
\hline 2014 & $\begin{array}{l}\text { Tang } \text { et al. [27] } \\
\text { Proposed a cross-layer distributed approach for maximizing the network throughput by jointly selecting } \\
\text { stable routes and assigning channels based on mobility prediction. }\end{array}$ \\
\hline \hline
\end{tabular}

178 The cross-layer operation aided design of wireless ad hoc 179 networks poses challenges mainly due to the time-variant char180 acteristics of wireless channels. The signal is substantially more 181 vulnerable to the effects of noise, fading and interference than 182 in benign fixed networks, leading to potential performance 183 degradations within the higher layers. For example, a packet has 184 to be retransmitted in the DL layer or the transmit power has to 185 be adjusted to guarantee its high-integrity transmission, which 186 may impose interference on other nodes or promote aggressive 187 contention for channel access. In the NET layer, the current 188 route may become invalid and hence route maintenance/repair 189 has to be activated or even a new route discovery process has to 190 be initiated. As a result, potentially more energy is consumed 191 and the end-to-end delay is increased, while the end-to-end 192 throughput is reduced. Therefore, careful adaptation of the 193 protocol stack should be used at each layer to compensate for 194 the variations at that layer, depending on the specific time scale 195 of these variations [5]. Both the local adaptation of parameters 196 within each layer and the adaptation based on the other layers 197 have to be considered. For example, the transmit power, the 198 signal processing hardware's power dissipation, the information 199 transmit rate, the coding and modulation schemes, the Frame 200 Error Ratio (FER) and the mobility in the PHY layer consti- tute important parameters, which may be beneficially shared 201 with other layers. The protocol design of the upper layers has 202 to consider the information gleaned from the PHY layer for 203 minimizing the energy consumption, the resource allocation, 204 scheduling and the queueing management, while maintaining 205 a certain QoS guarantee. Meanwhile, the number of retransmis- 206 sions, as well as both the routing and network topology related 207 information received from the upper layers may be beneficially 208 shared. Additionally, node cooperation also calls for cross-layer 209 design [13]. Table I is presented for discussing the previous 210 work on cross-layer design in a compact manner.

These cross-layer aided designs may be classified into several 212 categories according to their different application requirements. 213 They might be designed for reducing the energy consumption, 214 the end-to-end delay [21], for improving the network's through- 215 put [18], [22], [24], [26], [27], for striking a flexible tradeoff 216 between any two of them [20], [23], and even for multiple- 217 constraint optimization [19], [21].

As detailed above, cross-layer design has substantial benefits, 219 but it has its own disadvantages as well. For example, the cross- 220 layer interactions create dependencies amongst the layers, 221 which will affect not only the layer concerned, but also the other 222 layers. Hence, a complete redesign of the operational networks 223 and protocols will lead to a high implementational cost [16]. 224 Therefore, cross-layer design should be carefully crafted, be- 225 cause once the seven-layer OSI structure is violated, the benefits 226 of independent, layer-specific protocol design will disappear 227 [13], [28]. The effects of any protocol chosen in every single 228 layer on the overall system has to be carefully considered.

\section{B. Categories of Ad Hoc Routing Protocols}

The NET layer of Fig. 2 plays a key role in ad hoc networks, 231 which substantially influences the performance of the overall 232 system. The NET layer is responsible both for allocating IP 233 


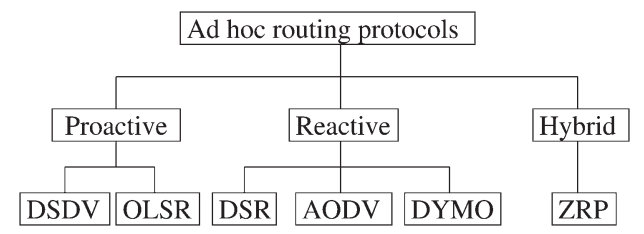

Fig. 4. Categorization of ad hoc routing protocols (DSDV: DestinationSequenced Distance Vector routing; OLSR: Optimized Link State Routing; DSR: Dynamic Source Routing; AODV: Ad-hoc On-demand Distance Vector routing; DYMO: DYnamic Manet On-demand routing; ZRP: Zone Routing Protocol).

234 addresses and for choosing the right route for communication 235 between the source and destination. The routing protocols of $\mathrm{ad}$ 236 hoc networks may be classified as proactive routing, reactive 237 routing and hybrid routing [29], as shown in Fig. 4.

238 The proactive routing periodically transmits "hello" packets 239 for the sake of identifying all possible routes in the network. 240 Hence every node has to maintain a routing table, which stores 241 the route spanning from itself to all other available nodes. The 242 advantage of this kind of routing protocol is that the route 243 discovery time is low. By contrast, its disadvantage is that each 244 node has to maintain a routing table. If the number of nodes in 245 the network becomes high, then the routing table becomes large 246 and hence requires a large memory. On the other hand, periodi247 cally sending "hello" packets increases the network control load 248 imposed. The so-called Destination-Sequenced Distance Vector 249 (DSDV) [30] protocol and the Optimized Link State Routing 250 (OLSR) [31] protocol are typical proactive routing protocols, 251 as seen in Fig. 4.

252 The reactive routing protocols are source-driven, implying 253 that they transmit route discovery packets to find a route to 254 the destination, when there is sufficient data scheduled for 255 transmission in the buffer, instead of periodically broadcasting 256 the "hello" packets. As a benefit, not all nodes have to maintain 257 a route table for storing the routes leading to all other nodes. In258 stead, they only store routes that were found during the process 259 of route discovery. This technique reduces the network control 260 load compared to the proactive routing protocols of Fig. 4. The 261 disadvantage of this routing protocol family is however that 262 their delay is increased, because a route has to be found to the 263 destination, when no routes leading to the destination exist in 264 the route table. Additionally, the nodes' movement changes the 265 network's topology, which hence requires the transmission of 266 more control packets for the sake of maintaining the current 267 communication session. As seen in Fig. 4, the Dynamic Source 268 Routing (DSR) [32], Ad-hoc On-demand Distance Vector 269 (AODV) [33] and DYnamic Manet On-demand (DYMO) [34] 270 routing protocols constitute typical reactive routing protocols.

271 Based on beneficially combining the advantages, whilst 272 avoiding the disadvantages of the above-mentioned protocol 273 families, hybrid routing protocols may also be conceived. We 274 may divide the entire ad hoc network into several small areas 275 and in each area proactive routing may be employed for estab276 lishing a link for all nodes. By contrast, between the areas, reac277 tive routing protocols may be adopted for reducing the number 278 of control packets required. Hybrid protocols are widely applied 279 in large ad hoc networks. The so-called Zone Routing Protocol 280 (ZRP) [35] is a typical hybrid routing protocol.

\section{Review of Cross-Layer Aided Routing Protocols}

This treatise is mainly dedicated to cross-layer operation 282 aided routing design in ad hoc networks, hence we list the 283 major contributions to the literature of cross-layer aided routing 284 protocols conceived for ad hoc networks in Table II.

285

Similarly, these cross-layer aided routing protocols may be 286 classified into several categories according to their different 287 application requirements. They might be designed for reducing 288 the energy consumption [38], [48], [52], the end-to-end delay 289 [47], for improving the network's throughput [39], [49], [54], 290 for striking a flexible tradeoff between any two of them [26], 291 [42], [45], [46], [50], [51], and even for multiple-constraint 292 optimization [36], [41], [43], [44].

\section{Review of Energy-Efficient Routing Protocols}

As mentioned in Section I-C, cross-layer design may be 295 studied based on diverse application requirements. This paper 296 focuses on cross-layer design techniques conceived for reduc- 297 ing the energy consumption. since energy saving in wireless 298 ad hoc networks is of salient importance in the interest of 299 mitigating the problem of limited battery supply at each 300 node. In ad hoc networks the nodes actively and voluntarily 301 participate in constructing a network and act as relays for 302 other nodes. As a result of node-mobility, the Channel State 303 Information (CSI) varies and hence a substantial amount 304 of control messages have to be exchanged across the net- 305 work to maintain reliable communications between certain 306 pairs of nodes, which potentially imposes a high energy- 307 consumption. Therefore, minimizing the energy consump- 308 tion becomes extremely important. Numerous power-aware 309 routing protocols were proposed in [55] for improving the 310 energy efficiency from a multiuser networking perspective. 311 Firstly, a compact-form review of energy-efficient single-layer 312 routing design is provided in Table III.

Moreover, cross-layer optimized power control has been 314 widely exploited [66]-[71] for maintaining the required target- 315 integrity at a low power in realistic propagation environments. 316 A physical-layer-oriented routing protocol supported by sophis- 317 ticated power control was proposed in [66] for a Line-Of-Sight 318 (LOS) and shadow faded scenario, where the estimated end-to- 319 end BER of a multi-hop path was used as the route selection 320 metric. Furthermore, an adaptive relaying strategy switching 321 between the Amplify-and-Forward (AF) and the Decode-and- 322 Forward (DF) schemes was proposed in [67] for reducing both 323 the energy consumption as well as the delay of the system. As 324 a further design dilemma, the influence of the 'small number 325 of long hops' versus the 'many short hops' philosophy on the 326 energy consumption was studied in [68]-[70]. It was indicated 327 in [68] that the 'small number of long hops' routing scheme 328 was better than the 'many short hops' routing scheme provided 329 that near-capacity coding strategies combined with a relatively 330 short block length were employed, because a substantial SNR 331 loss was exhibited by the 'many short hops' based routing 332 scheme. Moreover, it was demonstrated in [69] that 'many 333 short hops' perform well in energy-limited scenarios relying on 334 spatial reuse, even in the absence of interference cancellation, 335 while using a 'small number of long hops' is more suitable for 336 
TABLE II

Major Contributions of Cross-Layer Aided Routing Protocols in Ad Hoc Networks

\begin{tabular}{|c|c|c|}
\hline Year & Authors & Contribution \\
\hline 2002 & $\begin{array}{l}\text { Goldsmith } \\
\text { and Wicker [36] }\end{array}$ & $\begin{array}{l}\text { Reviewed each layer's protocol and emphasized the necessity of cross-layer design, particularly in energy- } \\
\text { limited scenarios. }\end{array}$ \\
\hline \multirow[t]{2}{*}{2005} & Souryal et al. [37] & Proposed efficient channel-quality-aware adaptive routing relying on adaptive modulation. \\
\hline & Lee et al. [38] & Combined power-aware routing with a MAC layer algorithm for minimizing the total consumed power. \\
\hline \multirow[t]{4}{*}{2006} & $\begin{array}{l}\text { Johansson } \\
\text { and Xiao [39] }\end{array}$ & $\begin{array}{l}\text { Jointly optimized the end-to-end communication rates, routing, power allocation and transmission } \\
\text { scheduling of a network. }\end{array}$ \\
\hline & Mao et al. [40] & $\begin{array}{l}\text { proposed a Genetic Algorithm (GA)-based application-centric cross-layer approach for minimizing video } \\
\text { distortion. }\end{array}$ \\
\hline & $\begin{array}{l}\text { Abdrabou } \\
\text { and Zhuang [41] }\end{array}$ & Presented a position-aware QoS routing scheme by considering its interactions with the MAC. \\
\hline & Zhang et al. [42] & $\begin{array}{l}\text { Addressed the topic of energy-efficient routing subject to both packet delay and multi-access interference } \\
\text { constraints. }\end{array}$ \\
\hline \multirow[t]{2}{*}{2007} & $\begin{array}{l}\text { Kompella } \\
\text { et al. }[43]\end{array}$ & $\begin{array}{l}\text { Optimized the performance of Multiple Description (MD) video subject to certain routing and link layer } \\
\text { constraints. }\end{array}$ \\
\hline & $\begin{array}{l}\text { Chiang } \\
\text { et al. }[44]\end{array}$ & $\begin{array}{l}\text { Surveyed the functional modules, such as congestion control, routing, scheduling, random access, power } \\
\text { control and channel coding. }\end{array}$ \\
\hline \multirow[t]{2}{*}{2008} & Phan et al. [45] & $\begin{array}{l}\text { Presented a cross-layer optimization approach jointly considering the design of the MAC, routing and } \\
\text { energy distribution. }\end{array}$ \\
\hline & Liu et al. [46] & $\begin{array}{l}\text { Jointly optimized the power and bandwidth allocation at each node and designed multihop/multipath } \\
\text { routing for a MIMO-based wireless } a d \text { hoc network. }\end{array}$ \\
\hline \multirow[t]{2}{*}{2009} & $\begin{array}{l}\text { Abdrabou } \\
\text { and Zhuang [47] }\end{array}$ & $\begin{array}{l}\text { Proposed a routing scheme based on a geographical on-demand routing protocol, which is capable of } \\
\text { guaranteeing a certain maximum end-to-end delay. }\end{array}$ \\
\hline & Li et al. $[48]$ & $\begin{array}{l}\text { Proposed a combined multi-rate power controlled MAC protocol and routing protocol relying on the } \\
\text { effective transport capacity as the routing metric. }\end{array}$ \\
\hline \multirow[t]{2}{*}{2010} & Ding et al. [49] & $\begin{array}{l}\text { Proposed a ROuting and dynamic Spectrum-Allocation (ROSA) algorithm aiming for maximizing the } \\
\text { network's throughput by performing joint routing, dynamic spectrum allocation, scheduling and } \\
\text { transmit power control. }\end{array}$ \\
\hline & Lu et al. [50] & $\begin{array}{l}\text { Presented Joint Channel Assignment and Cross-layer Routing (JCACR) by employing two metrics, namely } \\
\text { the Channel Utilization Percentage (CUP) and the Channel Selection Metric (CSM). }\end{array}$ \\
\hline \multirow[t]{2}{*}{2011} & $\begin{array}{l}\text { Ding } \\
\text { and Leung [51] }\end{array}$ & $\begin{array}{l}\text { Proposed cross-layer routing applying both cooperative transmission and path selection for striking a } \\
\text { tradeoff between the transmit power consumption and the end-to-end reliability. }\end{array}$ \\
\hline & $\begin{array}{l}\text { Tavli and } \\
\text { Heinzelman [52] }\end{array}$ & Presented real-time multicasting based routing. \\
\hline 2012 & Syue et al. [53] & Proposed a relay-aware cooperative routing protocol relying on cross-layer design. \\
\hline 2013 & Pan et al. [54] & $\begin{array}{l}\text { Investigated the path selection problem based on the cross-layer optimization in on flow routing, multihop } \\
\text { Cognitive Radio Networks(CRNs) under constraints link scheduling and CR source's budget. }\end{array}$ \\
\hline 2014 & Uddin et al. [26] & $\begin{array}{l}\text { Studied cross-layer design in random-access-based fixed wireless multihop networks under a physical } \\
\text { interference model. }\end{array}$ \\
\hline
\end{tabular}

TABLE III

Major Contributions of Single Layer Energy-Efficient Routing Protocols in Ad Hoc Networks

\begin{tabular}{|l|l|l|}
\hline Year & Authors & Contribution \\
\hline \hline 2005 & Muruganathan et al. [56] & $\begin{array}{l}\text { Proposed a centralized routing protocol referred to as a base-station controlled dynamic clustering } \\
\text { protocol, which distributes the energy dissipation evenly among all sensor nodes for improving the } \\
\text { network lifetime and for achieving average energy savings. }\end{array}$ \\
\hline 2006 & Zhu et al. [57] & $\begin{array}{l}\text { Proposed a minimum energy dissipation routing protocol based on an accurate model, which took } \\
\text { into account the energy consumption of both the data packets, as well as of the control packets and } \\
\text { retransmissions. }\end{array}$ \\
\hline 2007 & $\begin{array}{l}\text { Baek } \\
\text { and Veciana [58] }\end{array}$ & $\begin{array}{l}\text { Investigated the employment of proactive multipath routing to achieve a tradeoff between the energy } \\
\text { cost of spreading traffic and the improved spatial balance of energy. }\end{array}$ \\
\hline 2008 & Eidenbenz et al. [59] & $\begin{array}{l}\text { Designed an energy-efficient distributed algorithm based on the so-called side payment scheme in } \\
\text { conjunction with a game-theoretic technique to achieve truthfulness for the rational selfish nodes. }\end{array}$ \\
\hline 2009 & Liang et al. [60] & $\begin{array}{l}\text { Designed energy-efficient routing algorithms, which constructed a shared multicast tree spanning all } \\
\text { terminal nodes, while ensuring that the total energy consumption of realizing all-to-all multicasting } \\
\text { was minimized. }\end{array}$ \\
\hline 2011 & $\begin{array}{l}\text { Ma } \text { et al. } \text { [61] } \\
\text { and Yang [62] }\end{array}$ & $\begin{array}{l}\text { Proposed a virtual-link-reduction-based broadcasting protocol using directional antennas. } \\
\text { Proposed an online computable discrete-time mathematical energy model for characterising the } \\
\text { battery discharging behavior and proposed a battery-aware routing schemethat incorporates battery } \\
\text { awareness into routing protocols. }\end{array}$ \\
\hline 2012 & $\begin{array}{l}\text { Akhtar } \text { et al. } \text { [63] } \\
\text { Designed a cooperative routing algorithm, which took the electronic power consumption into } \\
\text { consideration, when constructing the minimum-power route leading from source to destination. }\end{array}$ \\
\hline 2013 & $\begin{array}{l}\text { Lu } \\
\text { and Zhu [64] }\end{array}$ & $\begin{array}{l}\text { Proposed an energy-efficient genetic algorithm aided mechanism, which depended on bounded end- } \\
\text { to-end delay and minimum energy cost of the multicast tree, to solve QoS based multicast routing } \\
\text { problems. }\end{array}$ \\
\hline \hline
\end{tabular}




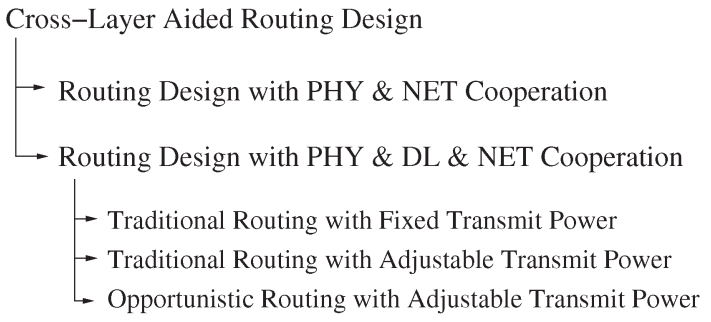

Fig. 5. Structure of this treatise.

337 bandwidth-limited scenarios. Therefore, the routing algorithms 338 should be carefully designed, when jointly considering both 339 the achievable energy-efficiency and the attainable bandwidth340 efficiency. The tradeoffs between energy- and bandwidth341 efficiency were studied in [70], where it was found that at high 342 end-to-end data rates the routes associated with fewer hops 343 minimize the energy consumption, while at lower end-to-end 344 data rates the routes having more hops mitigate it.

\section{E. Outline}

346 Based on the discussions in the previous sections, the rest of 347 the paper is organized as follows: First, we study the cross-layer 348 aided routing design jointly considering both the PHY layer and 349 the NET layer [72], as shown in Section II; Then in Section III 350 we further investigate the cross-layer aided routing design 351 concept by jointly considering the PHY layer, the DL layer 352 and the NET layer [73], [74]. We commence by considering 353 Traditional Routing (TR) relying on a fixed transmit power 354 in Section III-A, while TR combined with Power Allocation 355 (PA) is discussed in Section III-B and Opportunistic Routing 356 (OR) using PA is studied in Section III-C; Finally, Section IV 357 concludes this treatise and offers some design guidelines. Fig. 5 358 lists the structure of this paper.

359 The notations used in this treatise are defined as follows:

$360-N$ : the number of nodes in the network;

361 - $H$ : the number of hops in an established route;

362 - $N_{r}$ : the maximum number of MAC retransmissions, in-

363 cluding the first transmission attempt;

$364-P_{t}$ : the transmit power of each node;

$365-P_{t_{i}}$ : the transmit power in the $i$-th node of the established route;

- FER $R_{i}$ : the FER of the $i$-th link in an established route;

- $p_{i}$ : the successful reception probability of the $i$-th link, where $p_{i}=1-F E R_{i}$;

- $E_{T}$ : the sum of the energy dissipated by all the nodes in the network, including the data packets and the control packets;

- $\bar{E}_{T}$ : the overall energy dissipation $E_{T}$ normalized by the number of bits received in the application layer of the destination;

- $E_{\text {total }}$ : the sum of the energy dissipated by the data packets during their transmission in the network;

- $\bar{E}_{\text {total }}$ : the total energy dissipation $E_{\text {total }}$ normalized by the end-to-end successful reception probability, which is the average energy consumption dissipated by the entire system during the successful delivery of a packet from the source to the destination;

- $R_{e 2 e}$ : the number of information bits successfully delivered to the destination per second.

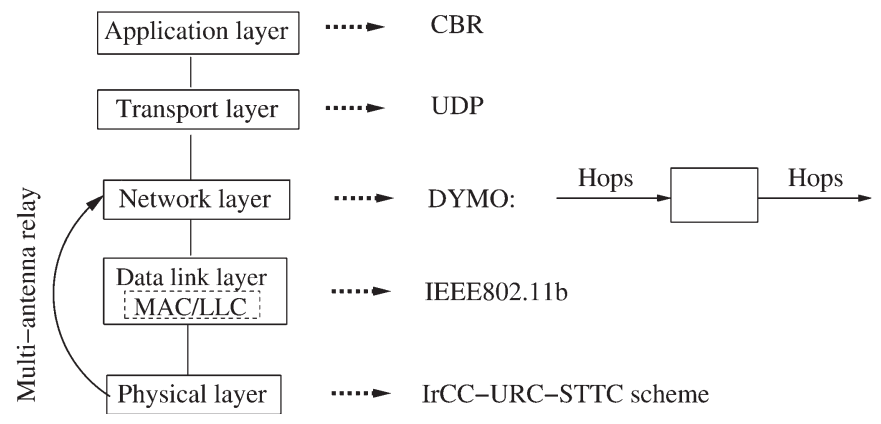

Fig. 6. System model of the energy-efficient routing with PHY \& NET cooperation in ad hoc networks.

\section{Routing Design With PHY \& NET Cooperation 384}

Energy-efficient wireless network design has recently at- 385 tracted wide-spread research attention [75]. Diverse error- 386 resilient Forward Error Correction (FEC) schemes were 387 proposed in [76] for achieving a low Bit Error Ratio (BER) at 388 near-capacity Signal-to-Noise Ratio (SNR) values. Therefore, 389 the effective transmission range can be improved, when the 390 required received signal power is reduced. Again, an Irregular 391 Convolutional Coded, Unity-Rate Coded and Space-Time Trel- 392 lis Coded (IrCC-URC-STTC) scheme has been proposed for 393 cooperative communications in [77]. Several Single-Antenna 394 RNs (SAs) were activated between the source and the destina- 395 tion. The RNs roaming closest to their optimal locations were 396 activated based on a technique relying on EXtrinsic Information 397 Transfer (EXIT) charts [78] in conjunction with near-capacity 398 code design principles, which were detailed for example in [79]. 399

However, the solution disseminated in [72] aims for minimiz- 400 ing the energy consumption by the joint design of both the PHY 401 and NET layers with the assistance of Multiple-Antenna Aided 402 Relay Nodes (MA-RN), as shown in the system model of Fig. 6. 403 Although the routing metric is still the number of hops, the em- 404 ployment of MA-RNs assists in reducing the potential number 405 of hops from the source to the destination, when dissipating a 406 given transmit power at each node. Therefore MA-RNs are ca- 407 pable of reducing the entire system's energy consumption. The 408 influence of the number of MA-RNs in a system will be studied 409 in Section II-C. Both the perfect capacity-achieving coding ab- 410 straction and a realistic near-capacity coding scheme, namely a 411 three-stage-concatenated IrCC-URC-STTC arrangement is em- 412 ployed in the PHY layer. The IEEE802.11b regime [7] is used 413 in the DL layer. In the NET layer, the more efficient DYMO 414 routing protocol [34] is employed, because the DYMO routing 415 protocol imposes a lower network control load and it is more 416 flexible in a high-mobility environment. However, the scenario 417 considered in [72] is a stationary scenario. The investigation of 418 high-mobility scenarios was set aside for its future study. The 419 User Data Protocol UDP [80] is employed in the transport layer 420 and CBR data streaming is used in the application layer. The 421 channel model employed is an Additive White Gaussian Noise 422 (AWGN) channel subjected to both inverse-second-power free- 423 space path loss and to uncorrelated Rayleigh fading.

\section{A. Near-Capacity Coding Schemes}

Each MA-RN is assumed to be equipped with two anten- 426 nas. If more than one MA-RN exist in the multi-hop ad hoc 427 


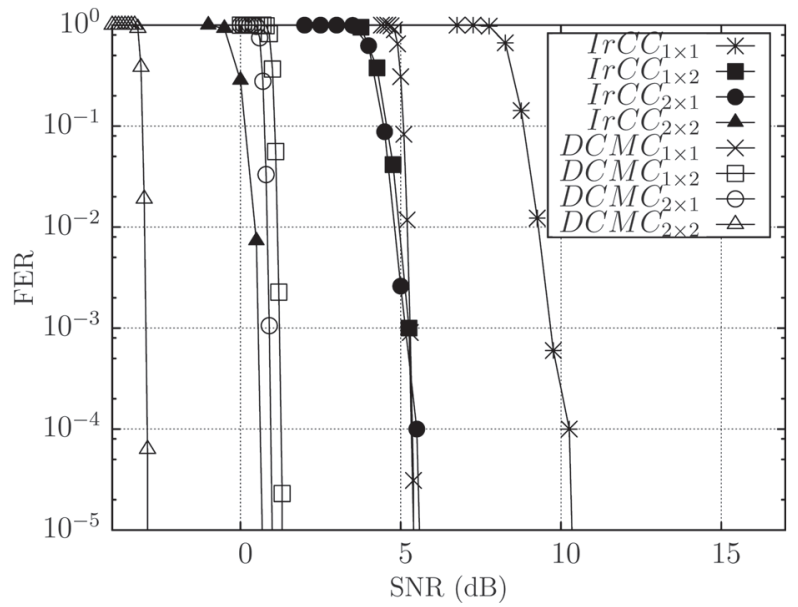

Fig. 7. FER performance of the four types of links, for example at the frame length of 1500 bits, of the uncorrelated Rayleigh fading channel for DCMCcapacity-based scheme and the IrCC-URC-STTC scheme, where $D C M C_{T \times R}$ represents the DCMC-capacity-based scheme, and $\operatorname{IrCC} T \times R$ represents the IrCC-URC-STTC scheme. Additionally, the subscript ' $T \times R$ ' represents having $T$ transmit antennas and $R$ receive antennas. Moreover, the overall FEC code rate is $R_{c}=0.5$, the effective throughput is $1 \mathrm{bps}$ (bits/symbol), the number of transmitted frames is 10000 and the IrCC has 17 component codes, associated with the weights $[0.049,0,0,0,0,0.24,0.16,0.12,0.035,0.102,0,0.071$, $0.093,0,0.091,0,0.039]$

428 network considered, then four different types of links may 429 appear. Specifically, there exists the SA-RN to SA-RN, SA$430 \mathrm{RN}$ to MA-RN, MA-RN to SA-RN and finally the MA-RN to 431 MA-RN links. All the MA-RNs employ the Quadrature Phase432 Shift Keying (QPSK)-assisted IrCC-URC-STTC scheme, while 433 all the SA-RNs employ the 8-ary Phase-Shift Keying (8PSK)434 assisted IrCC-URC scheme.

435 For example, the FER performance of all the four links at the 436 frame length of 1500 bits characterized by the Discrete-input 437 Continuous-output Memoryless Channel's (DCMC)-capacity 438 [81] and that of the IrCC-URC-STTC scheme is portrayed in 439 Fig. 7. It can be observed that the IrCC-URC-STTC scheme 440 performs close to the DCMC-capacity based scheme at a given $441 \mathrm{SNR}$ value. Meanwhile, the $\operatorname{IrCC}_{2 \times 2}$ scheme has a $5 \mathrm{~dB}$ gain 442 compared to $\operatorname{IrCC} C_{2 \times 1}$ or $\operatorname{IrCC} C_{1 \times 2}$ arrangements and has a nearly $44310 \mathrm{~dB}$ gain compared to the $\operatorname{IrCC_{1\times 1}}$ scheme at an FER of $44410^{-5}$, where $\operatorname{IrCC} C_{T \times R}$ represents the IrCC-URC-STTC scheme 445 and the subscript ' $T \times R$ ' indicates having $T$ transmit and $R$ 446 receive antennas. Hence, for the sake of guaranteeing the same 447 FER performance, $\operatorname{IrCC}_{2 \times 2}$ exhibits a larger transmit range at 448 a given transmit power and may hence potentially reduce the 449 number of hops required for conveying a message from the 450 source to the destination, which can be explained by analyzing 451 the calculation of the transmission range. More explicitly, the 452 average maximum transmission range is defined as the range, 453 over which the receiver node is capable of receiving a transmit454 ted packet with $F E R<10^{-5}$.

455 The required minimum signal-to-noise ratio $S N R_{d B}^{*}$ may be 456 calculated from the minimum receive power $P_{r}^{*}$ expressed in $457 \mathrm{dBm}$ as follows

$$
S N R_{d B}^{*}=10 \log _{10}\left(\frac{P_{r}^{*}}{N_{0}}\right)
$$

(a)

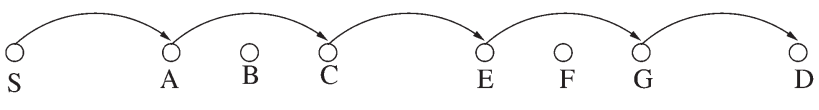

(b)

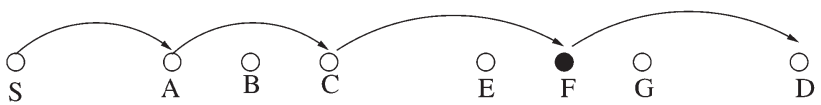

(c)

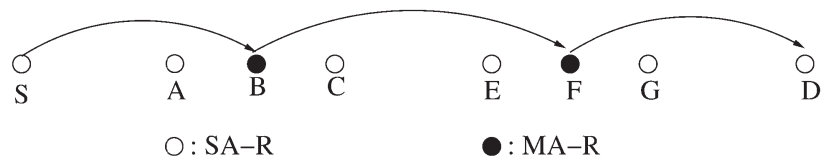

Fig. 8. The influence of MA-RNs on the routing strategy: (a) $H=5$ hops without MA-RNs; (b) $H=4$ with $1 \mathrm{MA}-\mathrm{RN}$ at point $F$; (c) $H=3$ with 2 MARNs at points $B$ and $F$.

where $N_{0}$ is the thermal noise power. Hence, given the transmit- 458 ted power $P_{t}$ and $S N R_{d B}^{*}$, the average maximum distance $d_{\max } 459$ from the transmitter, where the SNR requirement $S N R_{d B}^{*}$ may 460 'just' be satisfied to guarantee $F E R<10^{-5}$, is given by

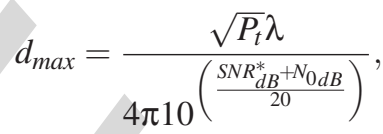

where the carrier's wavelength $\lambda=c / f$ and $N_{0 d B}=10 \log _{10} N_{0} .462$ $c$ is the speed of light in vacuum and $f$ is the carrier frequency. 463

Naturally, if the value of $P_{t}$ and $N_{0 d B}$ are fixed, then it may be 464 readily seen how the adequately 'illuminated' distance, where 465 the required target-FER may be maintained, will vary as a 466 function of the SNR value. As seen from Fig. 7, the maximum 467 adequately covered communication distance from MA-RN to 468 MA-RN is the highest, while that from SA-RN to SA-RN is the 469 lowest. Conversely, if $P_{t}$ and $d_{\max }$ are fixed, then the FER is the 470 lowest for the MA-RN to MA-RN link, while it is the highest 471 for the SA-RN to SA-RN link.

\section{B. Routing Algorithms}

It was shown in [77] that the IrCC-URC-STTC scheme is 474 capable of operating near the link's capacity, hence a substantial 475 power saving may be attained. When this scheme is employed 476 by the MA-RNs of the ad hoc network considered, the different 477 error correction capability of the four different types of links 478 will influence the routing strategy. Fig. 8 provides an example 479 on how the routing strategy is influenced.

As seen from Fig. 8, the network consists of $N=8$ nodes, 481 where $S$ is the source and $D$ is the destination. In Fig. 8(a), all 482 nodes are equipped with a single antenna, hence all links are 483 SA-RN to SA-RN links, which yields $H=5$ hops from $S$ to $D .484$ A single MA-RN is employed at point $F$ in Fig. 8(b), where the 485 packets arriving at node $C$ are directly transmitted to node $F .486$ Then, node $F$ will forward its received packets further to the 487 destination $D$. More specifically, the $C$-to- $F$ link is a SA-RN 488 to MA-RN link, while the $F$-to- $D$ link is an MA-RN to $D$ link, 489 where the $F-D$ distance is higher than that between the single- 490 antenna nodes of Fig. 8(a). Consequently, the number of hops 491 from $S$ to $D$ is decreased to $H=4$. In Fig. 8(c), two MA-RNs, 492 namely $B$ and $F$, are employed. The number of hops is further 493 decreased to $H=3$ as a benefit of using MA-RNs for nodes $B 494$ and $F$. 


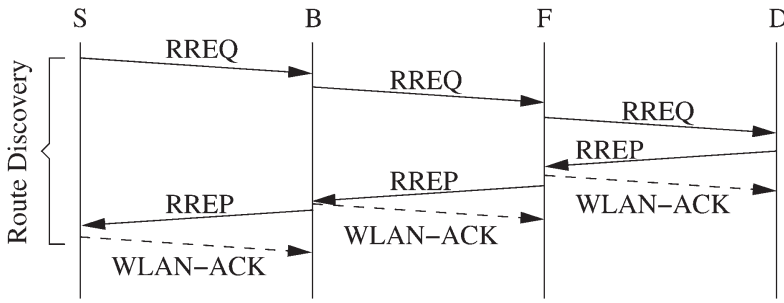

Fig. 9. The process of route discovery in the DYMO routing algorithm.

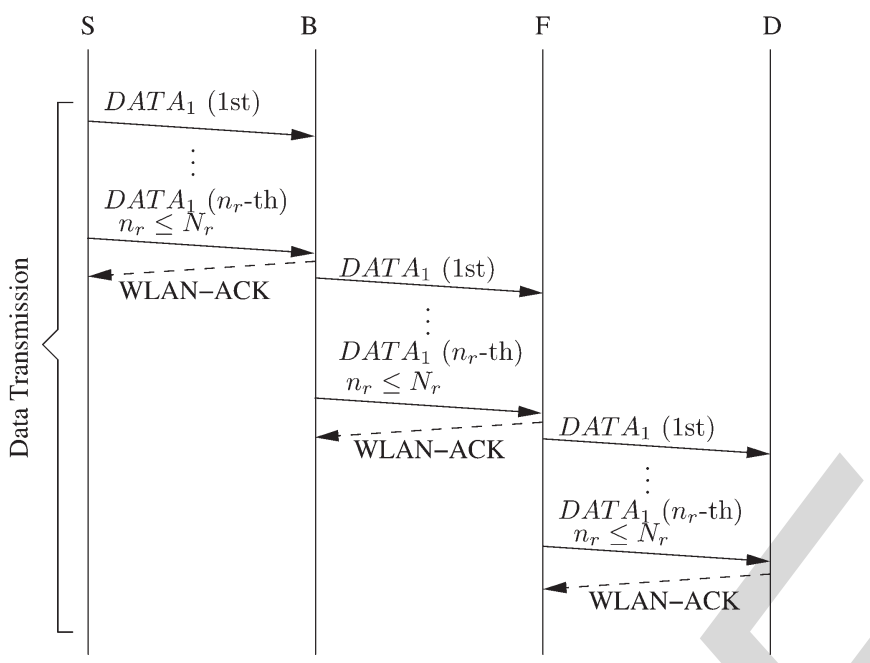

Fig. 10. The process of data transmission after a route is found from source $A$ to destination $D$.

496 The DYMO routing protocol is employed in the NET layer, 497 which combines most of the benefits of the AODV [33] and 498 DSR [32] protocols. The DYMO routing protocol always opts 499 for the specific route having the lowest number of hops to the 500 destination. When employing the MA-RN aided IrCC-URC501 STTC scheme, it will be demonstrated that the route selected 502 may be expected to have a further reduced number of hops. 503 The DYMO routing protocol is constituted by two main stages, 504 namely the route discovery and route maintenance. During the 505 route discovery, the Route REQuest (RREQ) and the Route 506 REPly (RREP) packets are used for identifying a route from 507 the source to the destination. By contrast, during the route 508 maintenance phase, a Route ERRor (RERR) packet is returned 509 to the source, when a broken link is detected. Figs. 9-11 show 510 the process of route discovery and data transmission as well 511 as route maintenance for the DYMO routing protocol, which 512 assisted us in analyzing the total energy consumption of the 513 system. The topology considered in Figs. 9-11 has a source $S$, 514 a destination $D$ and the pair of RNs $B$ and $F$. It is assumed that 515 each node is only capable of communicating with its neighbour 516 nodes. For example, node $B$ can only communicate with node $517 S$ and node $F$, while it cannot communicate with node $D$. The 518 exchange of the control packets between the neighbour nodes, 519 such as the exchange of the RREQ packet, RREP packet and 520 RERR packet, and the associated data transmission process is 521 detailed as follows:

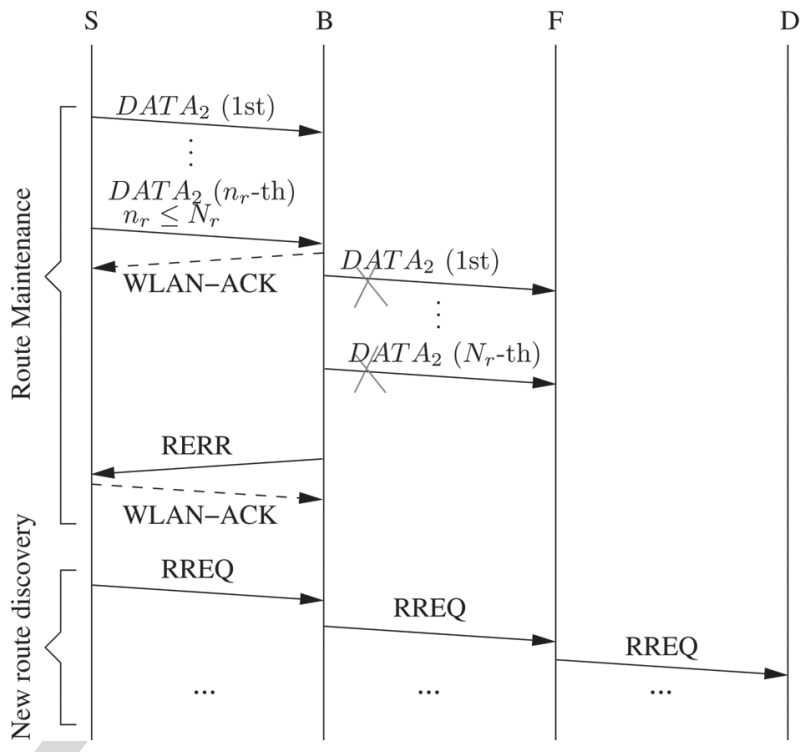

Fig. 11. The process of route maintenance in the DYMO routing algorithm.

As seen in Fig. 9, first the source $S$ broadcasts an RREQ 523 packet and when node $B$ receives this RREQ packet, it 524 broadcasts it. Then node $F$ receives the RREQ packet and 525 broadcasts it again. Finally, the destination $D$ receives the 526 RREQ packet, which originated from the source $S$; The 527 destination $D$ responds to the RREQ packet with a newly 528 generated RREP packet. The routing table of each node 529 is refreshed, when ever an RREQ/RREP packet arrives 530 at a node. Additionally, a Wireless Local Area Network- 531 Acknowledgement (WLAN-ACK) packet $^{1}$ is required for 532 confirming the successful reception of the RREP packet. 533

- Data Transmission process of Fig. 10. 534

When the RREP packet arrives at the source $S$ during 535 the process of route discovery, the source $S$ is informed 536 of a route spanning from the source $S$ to the destination 537 $D$, with node $B$ being the next hop of this route. Hence, 538 as seen in Fig. 10, the buffered data packet $D A T A_{1}$ is 539 transmitted to node $B$ according to the routing information 540 stored in the routing table of source $S$. If the packet $D A T A_{1} 541$ failed to reach node $B$, then node $B$ has to retransmit 542 the packet $D A T A_{1}$ until the number of retransmission 543 reaches its maximum of $N_{r}$. If and only if node $B$ receives 544 the packet $D A T A_{1}$ successfully within $n_{r}$ retransmissions, 545 where $n_{r} \leq N_{r}$, it would respond to source $S$ by sending 546 back a WLAN-ACK packet. The WLAN-ACK is used 547 for confirming the successful transmission of the packet 548 $D A T A_{1}$. Meanwhile, node $B$ forwards the packet $D A T A_{1}$ to 549 node $F$, since node $F$ is its next hop en route to destination 550 $D$. The routing information stored in node $B$ 's routing table 551 is obtained during the route discovery process as well. In 552 a similar way, if node $F$ successfully receives the packet 553 $D A T A_{1}$, it respond with a WLAN-ACK to node $B$ and 554

\footnotetext{
${ }^{1}$ The Acknowledgement packet is the one, which is returned to the transmitter as the acknowledgement of the correctly received data in the DL layer, hence it is referred to as WLAN-ACK in this treatise, where 'WLAN-ACK' represents the ACK packet employed in the IEEE802.11 standard. It is assumed that no Request-To-Send (RTS)/Clear-To-Send (CTS) mechanism is employed.
} 

586 Consequently, the sum of the energy $E_{T}$ dissipated by all nodes 587 in the network is given by

$$
\begin{aligned}
E_{T}= & \sum E_{\text {Route_Discovery }}+\sum E_{\text {Data_transmission }} \\
& +\sum E_{\text {Route_Maintenance }},
\end{aligned}
$$

588 where $E_{T}$ indicates the energy dissipated by a specific network 589 topology. $\sum E_{\text {Route_Discovery }}$ denotes the sum of energy dissi590 pated by the RREQ, the RREP and the WLAN-ACK packets 591 during the route discovery phase, which is shown in Fig. 9. 592 Furthermore, $\sum E_{\text {Route_Maintenance includes all the energy during }}$ 593 the route maintenance phase, except for $\sum E_{\text {Data_Transmission, }}$ 594 which is the energy dissipated by the data packets and by 595 the corresponding WLAN-ACK packets, as shown in Figs. 10 596 and 11.

\section{C. System Analysis}

598 The overall energy consumption $E_{T}$ of the entire network is 599 dependent on numerous parameters, such as the node density $600 \rho$, the number of MA-RNs $n_{M A}$, the mobile speed, the number 601 of hops $H$ of the selected route and the amount of bits $L_{a p p}$ 602 received in the application layer of the destination. To reduce 603 the dimensionality of the investigations when characterizing 604 the benefits of MA-RNs on the node's achievable transmission 605 range and FER performance, the node density $\rho$, the mobile 606 speed and $L_{a p p}$ are assumed to be constant, then $E_{T}$ is further 607 normalized by $L_{a p p}$ and $N$ of the entire network, where $N$ is re-

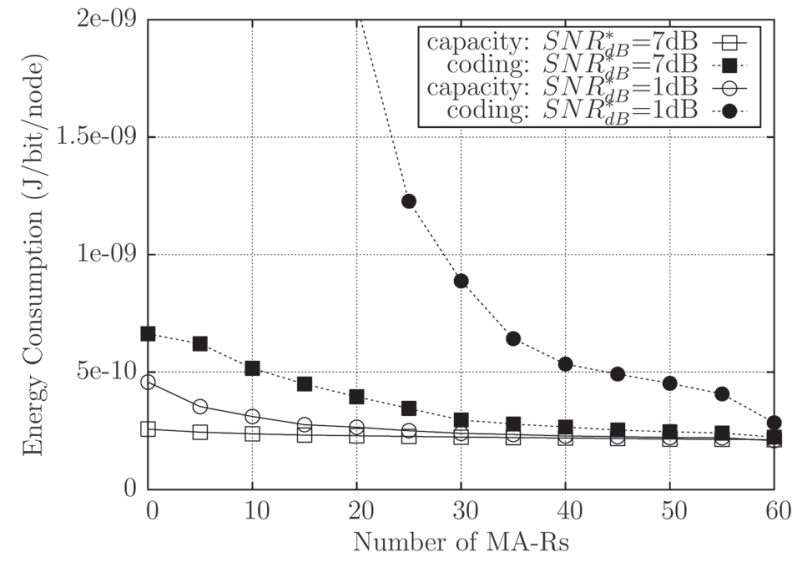

Fig. 12. Energy consumption $\bar{E}_{T}$ versus the number of MA-RNs $n_{M A}$ aiming for comparing the IrCC-URC-STTC scheme and the DCMC-capacity-based benchmark scheme at $S N R_{d B}^{*}$ of $7 \mathrm{~dB}$ and $1 \mathrm{~dB}$, where 'coding' denotes the IrCC-URC-STTC scheme and 'capacity' represents the DCMC-capacity-based benchmark scheme.

TABLE IV

SYSTEM PARAMETERS

\begin{tabular}{|l|r|}
\hline \hline Path-loss exponent & 2 \\
\hline Sensitivity threshold [82], $P_{r}^{*}$ & $-85 \mathrm{dBm}$ \\
\hline Mobility & stationary $(0 \mathrm{~m} / \mathrm{s})$ \\
\hline Simulation time & 30 second \\
\hline Number of simulation runs & 100 \\
\hline \hline
\end{tabular}

lated to the node density. Finally, the Normalized Energy Con- 608 sumption (NEC) $\bar{E}_{T}$ of the entire network can be expressed as: 609

$$
\bar{E}_{T}=\frac{E_{T}}{N L_{a p p}}=F\left(n_{M A}, H\right) .
$$

Four scenarios are considered to study the relationship be- 610 tween the number of MA-RNs and the energy consumption, 611 where $N=60$ stationary nodes are uniformly located in a 612 $500 \mathrm{~m} \times 500 \mathrm{~m}$ field, hence the node density is $\rho=240$ nodes 613 per square kilometer. The source $S$ and the destination $D$ are 614 located in the position $(499,499)$ and $(0,0)$, respectively. The 615 number of MA-RNs ${ }^{2}$ is increased from $n_{M A}=0$ to $n_{M A}=60616$ in steps of 5. The frame length of the data packets, which are 617 generated by the application layer, is $L_{a p p}=504$ Bytes. The 618 802.11b standard is employed in the DL layer. The transmit 619 power is set to $P_{T}=1 \mathrm{~mW}$. The other system parameters 620 employed for the simulations of Fig. 12 are listed in Table IV, 621 where the receiver's sensitivity [82] threshold is used to judge 622 whether the received signal is deemed to be noise, because a 623 received signal power below the sensitivity level is deemed to 624 be noise.

The energy consumption is quantified for both the IrCC- 626 URC-STTC scenario ${ }^{3}$ and the DCMC-capacity-based bench- 627 mark scenario at $S N R_{d B}^{*}$ of $7 \mathrm{~dB}$ and $1 \mathrm{~dB}$. As seen from 628 Fig. 12, the energy consumption of the IrCC-URC-STTC 629 scheme and of the benchmark scheme decrease upon increasing 630

\footnotetext{
${ }^{2}$ The number of MA-RNs also includes the source and the destination. Again, the multi-antenna aided nodes are denoted as MA-RNs and single-antenna nodes are denoted as SA-RNs.

${ }^{3}$ In the IrCC-URC-STTC scenario the network consists of QPSK-assisted IrCC-URC-STTC aided MA-RNs and 8PSK-assisted IrCC-URC aided SA-RNs.
} 
631 the number of MA-RNs $n_{M A}$. As mentioned in Section II-A, 632 a low FER and a relatively high transmission range i.e. cov633 erage area may be ensured by using the IrCC-URC-STTC 634 scheme advocated. Furthermore, as justified in Section II-B, 635 the specific routes having the lowest number of hops tend to be 636 activated in the MA-RNs aided network considered. Therefore, 637 having a high PHY-layer FER results in an increased number 638 of retransmissions and hence may trigger route re-discovery, 639 which results in more control packets being transmitted. Hence, 640 more energy per payload bit is required for successfully de641 livering the source data to the destination, as demonstrated 642 in Fig. 12.

\section{3 \\ 644 \\ III. Routing Design With PHY \& DL \& NET COOPERATION}

645 In recent years, numerous energy-efficient techniques have 646 been proposed [26], [64], [65], [69], [83]-[106]. However, 647 simply minimizing the energy consumption results in deficient 648 designs. It is more beneficial to strike a tradeoff between the 649 energy consumed and other metrics, such as the attainable 650 throughput. For example, Multiple-Input and Multiple-Output 651 (MIMO) schemes and near-capacity Space-Time Codes (STCs) 652 were employed in [84] for optimizing the RN selection for 653 the sake of maximizing the end-to-end throughput at a given 654 total available power. While single-hop transmissions are more 655 suitable for bandwidth-limited scenarios, multi-hop transmis656 sions combined with spatial frequency-reuse tend to perform 657 better in power-limited situations [69]. Spatial frequency-reuse 658 employed in multi-hop scenarios may be beneficially com659 bined with Interference Mitigation (IM) [69], [84] and transmit 660 beamforming [84] for the sake of finding an attractive balance 661 between energy minimization and throughput maximization in 662 both single-hop and multi-hop schemes [69], [85], [86]. As a 663 further advance, a beneficial tradeoff between the total energy 664 consumption and throughput was found in [85] by considering 665 both the transmission strategy of each node as well as the 666 location of the RNs and the data rate of each node.

667 Moreover, the authors of [26], [57], [86], [89], [92], [95]668 [98], [101]-[104], [107] invoked cross-layer design. For ex669 ample, the impact of the link error rate on the route selection 670 between a path associated with a large number of short-distance 671 hops and another with a smaller number of long-distance hops 672 was studied in [86]. In this paper, the link 'cost' was defined as 673 a function of both the energy required for a single transmission 674 attempt and the link error rate. This Objective Function (OF) 675 captures the cumulative energy expended in reliable data trans676 fer for both reliable and unreliable link layers. In [107], several 677 routing algorithms were proposed, which opted for the route 678 with minimum energy consumption in a mixed hop-by-hop and 679 end-to-end retransmission mode. In the end-to-end retransmis680 sion mode, a single unreliable link may require retransmissions 681 from the source, and hence may require more energy for suc682 cessfully delivering packets. Consequently, routing protocols 683 play an important role in saving energy. The authors of [57] 684 took into account both the energy consumed by data packets as 685 well as by control packets and MAC retransmissions, because 686 ignoring the energy consumption of exchanging control packets might underestimate the actual energy consumption and thus 687 may lead to inefficient designs. However, the energy OFs em- 688 ployed in [57], [86], [107] exploited the assumption of having 689 access to a potentially infinite number of MAC retransmissions, 690 which is unrealistic. The employment of the OF proposed in 691 [57], [86], [107] is feasible only when the affordable number of 692 MAC retransmissions is infinite, which is formulated as

$$
E_{\text {total }}=\sum_{1}^{H} \frac{E_{i}}{1-F E R_{i}}
$$

where $\frac{1}{1-F E R_{i}}$ is the expected number of transmission at- 694 tempts required for successfully delivering a packet across 695 link $i$. As seen from (5), the total energy of all hops is simply 696 summed, which suggests that the success of the individual links 697 in a route is deemed to be independent of each other, since the 698 assumption that an infinite number of MAC retransmissions 699 is affordable is given. Additionally, although the authors of 700 [89] considered a limited number of MAC retransmissions, no 701 specific OF was formulated.

702

Furthermore, TR relies on a route discovery process invoked 703 for gleaning sufficient routing information for the source to 704 make meritorious routing decisions, regardless, whether the 705 routing protocol is proactive or reactive [108]. However, due to 706 the rapid fluctuation of the channel conditions, the routing in- 707 formation estimated on the basis of the average Channel Quality 708 Information (CQI) may become stale, resulting in suboptimum 709 routing. Therefore, OR [90]-[92], [96], [101], [103], [109]- 710 [114] has been proposed for avoiding this problem. In OR no 711 pre-selected route is employed, instead a so-called forwarder 712 $\mathrm{RN}$ set is used for forwarding the packets along a benefi- 713 cial route. The near-instantaneously varying characteristics of 714 wireless channels is beneficially exploited considered by OR. 715 Table V shows that OR is widely used in various networks, such 716 as ad hoc networks [103], [115], wireless sensor networks [91], 717 cognitive networks [116], vehicular networks [117], [118] and 718 DTNs [119]-[121].

More specifically, Liu et al. [110] illustrated the basic idea 720 behind OR and categorized the potential design criteria, includ- 721 ing the Estimated Transmission count (ETX), the geographic 722 distance aided and the energy consumption based philosophies. 723 Biswas and Morris [101] proposed an Extremely Opportunistic 724 Routing (ExOR) scheme, which employed the ETX metric at 725 the destination for deciding the priority order of selecting a 726 $\mathrm{RN}$ from the potential forwarder set. The proposed routing 727 regime integrated the routing protocol and the MAC protocol 728 for the sake of increasing the attainable throughput of multi- 729 hop wireless networks. Their solution [101] also exploited 730 the less reliable long-distance links, which would have been 731 ignored by traditional routing protocols. Moreover, Dubois- 732 Ferrière et al. [111] conceived the Least-Cost Anypath Routing 733 (LCAR) regime, which finds the optimal choice of candidate 734 RNs relying on the expected ETX cost of forwarding a packet 735 to the destination. This LCAR algorithm considers the coordi- 736 nation of the link layer protocols. Laufer et al. [114] proposed 737 a 'polynomial-time multirate anypath' routing algorithm and 738 provided the proof of its optimality. The proposed routing 739 algorithm employed the Expected Anypath Transmission Time 740 
TABLE V

OpPortunistic Routing Protocols in VARIOUs Networks

\begin{tabular}{|c|c|c|}
\hline Year & Authors & Contribution \\
\hline 2006 & Pelusi et al. [117] & $\begin{array}{l}\text { Surveyed the most promising OR solutions and the taxonomy of the main routing and forwarding } \\
\text { approaches in challenging environments. }\end{array}$ \\
\hline 2008 & Conan et al. [121] & Proposed a single copy and multi-hop OR scheme for sparse Delay Tolerant Networks (DTNs). \\
\hline \multirow[t]{2}{*}{2009} & Khalife et al. [118] & $\begin{array}{l}\text { Explored opportunistic forwarding without preestablished routing in multihop cognitive radio } \\
\text { networks. }\end{array}$ \\
\hline & Spyropoulos et al. [122] & $\begin{array}{l}\text { Proposed a class of routing schemes that can identify the nodes of "highest utility" in intermittently } \\
\text { connected wireless networks. }\end{array}$ \\
\hline \multirow[t]{2}{*}{2010} & Lee et al. [119] & $\begin{array}{l}\text { Presented a topology-assisted Geo-OR designed for vehicular networks that combined topology- } \\
\text { assisted geographic routing with opportunistic forwarding for mitigating the effects of unreliable } \\
\text { wireless channels. }\end{array}$ \\
\hline & Li et al. [123] & $\begin{array}{l}\text { Investigated energy-efficient opportunistic forwarding and designed different forwarding policies for } \\
\text { DTNs. }\end{array}$ \\
\hline 2011 & Mao et al. [91] & $\begin{array}{l}\text { Presented an energy-efficient OR strategy conceived for wireless sensor networks, which created a } \\
\text { prioritized forwarder list to minimize the total energy consumption of all nodes. }\end{array}$ \\
\hline 2012 & Wang et al. [103] & $\begin{array}{l}\text { Proposed a cooperative OR scheme for mobile ad hoc networks for tackling the problem of } \\
\text { opportunistic data transfer. }\end{array}$ \\
\hline 2013 & Wu et al. [120] & $\begin{array}{l}\text { Proposed a hybrid routing scheme for data dissemination in vehicular ad hoc networks (VANETs), } \\
\text { using a carry-and-forward scheme for mitigating the forwarding disconnection } \\
\text { problem of sparse VANETs. }\end{array}$ \\
\hline 2014 & Yoon et al. [124] & $\begin{array}{l}\text { Investigated the feasibility of OR in power line communications access networks and proposed a } \\
\text { customized OR, which used static geographical information. }\end{array}$ \\
\hline
\end{tabular}

741 (EATT) as the routing metric, which is a generalization of the 742 unidirectional ETX metric that takes into account that nodes 743 transmit at multiple bit rates. The authors of [90], [109], [113] 744 employed a geographic distance based metric for choosing the 745 potential forwarder RN set. More specifically, Zorzi and Rao 746 [109] proposed an OR scheme based on random forwarding, 747 where the specific node, which is closest to the destination 748 is chosen as the RN for the next hop. Additionally, they [90] 749 analyzed the achievable energy as well as latency performance 750 and provided a detailed description of a MAC scheme based 751 on both opportunistic concepts and on collision avoidance. 752 Zeng et al. [113] proposed a multirate OR by incorporating 753 rate adaptation into their candidate-selection algorithm, which 754 was shown to achieve a higher throughput and lower delay 755 than the corresponding traditional single-rate routing and its 756 opportunistic single-rate routing counterpart. The authors of 757 [91], [92], [96] employed the energy consumption metric for 758 choosing the potential forwarder RN set. More concretely, 759 Mao et al. [91] presented an energy-efficient OR strategy 760 relying on sophisticated PA, which prioritizes the forwarder 761 RNs by directly minimizing the total energy consumption of 762 all nodes. Dehghan et al. [92] developed a minimum-energy 763 cooperative routing based on many-to-many cooperation and 764 determines the optimal route with the aid of the Bellman-Ford 765 algorithm [123]. Wei et al. [96] proposed an energy-conserving 766 Assistant Opportunistic Routing (AsOR) protocol, which clas767 sified a sequence of nodes into three different node sets, namely, 768 the frame node, the assistant node and the unselected node. 769 The frame nodes were indispensable for decode-and-forward 770 operation, while the assistant nodes provided protection against 771 unsuccessful opportunistic transmissions. Although the authors 772 of [91], [92], [96] employed the energy consumption as their 773 routing metric, they have not provided any theoretical bounds 774 in their performance analysis. Moreover, these authors as775 sumed that the number of affordable MAC retransmissions 776 was infinite.

777 An appropriate PA scheme combined with an opportunistic 778 scheme was introduced in [74]. The opportunistic scheme does

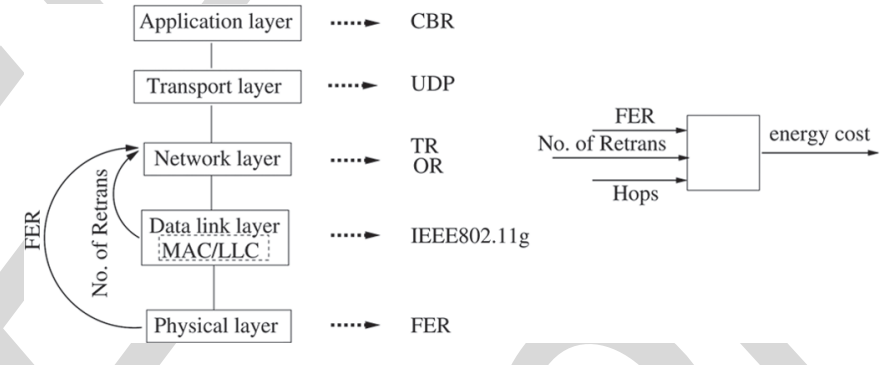

Fig. 13. System model of the energy-efficient routing with PHY \& DL \& NET cooperation in ad hoc networks.

not employ a pre-selected route, while it will fully utilize 779 the time-variant characteristic of the hostile wireless channel, 780 where any RN has the chance to forward a packet as long as 781 the packet arrives at this RN successfully. A pair of energy- 782 consumption-based OFs are constructed for TR and OR by 783 exploiting the knowledge of both the corresponding FER within 784 the PHY layer, as well as that of the number of MAC retrans- 785 missions and of the number of RNs in the NET layer, as seen 786 in the system model of Fig. 13. The above-mentioned TR and 787 OR algorithms employ the corresponding energy-consumption- 788 based OFs as their routing metrics, respectively. Apart from 789 the energy consumption, the end-to-end throughput is evaluated 790 as well. It was demonstrated that the algorithms proposed in 791 [74] are capable of operating close to the theoretical bound 792 found by the exhaustive search of all routes. In Fig. 13, the 793 characteristics of the PHY layer are represented with the aid 794 of the FER, while the DL layer employs the IEEE802.11g 795 standard. In the NET layer, the above-mentioned TR and OR 796 are employed, which make their decisions on the basis of the 797 above energy-consumption-related OFs. The UDP is employed 798 in the transport layer and the data streaming relies on a CBR 799 service in the application layer. As in Section II, the channel 800 imposes both free-space path-loss and uncorrelated Rayleigh 801 fading, plus the ubiquitous AWGN.

802

Based on the system model of Fig. 13, the impact of the 803 lowest three layers of the OSI model on the total energy 804 
805 dissipated of the entire system is considered, which will be 806 analyzed, whilst relying on an energy-consumption-based OF. 807 In [73], [74], only the transmit energy consumed by the 808 data packets during their transmission is considered, which are 809 generated by the application layer. The energy consumed by 810 other packets, such as routing and MAC control packets is not 811 considered. In other words, the idealized simplifying assump812 tion is that the energy consumed during the process of route 813 discovery is negligible. The elimination of this simplification 814 was set aside for the future work. As detailed in [73], [74], 815 the $\mathbf{O F}$ is invoked for making routing-related decisions, which 816 directly influence the energy consumed by future data packets. 817 All nodes are assumed to be stationary. Only a single source818 destination pair is supported in the network and only a single 819 node has the chance of transmitting in a time slot, once the route 820 was determined. All the data packets are also assumed to have 821 the same length and all nodes have the same transmission rate.

\section{A. Traditional Routing With Fixed Transmit Power}

823 Naturally, having an infinite number of MAC retransmis824 sions will impose a potentially infinite end-to-end delay at the 825 destination, which is not realistic. In realistic environments, 826 the wireless link may become broken owing to packet errors 827 if the maximum number of MAC retransmissions has been 828 exhausted. A broken link may trigger a route-repair or even 829 route re-discovery for the sake of maintaining the current 830 source-destination communications session. The route-repair 831 is often required at the upper-node's broken link, while the 832 route re-discovery should be initiated by the source. All these 833 actions may consume more energy and naturally they reduce 834 the attainable throughput. Additionally, the success of a specific 835 hop emanating from a node relies on the success of all previous 836 hops. If any of the previous links is broken, then no packet 837 will be forwarded towards the destination. Naturally, any link 838 is more likely to break if the number of MAC retransmissions 839 is limited to $N_{r}$. The energy consumption considered is divided 840 into two parts: the energy consumed by the data packets which 841 succeed in reaching the destination and the energy consumed 842 by the data packets which are dropped before reaching the 843 destination. The time slot duration of a single transmission 844 attempt across a given link is defined as $T$. Given the same data 845 packet length and the same transmission rate at each node, $T$ is 846 a constant value. Here, the energy-conscious OF of a two-hop 847 route is detailed as an example. $p_{s}$ and $p_{f}$ are used to denote 848 the probability of a packet being successfully delivered to the 849 destination successfully and being dropped before reaching the 850 destination, respectively. Furthermore, the notation $p_{s}(\tau)$ repre851 sents the probability that the packet is successfully delivered 852 all the way from the source to the destination after a time 853 duration of $\tau$. First, the energy consumption analysis of a 2-hop 854 route is considered in Fig. 14. The symbol $\sqrt{ }$ indicates that 855 the link's transmission is successful after $1 \leq \frac{\tau}{T} \leq N_{r}$ MAC 856 retransmission attempts. Hence the time duration of the link's 857 transmission is $T \leq \tau \leq N_{r} T$. Fig. 14 shows that a packet's 858 successful transmission over the link $S-R_{1}$ requires a time 859 duration of $\tau_{1}$, while the successful transmission of a packet 860 over the link $R_{1}-D$ requires a time duration of $\tau_{2}$. Hence the

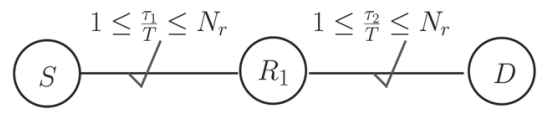

Fig. 14. A packet is successfully delivered from $S$ to $D$ in a 2-hop route.

(a)
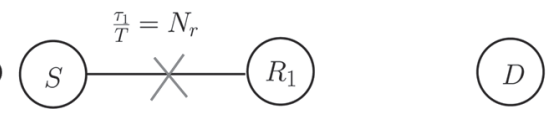

(b)

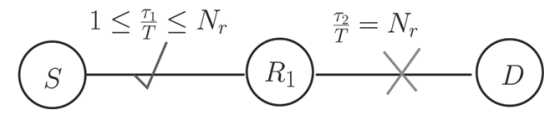

Fig. 15. A packet is dropped before reaching $D$ in a 2-hop route.

total time duration of a packet's passage between $S$ and $D$ is 861 $\left(\tau_{1}+\tau_{2}\right)$, where $2 T \leq \tau_{1}+\tau_{2} \leq 2 N_{r} T$.

Therefore,

$$
\begin{aligned}
p_{s}(2 T)= & p_{1} p_{2}, \\
p_{s}(3 T)= & \left(1-p_{1}\right) p_{1} p_{2}+p_{1}\left(1-p_{2}\right) p_{2}, \\
p_{s}(4 T)= & \left(1-p_{1}\right)^{2} p_{1} p_{2} \\
& +\left(1-p_{1}\right) p_{1}\left(1-p_{2}\right) p_{2}+p_{1}\left(1-p_{2}\right)^{2} p_{2}, \\
& \vdots \\
p_{s}\left(2 N_{r} T\right)= & \left(1-p_{1}\right)^{N_{r}-1} p_{1}\left(1-p_{2}\right)^{N_{r}-1} p_{2} .
\end{aligned}
$$

While $p_{s}$ is given by

$$
\begin{aligned}
p_{s} & =p_{s}(2 T)+p_{s}(3 T)+p_{s}(4 T)+\cdots+p_{s}\left(2 N_{r} T\right), \\
& =\sum_{1}^{N_{r}} \sum_{1}^{N_{r}}\left(1-p_{1}\right)^{i_{1}-1} p_{1}\left(1-p_{2}\right)^{i_{2}-1} p_{2} .
\end{aligned}
$$

Since during a single time slot $T$ the nodes consume an energy 865 of $E$, the estimated total energy $E_{s}$ consumed by a successfully 866 delivered packet in a two-hop route is

$$
E_{s}=\left[2 p_{s}(2 T)+3 p_{s}(3 T)+4 p_{s}(4 T)+\cdots+2 N_{r} p_{s}\left(2 N_{r} T\right)\right] E .
$$

In a similar way, the time $D_{s}$ required for a packet, which is 868 successfully delivered from $S$ to $D$ is given by

$$
D_{s}=\left[2 p_{s}(2 T)+3 p_{s}(3 T)+4 p_{s}(4 T)+\cdots+2 N_{r} p_{s}\left(2 N_{r} T\right)\right] T .
$$

Additionally, the packets, which exhausted the maximum 870 number $N_{r}$ of MAC retransmissions and were finally dropped 871 before reaching $D$ due to poor channel conditions also consume 872 energy. This energy should also be taken into account in the 873 total energy consumption. The energy dissipation analysis of a 874 packet dropped before reaching the destination in a 2-hop route 875 is portrayed in Fig. 15. The symbol $\times$ indicates that the link's 876 transmission fails after $\frac{\tau}{T}=N_{r}$ MAC retransmission attempts. 877 As seen in Fig. 15, a transmission failure may occur either in 878 the $S-R_{1}$ link or in the $R_{1}-D$ link of a 2-hop route. Hence, 879 even when the data transmission in the $S-R_{1}$ link is successful 880 within the time duration of $T \leq \tau_{1} \leq N_{r} T$, the transmission 881 might fail in the $R_{1}-D$ link. 


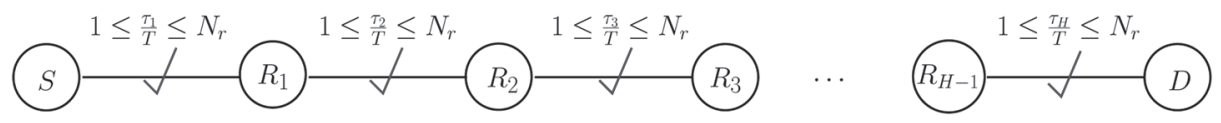

Fig. 16. A packet is successfully delivered from $S$ to $D$ in a $H$-hop route.

(a)

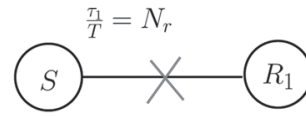

(b)

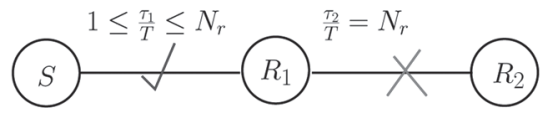

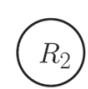
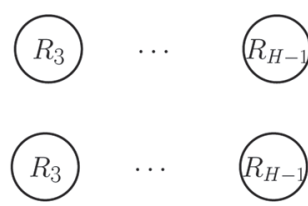

$\cdots$
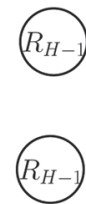

(c)
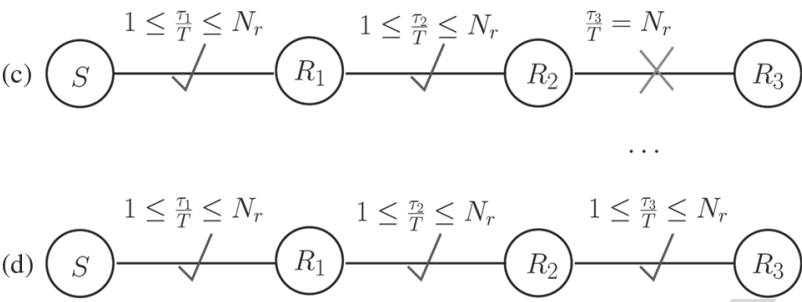

$1 \leq \frac{\tau_{3}}{T} \leq N_{r}$
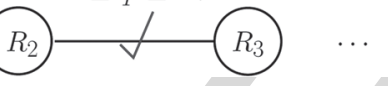

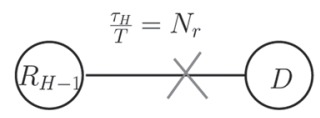

Fig. 17. A packet is dropped before reaching $D$ in a H-hop route.

883 The probability of failure $p_{f}$ of the two-hop route for a single 884 packet is described as follows:

$$
\begin{aligned}
p_{f}(1) & =\left(1-p_{1}\right)^{N_{r}}, \\
p_{f}(2) & =\left[\left(1-p_{f}(1)\right)\right]\left(1-p_{2}\right)^{N_{r}}, \\
p_{f} & =p_{f}(1)+p_{f}(2),
\end{aligned}
$$

885 where $p_{f}(h)$ represents the probability of the packet becoming 886 dropped during the $h$-th hop. Therefore, the energy $E_{f}$ con887 sumed by a dropped packet is quantified as follows:

$$
E_{f}=\left[N_{r} p_{f}(1)+\sum_{1}^{N_{r}}\left(1-p_{1}\right)^{i_{1}-1} p_{1}\left(1-p_{2}\right)^{N_{r}}\left(i_{1}+N_{r}\right)\right] E .
$$

888 Similarly, the average time $D_{f}$ required by a packet to propagate 889 from $S$ up to the broken link is formulated as

$$
D_{f}=\left[N_{r} p_{f}(1)+\sum_{1}^{N_{r}}\left(1-p_{1}\right)^{i_{1}-1} p_{1}\left(1-p_{2}\right)^{N_{r}}\left(i_{1}+N_{r}\right)\right] T .
$$

890 The energy dissipation analysis of a packet's successful 891 delivery to the destination and that of a packet dropped before 892 reaching the destination in a $H$-hop route is characterized in 893 Fig. 16 and Fig. 17, respectively. Fig. 16 portrays the scenario, 894 where each link's transmission is successful after $1 \leq \frac{\tau}{T} \leq N_{r}$ 895 MAC retransmission attempts. By contrast, Fig. 17 shows that 896 a transmission failure could take place within any of the links, 897 where all the previous links' transmissions were successful. The 898 time duration elapsed before reaching the failed link is $\tau=N_{r} T$, 899 while that elapsing during all the previous link's transmission is $900 T \leq \tau \leq N_{r} T$.

901 Therefore, the total normalized transmit energy consumption 902 becomes:

$$
\bar{E}_{\text {total }}=\frac{E_{\text {total }}}{p_{s}}=\frac{E_{s}+E_{f}}{p_{s}} .
$$

Similarly, the end-to-end throughput $R_{e 2 e}$ is given as

$$
R_{e 2 e}=\frac{p_{s}}{D_{s}+D_{f}}
$$

A low-complexity routing algorithm is proposed in [73]. 904 The process of route discovery is shown in Fig. 18, where 905 $S$ represents the source, $D$ represents the destination, and the 906 other nodes are denoted by symbols $A, B, C, E, F$ and $G$. $E_{S \rightarrow n . t} 907$ denotes the estimated NEC for the route spanning from $S$ to 908 node $n$ at time instant $t$, while $E_{S \rightarrow n}$ is used for storing the 909 minimum NEC for every node in every time-slot of duration 910 $T$. The routing process may be divided into the following 911 four steps:

- Step 1 Node $S$ broadcasts the RREQ packet;

Step 2 Every node carries out the operations detailed in 914 Fig. 19 upon receiving the RREQ packet;

915

- Step 3 Node $S$ receives the RREP packet and then updates 916 the routing table;

- Step 4 Then node $S$ sends its data packet along the specific 918 route having the lowest estimated $\bar{E}_{\text {total }}$.

919

A flow chart is provided in Fig. 19 for specifically highlight- 920 ing the operations, when each node receives an RREQ packet. 921 If $S$ receives the RREQ packet, $S$ will simply discard this RREQ 922 packet. By contrast, if another node $n(n \neq S)$ receives the 923 RREQ packet, it calculates the NEC $E_{S \rightarrow n, t}$ and then compares 924 $E_{S \rightarrow n, t}$ to $E_{S \rightarrow n}$. If $E_{S \rightarrow n, t}>E_{S \rightarrow n}$, then node $n$ will discard the 925 RREQ packet. Otherwise, if node $n$ is $D$, then $D$ will respond 926 with a newly created RREP packet. However, if node $n$ is not 927 $D$, node $n$ will broadcast the RREQ packet again.

Now the process of routing discovery is explained in details 929 for further clarification. During time slot 1, node $S$ broadcasts 930 the RREQ packet, nodes $A, B$ and $C$ receive the RREQ packet. 931 According to the actions seen in Fig. 19, nodes $A, B$ and $C$ first 932 calculate $E_{S \rightarrow A, 1}, E_{S \rightarrow B, 1}$ and $E_{S \rightarrow C, 1}$, respectively. Then they 933 compare these newly calculated values with the aid of $E_{S \rightarrow A}, 934$ 
(a)

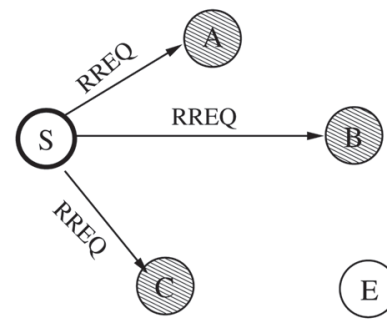

(c)
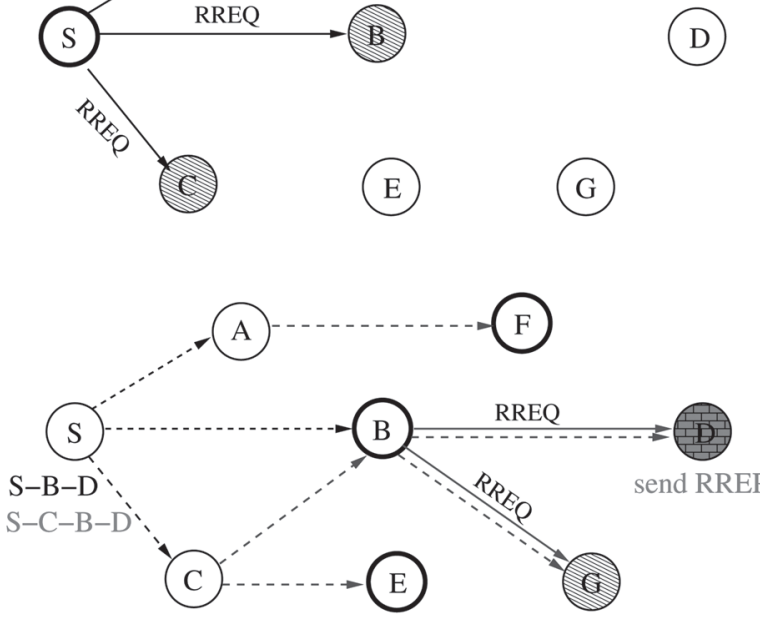

(d)

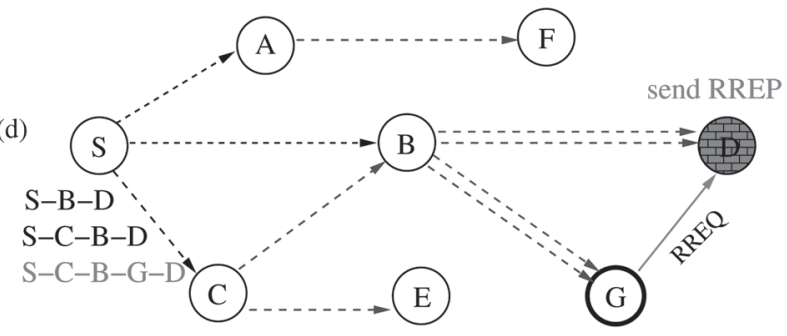

(b)

broadcast RREQ

ready to forward RREQ

ready to send RREP

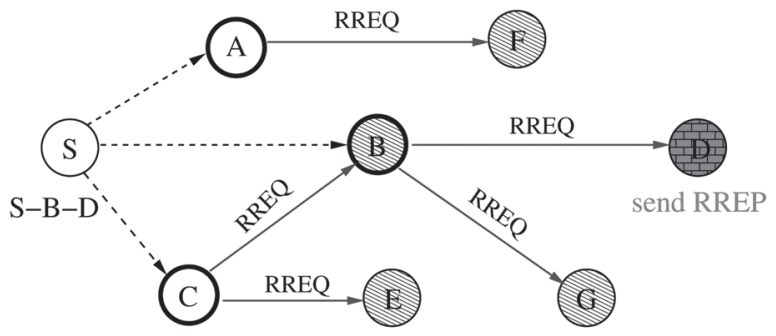

$\longrightarrow E_{S \rightarrow n, t \leq E_{S \rightarrow n}}$ at current transmission - - $-E_{S \rightarrow n, t \leq E_{S \rightarrow n}}$ at previous transmission

Fig. 18. The process of route discovery in the low-complexity routing algorithm. (a) Actions during time slot 1. (b) Actions during time slot 2 and node $S$ updates its routing table with the route $S-B-D$. (c) Actions during time slot 3 and node $S$ updates its routing table with the route $S-C-B-D$ since the estimated NEC of route $S-C-B-D$ is lower than that of route $S-B-D$. (d) Actions during time slot 4 and node $S$ updates its routing table with the route $S-C-B-G-D$, since the estimated NEC of route $S-C-B-G-D$ is lower than that of route $S-C-B-D$.

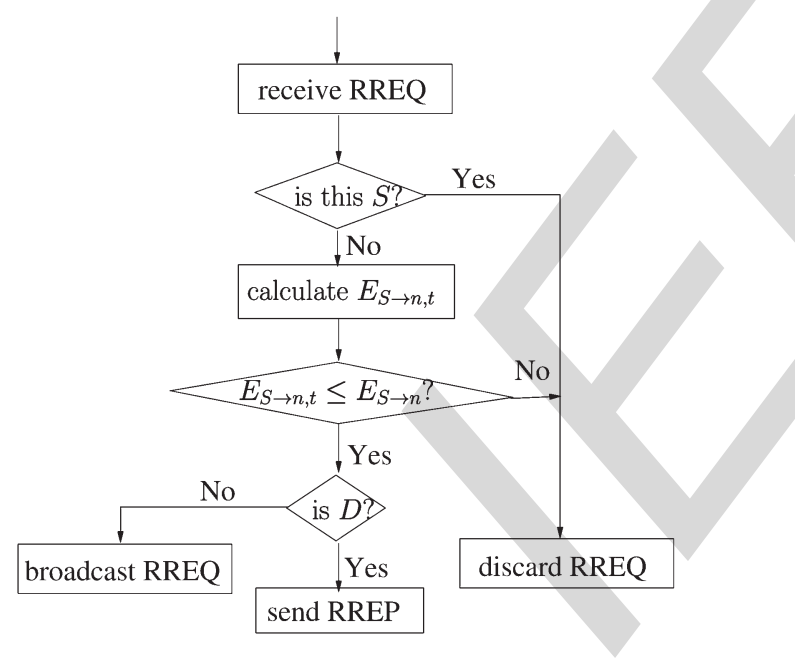

Fig. 19. Operations when each node receives an RREQ packet.

$935 E_{S \rightarrow B}$ and $E_{S \rightarrow A}$, respectively. If $E_{S \rightarrow A, 1} \leq E_{S \rightarrow A}$, then $A$ will 936 update $E_{S \rightarrow A}$ and will forward the RREQ packet during the 937 next time slot, otherwise it will discard it. The same actions 938 are carried out at node $B$ and node $C$ as well.

939 Then during time slot 2, node $A, B$ and $C$ forward the RREQ 940 packet. According to the actions seen in Fig. 19, nodes $B, E, F$ 941 and $G$ will forward the RREQ packet in the next time slot, since $942 E_{S \rightarrow B, 2} \leq E_{S \rightarrow B}, E_{S \rightarrow E, 2} \leq E_{S \rightarrow E}, E_{S \rightarrow F, 2} \leq E_{S \rightarrow F}$ and $E_{S \rightarrow G, 2} \leq$ $943 E_{S \rightarrow G}$. Node $D$ is ready to send back a newly created RREP 944 packet in the next time slot, since node $D$ is the destination and $945 E_{S \rightarrow D, 2} \leq E_{S \rightarrow D}$. Afterwards, $S$ will receive the RREP packet 946 and updates its routing table with the route $S-B-D$ obtained. $947 S$ will send the data packet along the route $S-B-D$.

948 During time slot 3, nodes $B, E, F$ and $G$ forward the RREQ 949 packet. According to the actions portrayed in Fig. 19, node $G$ 950 will forward the RREQ packet during the next time slot, since
$E_{S \rightarrow G, 3} \leq E_{S \rightarrow G}$. Node $D$ will then send back a newly created 951 RREP packet during the next time slot, since node $D$ is the 952 destination and $E_{S \rightarrow D, 3} \leq E_{S \rightarrow D}$. Afterwards, $S$ will receive the 953 second RREP packet and updates its routing table again with 954 the route $S-C-B-D$ obtained, since the estimated NEC of 955 route $S-C-B-D$ is lower than that of route $S-B-D$. Finally, 956 node $S$ will send the next data packet along the updated route 957 $S-C-B-D$.

958

During time slot 4 , node $G$ forwards the RREQ packet, 959 then nodes $B, E$ and $D$ receive it. According to the actions 960 of Fig. 19, then $D$ is ready to send back a newly created 961 RREP packet during the next time slot, since node $D$ is the 962 destination and $E_{S \rightarrow D, 4} \leq E_{S \rightarrow D}$. Afterwards, $S$ will receive the 963 third RREP packet and updates its routing table again with the 964 route $S-C-B-G-D$ obtained, since the estimated NEC of 965 route $S-C-B-G-D$ is lower than that of route $S-C-B-D .966$ Finally, node $S$ will send the next data packet along the updated 967 route $S-C-B-G-D$.

The analytically estimated NEC associated both with an 969 infinite number as well as a finite number of $N_{r}$ MAC re- 970 transmissions was calculated from (5) and (19), respectively. 971 A simple linear network topology was studied, where all $N 972$ nodes are equi-spaced along a line. The frame length of the 973 data packets, which are generated from the application layer, 974 is Lapp $=1024$ Bytes. The $802.11 \mathrm{~g}$ standard is employed in the 975 DL layer. The transmit power is set to $P_{t_{i}}=0.016 \mathrm{~mW}$ and the 976 IrCC-URC-QPSK defined in Section II is employed in the PHY 977 layer. The channel model is the AWGN channel subjected to 978 inverse second-power free-space path loss. The other system 979 parameters employed for the simulations of Figs. 20 and 21 are 980 listed in Table IV.

The NEC and the end-to-end throughput evaluated both from 982 (19) and (20) as well as by simulations are portrayed in Figs. 20983 and 21 . 


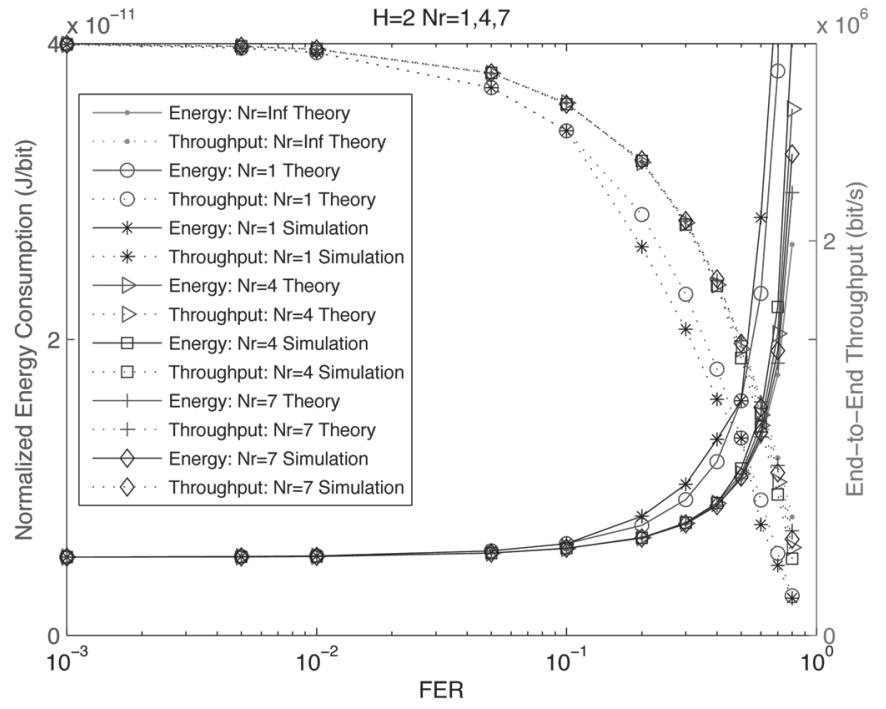

Fig. 20. The NEC and the end-to-end throughput versus FER $\left(10^{-3} \leq F E R \leq\right.$ 1) and the maximum number of MAC retransmissions $N_{r}\left(N_{r}=1,4,7\right)$ with the number of hops $H=2$ for comparing the theoretically analyzed values and simulation based values.

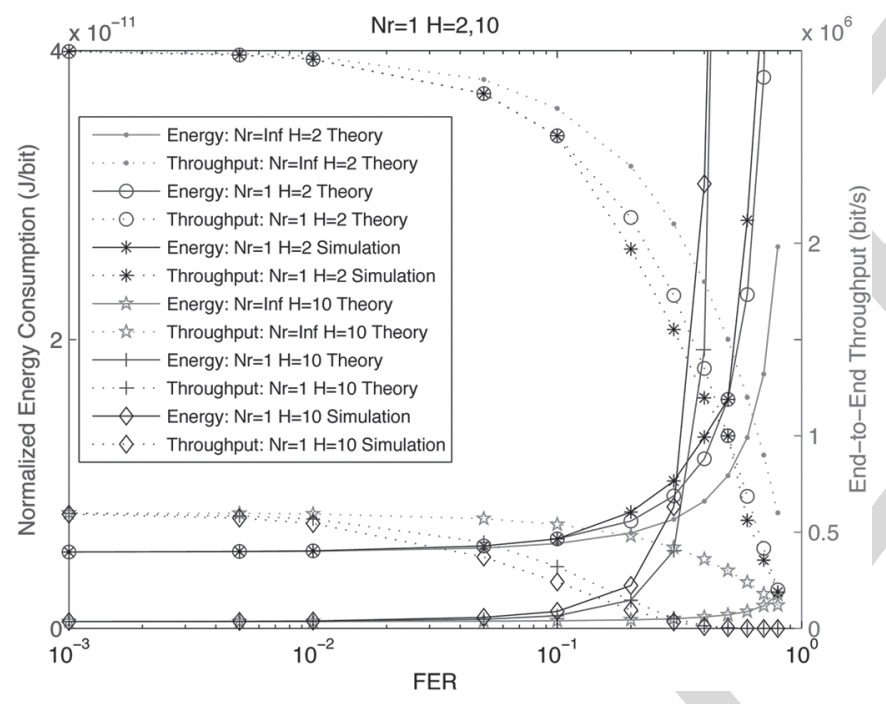

Fig. 21. The NEC and the end-to-end throughput versus FER $\left(10^{-3} \leq F E R \leq\right.$ 1) and the number of hops $H(H=2,10)$ with the maximum number of MAC retransmissions $N_{r}=1$ for comparing the theoretically analyzed values and simulation based values.

985 Fig. 20 displays three groups of performance curves recorded 986 at $N_{r}=1,4$ and 7, respectively, for both the NEC and for the 987 end-to-end throughput, where $N_{r}$ is the maximum number of 988 MAC retransmissions. The performance figures recorded for 989 the infinite number of MAC retransmissions scenario, namely 990 for $N_{r}=\infty$ are identical for the theory evaluated from (5) 991 and for the simulations. All the analytical and the simulation 992 based values recorded for the NEC increase, when the FER 993 increases. By contrast, the curves representing the end-to-end 994 throughput decrease, when the FER increases. The reason for 995 this observation is that a high FER in a link indicates a high 996 breakage probability not only for the specific link and but 997 also for the entire route, when retransmissions are required. 998 However, if $N_{r}$ is sufficiently high, then the success probability 999 of a packet across a link or even the entire route becomes higher.

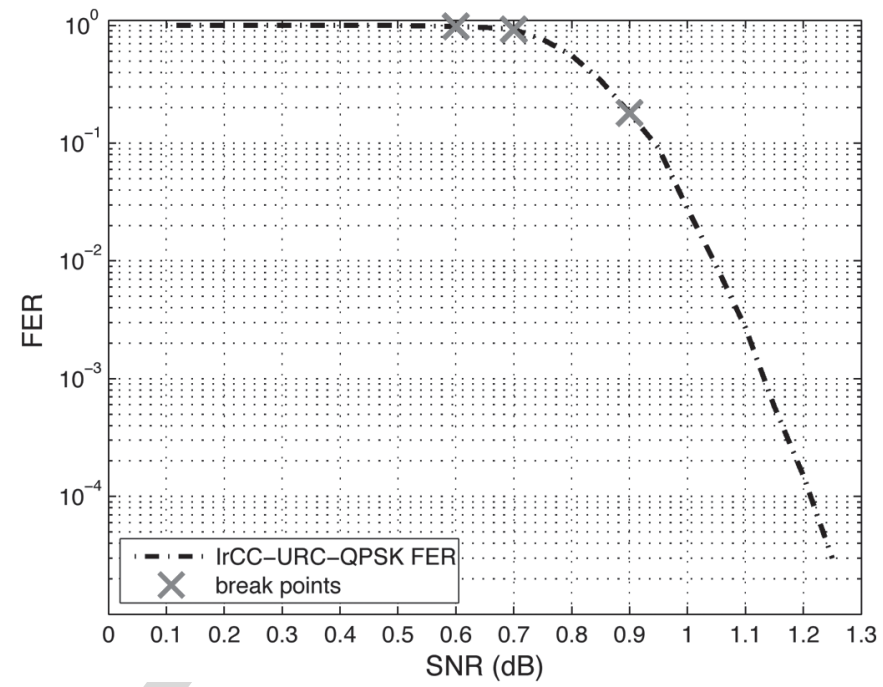

Fig. 22. FER versus SNR for the IrCC-URC-QPSK scheme of Section II-A for the average code rate of $R_{c}=0.5$ in an AWGN channel.

This trend is presented in Fig. 20, where the curve recorded for 1000 $N_{r}=7$ is seen to be close to that of $N_{r}=\infty$. The discrepancy 1001 between the theoretical value and the simulation-based value 1002 becomes higher when $N_{r}$ is reduced and simultaneously the 1003 FER is increased. Fig. 20 also shows that the theoretical energy 1004 consumption of (19) based on the energy-conscious OF is closer 1005 to the simulation based values than those based on the OF rely- 1006 ing on an infinite number of MAC retransmissions. Naturally, 1007 the advantage of the OF is more substantial for $N_{r}=1 . \quad 1008$

Fig. 21 also displays two groups of performance curves, 1009 one group for the NEC and the other group for the end-to- 1010 end throughput, which are associated with $H=2$ and 10, 1011 respectively. When $H$ is increased, the normalized energy 1012 consumption is reduced and the end-to-end throughput is de- 1013 creased, because the distance between a pair of adjacent nodes 1014 is reduced and therefore the transmit power required at each 1015 node for successfully delivering a packet is reduced. Similarly, 1016 as discussed in [73], the theoretical values estimated based 1017 on the proposed OF are closer to the simulated values than 1018 to those estimated on the basis of an infinite number of MAC 1019 retransmissions, especially when both $H$ and the FER are high. 1020

Hence, as elaborated on in [73], the conclusion is reached 1021 from Figs. 20 and 21 that the proposed energy-conscious OF 1022 is more accurate than the one assuming an infinite number of 1023 MAC retransmissions at high FERs, or for a high number of 1024 hops at a low maximum number of MAC retransmissions. 1025

\section{B. Traditional Routing With Adjustable Transmit Power}

1026

The FER curve was generated for the AWGN channel model 1027 with the aid of simulation [74]. According to the approach 1028 of [102], this will allow us to determine the average FER 1029 for arbitrary fading channels upon weighting the AWGN-FER 1030 by the Probability Distribution Function (PDF) of the fading 1031 channel and averaging it over the legitimate dynamic range. 1032 More specifically, the channel model considered is the uncor- 1033 related, non-dispersive Rayleigh fading channel. The average 1034 FER expression FER Rayleigh is determined for the Rayleigh 1035 fading channel considered by integrating the specific $F E R_{A W G N} 1036$ 
1037 value of the AWGN channel experienced at a given SNR after 1038 weighting it by the probability of that specific SNR, which is 1039 given by:

$$
F E R_{\text {Rayleigh }}=\int_{0}^{\infty} e^{-\gamma} F E R_{A W G N}(\gamma) d \gamma
$$

1040 where $\gamma$ is the channel SNR, $e^{-\gamma}$ represents the Rayleigh 1041 channel while the $F E R_{A W G N}(\gamma)$ versus the SNR curve is ap1042 proximated by the following four-segment FER vs. SNR model 1043 representing the AWGN channel:

$$
F E R_{A W G N}(\gamma) \approx \begin{cases}1, & \text { if } 0 \leq \gamma<\eta_{1}, \\ 10 a_{1} \log (\gamma)+a_{2}, & \text { if } \eta_{1} \leq \gamma<\eta_{2}, \\ 10 a_{3} \log (\gamma)+a_{4}, & \text { if } \eta_{2} \leq \gamma<\eta_{3}, \\ a_{5} e^{-10 a_{6} \log (\gamma)}, & \text { if } \gamma \geq \eta_{3},\end{cases}
$$

1044 with $\eta_{1}, \eta_{2}$ and $\eta_{3}$ being the break-points of the four-segment 1045 FER versus SNR approximation $F E R_{A W G N}(\gamma)$. Eqs. (21) and 1046 (22) are suitable for approximating different FER curves by ap1047 propriately setting the corresponding parameter values invoked. 1048 Eq. (21) may be readily extended to arbitrary channel models. 1049 Given $F E R_{\text {Rayleigh }}$, the successful reception probability of a 1050 link can be calculated as $\left[1-\left(F E R_{\text {Rayleigh }}\right)^{N_{r}}\right]$ if the maximum 1051 number of MAC retransmissions (including the first MAC 1052 retransmission attempt) is $N_{r}$. Specifically, for the IrCC-URC1053 QPSK scheme of Section II-A[72] employed, $a_{1}=-0.5889$, $1054 a_{2}=1.3341, a_{3}=-3.705, a_{4}=3.5169, a_{5}=4.4669 \times 10^{6}$ 1055 and $a_{6}=18.9118$. Additionally, the values of the break-points $1056 \eta_{1}, \eta_{2}$ and $\eta_{3}$ are determined for the SNR points of $0.6 \mathrm{~dB}$, $10570.7 \mathrm{~dB}$, and $0.9 \mathrm{~dB}$, which are based on the curves seen 1058 in Fig. 22. Fig. 22 shows the FER performance versus the 1059 SNR, when the IrCC-URC-QPSK scheme of Section II-A is 1060 employed, relying on the average code rate of $R_{c}=0.5$ in an 1061 AWGN channel. As seen from Fig. 22, the corresponding hori1062 zontal points of the symbol ' $x$ ' are $0.6 \mathrm{~dB}, 0.7 \mathrm{~dB}$ and $0.9 \mathrm{~dB}$. 1063 Therefore, by employing a practical coding scheme, such as 1064 the IrCC-URC-QPSK scheme of Section II with the aid of (19), 1065 it arrives at

$$
\bar{E}_{\text {total }}=\frac{P_{t_{1}}}{p_{1}} T
$$

1066 which shows that $\bar{E}_{\text {total }}$ is independent of the number of retrans1067 missions in a single-hop route. In this context, the NEC is the 1068 same as that of a transmitter operating without a transmission 1069 limit, i.e. when $N_{r}=$ inf. As indicated in [74], optimizing 1070 the transmit power of the source was formulated as a convex 1071 optimization problem.

1072 Once the closed-form expression of (23) for the NEC $\bar{E}_{\text {total }}$ 1073 of a single hop, the optimized transmit power $P_{t_{1}}$ may be 1074 calculated by setting the derivative of (23) with respect to $P_{t_{1}}$ 1075 to zero, which yields

$$
\begin{aligned}
\frac{1}{p_{1}}+\frac{P_{t_{1}}}{p_{1}^{2}} \frac{d\left(1-p_{1}\right)}{d P_{t_{1}}} & =0 \\
\frac{p_{1}}{-P_{t_{1}}} & =\frac{d\left(1-p_{1}\right)}{d P_{t_{1}}},
\end{aligned}
$$

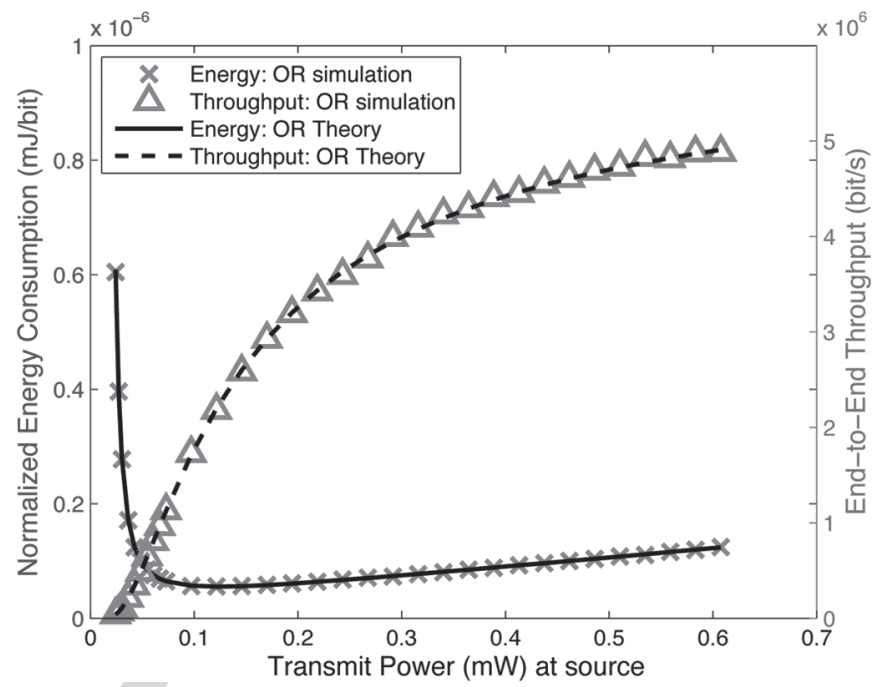

Fig. 23. The NEC $\bar{E}_{\text {total }}$ and the end-to-end throughput $R_{e 2 e}$ versus the transmit power $P_{t_{S}}$.

where $1-p_{1}=F E R_{1}$. Finally, the analytical expression of the 1076 optimized transmit power $P_{t_{1}}$ can be found. The existence of the 1077 optimized transmit power at the source of a single-hop route 1078 is shown in Fig. 23. Moreover, the end-to-end throughput $R_{e 2 e} 1079$ of the TR relying on an adjustable transmit power also obeys 1080 the same expression of (20). Therefore, the NEC $\bar{E}_{\text {total }}$ and the 1081 end-to-end throughput $R_{e 2 e}$ are compared both in terms of sim- 1082 ulation and theoretical results in Fig. 23, where the maximum 1083 number of MAC retransmissions is $N_{r}=7$. The frame length 1084 of the data packets, which are generated from the application 1085 layer, is 1024 Bytes. The $802.11 \mathrm{~g}$ standard is employed in the 1086 DL layer. The distance between $S$ and $D$ is $1000 \mathrm{~m}$. The other 1087 simulation configurations are listed in Table IV.

Fig. 23 shows that the NEC initially decreases and then 1089 increases slowly beyond the transmit power of $0.12 \mathrm{~mW}$, where 1090 $0.12 \mathrm{~mW}$ is the optimal transmit power obtained by using 1091 (24). The end-to-end throughput increases upon increasing the 1092 transmit power at $S$. Observe that the simulation results closely 1093 match the theoretical curve.

1094

The idealized multi-hop linear network researched in 1095 Section III-A may be extended to a more realistic random net- 1096 work relying on Dijkstra's routing algorithm [124] and invoking 1097 the NEC $\bar{E}_{\text {total }}$ for route selection. Hence, a heuristic routing 1098 algorithm, namely the TR having an adjustable transmit 1099 power is invoked in [74] (referred to as Algorithm 1 in [74]), 1100 which may be adapted to the random network scenario 1101 considered for guaranteeing a high energy efficiency. For 1102 ease of interpretation, in this paper, the TR having an 1103 adjustable transmit power is exemplified with the aid of 1104 its step-by-step execution using the NEC metric $\bar{E}_{\text {total }}$, as 1105 shown in Fig. 24. It is assumed that $\mathcal{V}$ is the vertex set, $v$ is 1106 a node in the set $\mathcal{V}$ and $\bar{E}_{S v}$ denotes the NEC. Moreover, $S 1107$ represents the set of selected nodes, while $P_{t}^{o p t}(u, v)$ denotes 1108 the optimal transmit power of node $u$ assigned for transmission 1109 to node $v$.

As an example, the positions of $S, D, R_{1}$ and $R_{2}$ are as- 1111 sumed to be $(100,100),(900,100),(500,500)$, and $(300,1112$ 400), respectively. The IrCC-URC-QPSK is employed in the 1113 


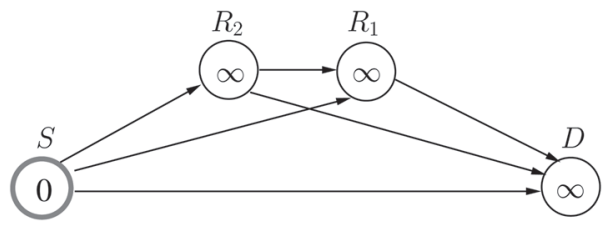

(a)

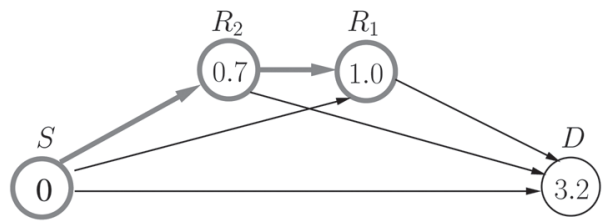

(c)

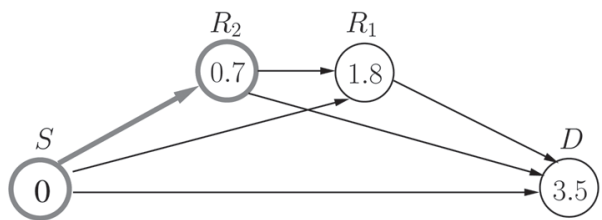

(b)

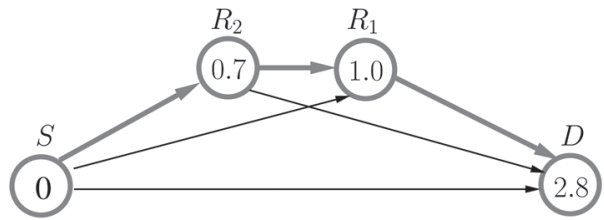

(d)

Fig. 24. Execution of the TR associated with an adjustable transmit power in a specific instance, where the positions of $S, D, R_{1}$ and $R_{2}$ are assumed to be $(100,100),(900,100),(500,500)$, and $(300,400)$, respectively. The value within a node $v$ is its energy cost $\bar{E}_{\text {total }}\left(\times 10^{-8} \mathrm{~mJ} / \mathrm{bit}\right)$ for transmission from $S$ to node $v$. After each iteration one node is incorporated into the set $S$. The nodes in boldface denote the nodes in $S$ after each iteration and the arrows in boldface represent the shortest route from $S$ to the nodes in boldface after each iteration. Due to the adjustable transmit power of node $u$, the probability $p_{f}(u, v)$ of a packet, which is dropped at any link $u-v$ after $N_{r}=7$ retransmissions, has nearly the same value of $p_{f}(u, v)=0.041$, hence this value next to the arrows is not plotted for simplicity. (a) The situation just after the initialization, where $S=\{S\}$. (b) The first iteration of the algorithm, where $S=\{S\}$ before the iteration, while after the iteration $R_{2}$ is incorporated into the set $S$ with the optimum power of $P_{t}^{o p t}\left(S, R_{2}\right)=0.16 \mathrm{~mW}$, yielding $S=\left\{S, R_{2}\right\}$. (c) The second iteration of the algorithm, where $S=\left\{S, R_{2}\right\}$ before the iteration, while after the iteration $R_{1}$ is incorporated into the set $S$ with the optimum power of $P_{t}^{o p t}\left(R_{2}, R_{1}\right)=0.06 \mathrm{~mW}$, yielding $\mathcal{S}=\left\{S, R_{2}, R_{1}\right\}$. (d) The final iteration of the algorithm, where $\mathcal{S}=\left\{S, R_{2}, R_{1}\right\}$ before the iteration, while after the iteration $D$ is incorporated into the set $S$ with the optimum power of $P_{t}^{o p t}\left(R_{1}, D\right)=0.39 \mathrm{~mW}$, yielding $S=\left\{S, R_{2}, R_{1}, D\right\}$. The algorithm terminates.

1114 PHY layer. The channel imposes both free-space path-loss and 1115 uncorrelated Rayleigh fading, plus the ubiquitous AWGN. The 1116 other relevant parameters are listed in Table IV. Each node is 1117 assumed to be aware of the other nodes' position, hence also 1118 of their distance. In a compact form, $\mathcal{V}=\left\{S, R_{1}, R_{2}, D\right\}$ and $1119 S=\{S\}$, as shown in Fig. 24(a). In Fig. 24(b), $S$ calculates its 1120 transmit power optimized for minimizing the NEC from (24), 1121 which is $\bar{E}_{S R_{1}}=1.8 \times 10^{-8} \mathrm{~mJ} / \mathrm{bit}, \bar{E}_{S R_{2}}=0.7 \times 10^{-8} \mathrm{~mJ} / \mathrm{bit}$, $1122 \bar{E}_{S D}=3.5 \times 10^{-8} \mathrm{~mJ} / \mathrm{bit}$ for transmission from $S$ to $R_{1}, R_{2}$ and $1123 \mathrm{D}$, respectively. Since $\bar{E}_{S R_{2}}=0.7 \times 10^{-8} \mathrm{~mJ} / \mathrm{bi}$ is the lowest 1124 in the set of the three energies, $S$ is updated to $\left\{S, R_{2}\right\}$. Then 1125 in Fig. 24(c), $R_{2}$ calculates its transmit power optimized for 1126 minimizing the NEC from (24) for the transmission, which 1127 is spanning from $S$ to node $R_{1}$ and $D$ via $R_{2}$, respectively. 1128 Since the updated NEC $\bar{E}_{S R_{1}}=1.0 \times 10^{-8} \mathrm{~mJ} / \mathrm{bit}$ is lower than $1129 \bar{E}_{S D}=3.2 \times 10^{-8} \mathrm{~mJ} / \mathrm{bit}, S$ is updated to $\left\{S, R_{2}, R_{1}\right\}$. Finally, in 1130 Fig. 24(d), $R_{1}$ adjusts its own transmit power to the optimal one, 1131 which minimizes the NEC $\bar{E}_{S D}=2.8 \times 10^{-8} \mathrm{~mJ} / \mathrm{bit}$ from $S$ to $1132 D$ via $R_{2}$ and $R_{1}$. At this stage, $D$ is incorporated into $S$. Since $1133 \mathcal{S}=\left\{S, R_{2}, R_{1}, D\right\}$, the TR with adjustable transmit power may 1134 be deemed to have converged and the route $S-R_{2}-R_{1}-D$ is 1135 deemed to be the optimal route for transmission from $S$ to $D$.

1136 The computational complexity has three main contributing 1137 factors: a) the calculation of a single NEC in a specific case; $1138 \mathrm{~b}$ ) the number of NEC calculations; c) and finally, finding the 1139 minimum NEC in each round. They denote the complexity of $1140 E_{s}, E_{f}$ and $p_{s}$, where $p_{s}=\prod_{1}^{H} B\left(p_{i}\right)$, by $C\left(E_{s}\right), C\left(E_{f}\right)$ and $1141 C\left(p_{s}\right)$. The complexity of evaluating $D_{s}$ and $D_{f}$ is the same 1142 as that of $E_{s}$ and $E_{f}$, apart from a multiplicative constant. 1143 The number of NEC calculations is given by the number of 1144 node pairs, which is $\mathcal{V}(\mathcal{V}-1) / 2$. The minimum NEC can be 1145 found based on the Fibonacci heap approach of [125], which 1146 has a complexity on the order of $\mathrm{O}(\log \mathcal{V})$. Therefore, the 1147 complexity imposed by the TR with adjustable transmit power 1148 is $\mathrm{O}\left[\mathcal{V}^{2}\left[\mathcal{C}\left(E_{s}\right)+C\left(E_{f}\right)+C\left(p_{s}\right)\right]+\mathcal{V} \log \mathcal{V}\right]$.
The performance of TR relying on an adjustable transmit 1149 power will be characterized in Section III-C in comparison to 1150 that of the OR of Section III-C.

\section{Opportunistic Routing With Adjustable Transmit Power}

The TR transmits the packet along the specific pre-selected 1153 route having the lowest estimated NEC. This pre-selected route 1154 is determined after the estimation and comparison of the NEC 1155 of each potential candidate route. The information invoked 1156 for routing decisions is gleaned during the process of route 1157 discovery, but this information may become stale owing to 1158 node-mobility. Instead, OR considers the potential chances of 1159 success for each candidate $\mathrm{RN}$, bearing in mind their time- 1160 variant channel conditions. Regardless of which particular RN 1161 receives the packet from the source successfully, if this RN has 1162 the highest priority in the forwarder RN list, it will forward the 1163 packet to the next RN. Naturally, the challenge in the design 1164 of the OR procedure is the beneficial selection of the forwarder 1165 RN set, the specific priority order of the potential forwarders 1166 and the avoidance of duplicate transmissions [110]. All the 1167 nodes in a node's neighbor list are assumed to belong to this 1168 node's forwarder R-list. The metric used for determining the 1169 priority order is the normalized energy required by this par- 1170 ticular RN for reaching D. Acknowledgement (ACK) packets 1171 are employed for avoiding the duplicate transmissions. The 1172 particular RN in the forwarder R-set, which has the highest 1173 priority owing to requiring the lowest energy will send the ACK 1174 first. The other RNs, which overhear the ACK will withdraw 1175 from the competition [126], [127].

A two-hop network is shown in Fig. 25, which has a sin- 1177 gle source $S$, a single destination $D$ and $M$ RNs $R_{1}, R_{2}, \cdots, 1178$ $R_{M-1}, R_{M} . S$ and $D$ are capable of communicating with all 1179 the RNs, as well as with each other. By contrast, the $M$ RNs 1180 are unable to communicate with each other. The idealized 1181 


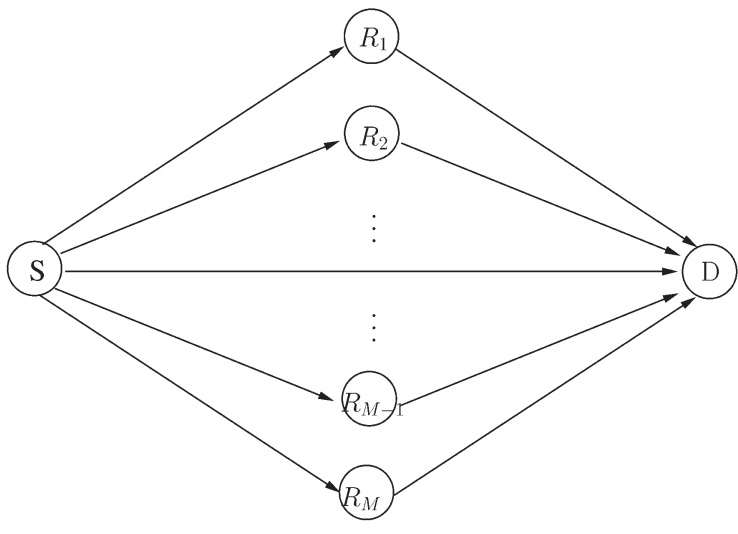

Fig. 25. A two-hop network assisted by a number of RNs.

1182 simplifying assumption is stipulated furthermore that each node 1183 knows the position of all other nodes. For each $\mathrm{RN} R_{m}, m=$ $11841 \ldots M$, the total average energy consumption $E_{R_{m} D}$ required 1185 for transmission from $R_{m}$ to $D$ is given by $E_{R_{m} D}=E_{R_{m} D}^{s}+E_{R_{m} D}^{f}$ 1186 where $E_{R_{m} D}^{s}$ represents the energy dissipated by a packet, which 1187 is successfully delivered from $R_{m}$ to $D$ while $E_{R_{m} D}^{f}$ represents 1188 the energy dissipated by a packet, which is dropped before 1189 reaching $D$ from $R_{m}$ after $N_{r}$ MAC retransmissions. Let $E_{S}$ 1190 denote the energy dissipated while sending a packet from 1191 the source $S$ to any of the RNs $R_{m}$, which is $E_{S}=P_{t_{S}} T$. It 1192 is assumed that $E_{R_{1} D}<E_{R_{2} D}<\cdots<E_{R_{M} D}$. Furthermore, for 1193 convenience, the destination node $D$ is represented as $R_{0}$ and $1194 \prod_{m=0}^{M}\left(1-p_{S R_{m}}\right)=\zeta$, where $p_{S R_{m}}$ denotes the probability of a 1195 packet, which is successfully delivered from $S$ to $R_{m}$.

1196 If $S$ successfully sends a packet to the $m$-th $\mathrm{RN}, m=$ $11970,1, \ldots, M$, with the aid of $n_{r}$ transmissions, the probability of 1198 this event is

$$
\begin{aligned}
& p_{0}\left(n_{r}\right)=\zeta^{n_{r}-1} p_{S R_{0}}, \text { if } m=0 \\
& p_{m}\left(n_{r}\right)=\zeta^{n_{r}-1} \prod_{i=0}^{m-1}\left(1-p_{S R_{i}}\right) p_{S R_{m}}, \text { if } 1 \leq m \leq M .
\end{aligned}
$$

1199 Correspondingly, the energy dissipated becomes

$$
\begin{aligned}
& E_{0}\left(n_{r}\right)=n_{r} E_{S}, \text { if } m=0 \\
& E_{m}\left(n_{r}\right)=n_{r} E_{S}+E_{R_{m} D}, \text { if } 1 \leq m \leq M
\end{aligned}
$$

1200 Let $D_{R_{m} D}$ denote the average delay of a packet travers1201 ing from $R_{m}, m=1, \ldots, M$, to $D$, including the delay $D_{R_{m} D}^{s}$ 1202 encountered by a packet that is successfully delivered to $D$ 1203 and the delay $D_{R_{m} D}^{f}$ experienced when a packet is dropped 1204 before reaching $D$. Then $D_{R_{m} D}=D_{R_{m} D}^{s}+D_{R_{m} D}^{f}$, where $D_{R_{m} D}^{s}$ 1205 represents $D_{s}$ and $D_{R_{m} D}^{f}$ corresponds to $D_{f}$, provided that the 1206 number of hops is 1 . Consequently,

$$
\begin{aligned}
D_{0}\left(n_{r}\right) & =n_{r} D_{S}, \text { if } m=0 \\
D_{m}\left(n_{r}\right) & =n_{r} D_{S}+D_{R_{m} D} \\
& =n_{r} D_{S}+\left(D_{R_{m} D}^{S}+D_{R_{m} D}^{f}\right) \text {, if } 1 \leq m \leq M,
\end{aligned}
$$

1207 where $D_{S}$ is $T$, which denotes the duration of a Time Slot (TS).
Consequently, when taking into account all the possible 1208 events, the total energy consumption is

$$
\begin{aligned}
E_{\text {total }}=\sum_{n_{r}=1}^{N_{r}} p_{0}\left(n_{r}\right) & E_{0}\left(n_{r}\right) \\
& +\sum_{n_{r}=1}^{N_{r}} \sum_{m=1}^{M} p_{m}\left(n_{r}\right) E_{m}\left(n_{r}\right)+\zeta^{N_{r}}\left(N_{r} E_{S}\right)
\end{aligned}
$$

while the total delay becomes:

$$
\begin{aligned}
D_{\text {total }}=\sum_{n_{r}=1}^{N_{r}} p_{0}\left(n_{r}\right) & D_{0}\left(n_{r}\right) \\
& +\sum_{n_{r}=1}^{N_{r}} \sum_{m=1}^{M} p_{m}\left(n_{r}\right) D_{m}\left(n_{r}\right)+\zeta^{N_{r}}\left(N_{r} D_{S}\right)
\end{aligned}
$$

Meanwhile, the packet transmitted from $S$ may be dropped 1211 in the $S-D, S-R_{m}$ or $R_{m}-D$ link, where $m=1, \ldots, M$ and 1212 again, the destination can be replaced by $R_{0}$. Then the end-to- 1213 end outage probability $p_{f}$ may be formulated as

$$
p_{f}=p_{f, S-R_{0}}+p_{f, S-R_{m}}+p_{f, R_{m}-D}, m=1, \ldots, M
$$

Furthermore, the NEC $\bar{E}_{\text {total }}$ may be formulated as

$$
\bar{E}_{\text {total }}=\frac{E_{\text {total }}}{1-p_{f}},
$$

while the end-to-end throughput $R_{e 2 e}$ is given by

$$
R_{e 2 e}=\frac{1-p_{f}}{D_{\text {total }}} .
$$

Although the network topology in Fig. 25 has only two hops, 1217 this algorithm may be extended to a large network, where 1218 the OR principle is employed for each hop. Meanwhile, the 1219 optimal transmit power of each node is found for the sake 1220 of minimizing the NEC required for the successful passage 1221 of a packet from that node to the destination. Therefore, the 1222 heuristic routing algorithm, namely the $\mathrm{OR}$ associated with 1223 an adjustable transmit power is conceived in [74] (referred 1224 to as Algorithm 2 in [74]), for calculating the minimum NEC 1225 by carrying out optimum distance-dependent power alloca- 1226 tion at each node, hop-by-hop. For ease of interpretation, in 1227 this paper, the OR having an adjustable transmit power is 1228 exemplified with the aid of its step-by-step execution using 1229 the NEC metric $\bar{E}_{\text {total }}$, as shown in Fig. 26. Here, for any 1230 node $v$ in a given vertex set $\mathcal{V}, \bar{E}_{v D}$ denotes the NEC $\bar{E}_{\text {total }} 1231$ necessitated for transmission from node $v$ to the destination 1232 $D$, where the potential set of receiver nodes is denoted by 1233 $\mathcal{R}$. Furthermore, $P_{t}^{o p t}(v)$ is the optimal transmit power, which 1234 minimizes the NEC required for transmission from node $v$ to 1235 the destination $D$.

Again, as a specific example, both the topology and the 1237 relevant parameters used in Fig. 26 are similar to those used 1238 in Fig. 24. It was also assumed that each node is aware of 1239 the other nodes' position, hence also of their distance. In a 1240 compact form, $\mathcal{V}=\left\{S, R_{1}, R_{2}, D\right\}$ and $\mathcal{R}=\{D\}$, as shown in 1241 


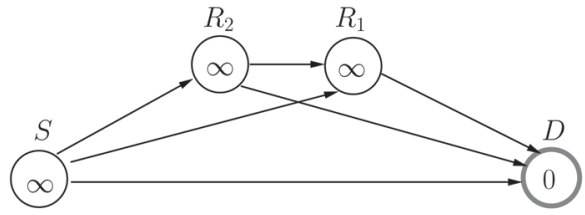

(a)

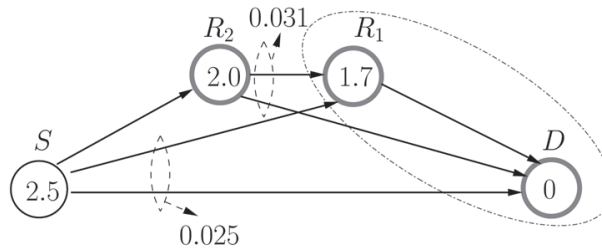

(c)

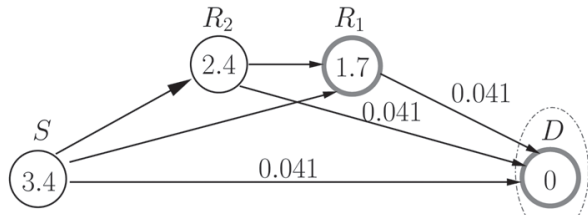

(b)

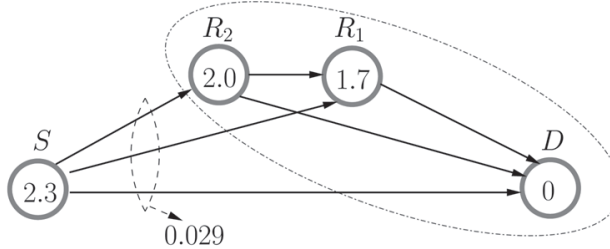

(d)

Fig. 26. Execution of the OR associated with an adjustable transmit power in a specific instance, where the positions of $S, D, R_{1}$ and $R_{2}$ are assumed to be $(100,100),(900,100),(500,500)$ and $(300,400)$, respectively. The value within a node $u$ is its cost $\bar{E}_{\text {total }}\left(\times 10^{-8} \mathrm{~mJ} / \mathrm{bit}\right)$ incurred by its transmission from node $u$ to $D$ and the dash-dot ellipse represents the receiver set $\mathcal{R}$ before each iteration. After each iteration one node is incorporated into the set $\mathcal{R}$. The nodes in boldface denote the nodes in $\mathcal{R}$ after each iteration. The values next to the arrows or the dashed ellipses represent the probability $p_{f}(u, \mathcal{R})$ of a packet being transmitted from $S$ in conjunction with the event that none of the nodes in the receiver set $\mathcal{R}$ receives it after $N_{r}=7$ retransmissions. (a) The situation just after the initialization, where $R=\{D\}$. (b) The first iteration of the algorithm, where $R=\{D\}$ before the iteration, while after the iteration $R_{1}$ is incorporated into the set $\mathcal{R}$ with the optimum power of $P_{t}^{\text {opt }}\left(R_{1}\right)=0.39 \mathrm{~mW}$, yielding $\mathcal{R}=\left\{R_{1}, D\right\}$. (c) The second iteration of the algorithm, where $\mathcal{R}=\left\{R_{1}, D\right\}$ before the iteration while after the iteration $R_{2}$ is incorporated into the set $\mathcal{R}$ with the optimum power of $P_{t}^{o p t}\left(R_{2}\right)=0.36 \mathrm{~mW}$, yielding $\mathcal{R}=\left\{R_{2}, R_{1}, D\right\}$. (d) The final iteration of algorithm, where $\mathcal{R}=\left\{R_{2}, R_{1}, D\right\}$ before the iteration, while after the iteration $S$ is incorporated into the set $\mathcal{R}$ with the optimum power of $P_{t}^{\text {opt }}(S)=0.41$ mW, yielding $\mathcal{R}=\left\{S, R_{2}, R_{1}, D\right\}$. The algorithm terminates.

1242 Fig. 26(a). In Fig. 26(b), $S, R_{1}$ and $R_{2}$ calculate their transmit 1243 power optimized for minimizing the NEC from (34), yielding $1244 \bar{E}_{S D}=3.4 \times 10^{-8} \mathrm{~mJ} / \mathrm{bit}, \bar{E}_{R_{1} D}=1.7 \times 10^{-8} \mathrm{~mJ} / \mathrm{bit}, \bar{E}_{R_{2} D}=$ $12452.4 \times 10^{-8} \mathrm{~mJ} /$ bit for transmission to $D$. Since $\bar{E}_{R_{1} D}=1.7 \times$ $124610^{-8} \mathrm{~mJ} / \mathrm{bit}$ is the lowest in the set of the three energies, $R$ is 1247 updated to $\left\{R_{1}, D\right\}$. Then in Fig. 26(c), $S$ and $R_{2}$ adjust their 1248 own transmit power and update their NEC for transmission 1249 to node $D$ by considering $\left\{R_{1}, D\right\}$ as their forwarder relay 1250 set. Since $\bar{E}_{R_{2} D}=2.0 \times 10^{-8} \mathrm{~mJ} / \mathrm{bit}$ is lower than $\bar{E}_{S D}=$ $12512.5 \times 10^{-8} \mathrm{~mJ} / \mathrm{bit}, \mathcal{R}$ is updated to $\left\{R_{2}, R_{1}, D\right\}$. Finally, in 1252 Fig. 26(d), $S$ adjusts its own transmit power to the optimal one, 1253 which minimizes $\bar{E}_{S D}=2.3 \times 10^{-8} \mathrm{~mJ} / \mathrm{bit}$, where $\left\{R_{2}, R_{1}, D\right\}$ 1254 is the resultant forwarder relay set. At this stage, the OR with 1255 adjustable transmit power may be deemed to have converged, 1256 since $S$ is incorporated into $\mathcal{R}$ and $\mathcal{R}=\left\{S, R_{2}, R_{1}, D\right\}$. In this 1257 algorithm, every node has to find its own forwarder $\mathrm{R}$-set 1258 by itself upon exploiting the knowledge of the other nodes' 1259 positions. If more than one node in a node's forwarder R1260 list receives the packet from that node successfully, then that 1261 particular one, which requires the lowest NEC for transmission 1262 to the destination has the highest priority for forwarding this 1263 packet. The nodes of the forwarder R-set communicate with 1264 each other similarly to the technique of [126] and again, the 1265 NEC required for successful transmission to $D$ is invoked for 1266 deciding the priority order of the forwarders.

1267 The complexity of finding the transmit power and the for1268 warder set also depends on three contributing factors, just like 1269 for the TR scenario. They denote the complexity of $E_{\text {total }}$ in 1270 (34) and of $p_{f}$ in (33) by $C\left(E_{\text {total }}\right)$ and $C\left(p_{f}\right)$, respectively. 1271 The OR with adjustable transmit power has to invoke $\mathcal{V}$ times 1272 for the sake of adding a further node into $R$ in each round. The 1273 optimal transmit power of any node in $(\mathcal{V}-\mathcal{R})$ is calculated 1274 in a specific round and the complexity of this calculation is 1275 given by $C\left(E_{\text {total }}\right)+C\left(p_{f}\right)$. Again, the complexity of finding 1276 the optimal transmit power can be calculated by Fibonacci heap [125] which has a complexity on the order of $\mathrm{O}(\log \mathcal{V}) .1277$ Therefore, the complexity of the OR with adjustable transmit 1278 power is $\mathrm{O}\left[\mathcal{V}^{2}\left[C\left(E_{\text {total }}\right)+C\left(p_{f}\right)\right]+V \log \mathcal{V}\right]$.

Now the performance of the networks associated with a total 1280 of $N=4$ and 15 nodes are analyzed. The positions of $S$ and 1281 $D$ are $(100,100)$ and $(900,100)$, respectively, while the other 1282 nodes are uniformly located within a circle centered at $(400,1283$ $100)$ with a radius of $400 \mathrm{~m}$. The NEC $\bar{E}_{\text {total }}$ and the end- 1284 to-end throughput $R_{e 2 e}$ are shown in Fig. 27 and Fig. 28 as 1285 a function of the maximum number of MAC retransmissions 1286 $N_{r}$. The theoretical NEC bound of both TR and OR was also 1287 invesgated when $N=4$, which was found by the exhaustive 1288 search of all the routes spanning from $S$ to $D$, while for $N=151289$ no theoretical bounds were given, since the exhaustive search 1290 has an excessive computation complexity.

1291

Fig. 27 shows that the performance of the energy- 1292 consumption OF based algorithm is close to the theoretical 1293 bound when $N=4$, especially in the case of a high $N_{r}$. Both 1294 Figs. 27 and 28 show that the energy-efficient OR outperforms 1295 both the Adjustable Energy-Efficient Opportunistic Routing 1296 (A-EEOR) algorithm defined in [91] and the energy-efficient 1297 TR. Here, the A-EEOR algorithm selects and prioritizes the 1298 forwarding set during the initialization stage according to the 1299 total energy cost of forwarding a packet to the destination node, 1300 which is estimated under the assumption of allowing a poten- 1301 tially infinite number of MAC retransmissions $N_{r}$. However, 1302 $N_{r}$ is finite in practical scenarios. Hence, more specifically, 1303 compared to the A-EEOR algorithm the OR algorithm has 1304 a lower normalized energy consumption for $N_{r}<4$, as seen 1305 in Fig. 27, while exhibiting a higher end-to-end throughput 1306 for $N_{r}<6$, as shown in Fig. 28. Moreover, both the OR and 1307 TR simulation results closely match the theoretical curves. 1308 When $N_{r}=1$ or 2 for the network topology of $N=4$, both 1309 the exhaustive search, labelled by "TR bound" and the TR 1310 algorithm proposed in [74], labelled by "TR theory", selected 1311 


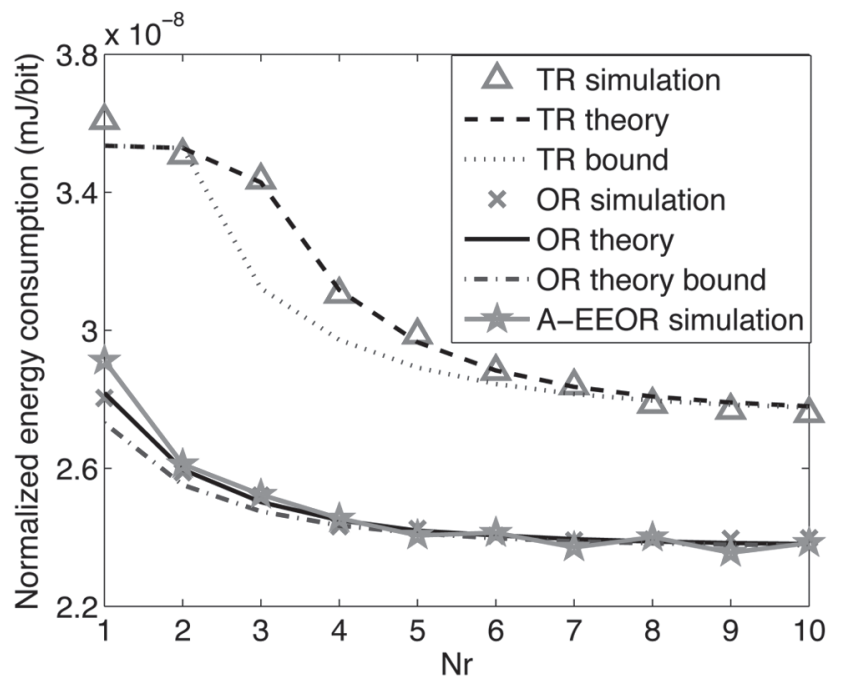

(a)

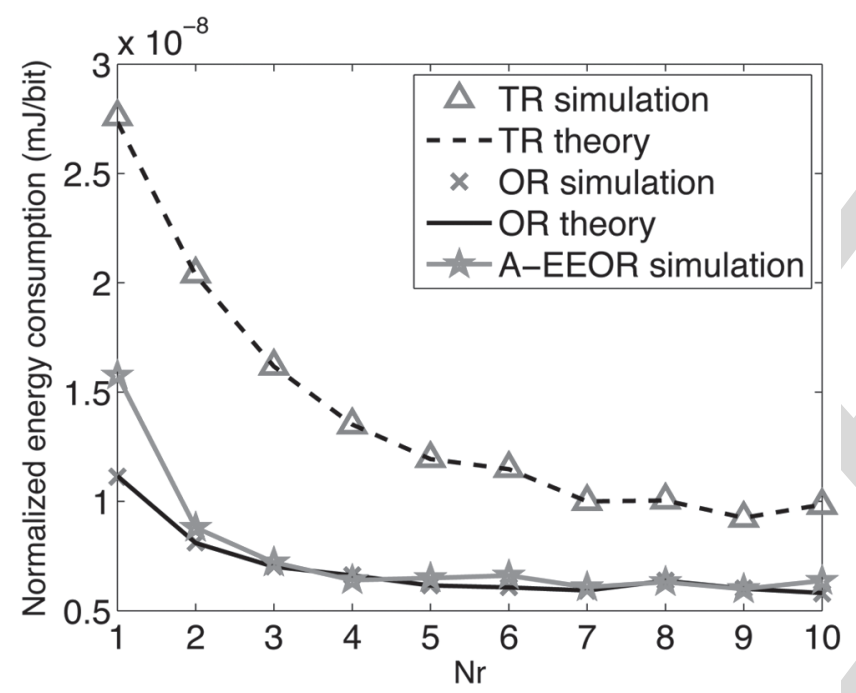

(b)

Fig. 27. The NEC $\bar{E}_{\text {total }}$ versus the maximum number of MAC retransmissions $N_{r}$ when $N=4$ and 15 . (a) Network topology $N=4$. (b) Network topology $N=15$.

1312 the route ' $\mathrm{S}-\mathrm{D}$ '. Hence the NEC is the same for both. When $13132<N_{r}<8$, the exhaustive search and the TR algorithm 1314 proposed in [74] choose different routes, since the exhaustive 1315 search represents the globally optimal algorithm, while the TR 1316 algorithm is a locally optimal algorithm. More specifically, the 1317 TR algorithm is optimal for every single hop. Moreover, the 1318 simulation results corresponding to the 'TR simulation' label 1319 closely match the theoretical value represented by the label 1320 'TR theory'. Therefore, the 'TR simulation/theory' and the 'TR 1321 bound' scenarios exhibit a performance discrepancy, when $2<$ $1322 N_{r}<8$, as seen in Fig. 27. Note that the NEC $\bar{E}_{\text {total }}$ decreases 1323 upon increasing $N_{r}$. However, the end-to-end throughput $R_{e 2 e}$ of 1324 the A-EEOR and OR regimes first increases and then saturates. 1325 Additionally, the end-to-end throughput of TR is in fact higher 1326 than that of OR for $N_{r}=1$ and 2 when $N=4$, but it is lower 1327 for $N_{r} \geq 3$, as seen in Fig. 28. This is because in case of a low 1328 number of MAC retransmissions, the direct near-line-of-sight 1329 route spanning from $S$ to $D$ in the TR has a more dominant 1330 priority than the other routes.

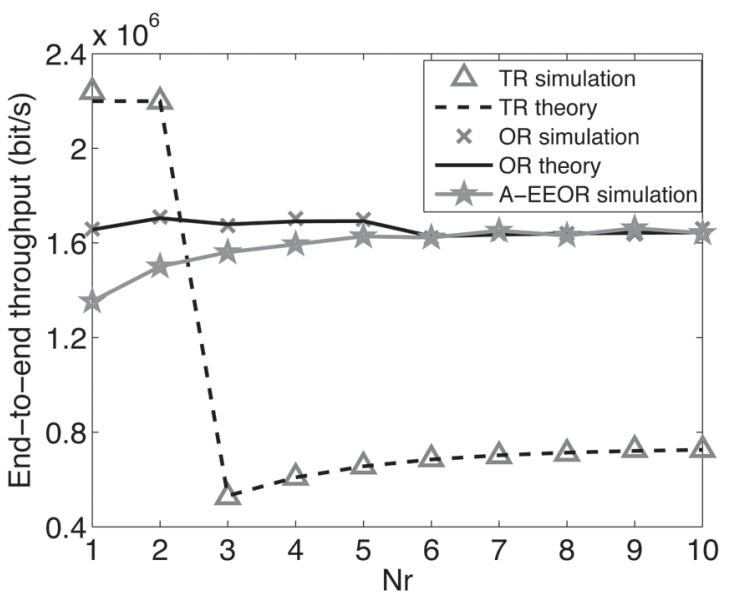

(a)

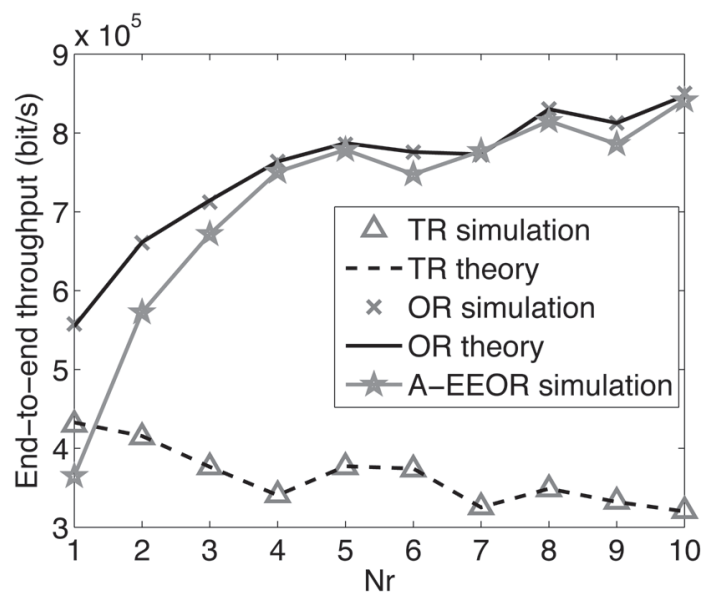

(b)

Fig. 28. The end-to-end throughput $R_{e 2 e}$ versus the maximum number of MAC retransmissions $N_{r}$ when $N=4$ and 15 . (a) Network topology $N=4$. (b) Network topology $N=15$.

\section{Conclusions And Design Guidelines}

\section{A. Conclusions}

In this paper diverse routing schemes were studied, investi- 1333 gating the benefits of multi-antenna aided RNs, the FER, the 1334 number of MAC retransmissions and the number of hops on 1335 the performance energy consumption.

- In Section I, we described the main functions of the OSI 1337 model layer by layer, then we highlighted the common 1338 methods of cross-layer design. The historical develop- 1339 ment of cross-layer aided routing protocol designs was 1340 portrayed in Table II. Then, we categorized the family 1341 of ad hoc routing protocols, which were improved in the 1342 following chapters.

- In Section II, we focused our attention on the reduction 1344 of the energy consumption by exploiting the benefits of 1345 the coordination between the PHY layer and the NET 1346 layer. Specifically, the advantages of near-capacity coding 1347 schemes were quantified in terms of their energy saving. A 1348 near-capacity three-stage concatenated IrCC-URC-STTC 1349 relay-transceiver equipped with two transmit antennas was 1350 proposed in [72] for the ad hoc network considered, 1351 since it achieved a low FER at a low transmit power. 1352 


\section{B. Design Guidelines}

1368 In general, three basic steps may be identified, when design1369 ing routing algorithms in ad hoc networks, which are:

The high effective transmission range of the IrCC-URCSTTC aided MA-RNs facilitated cross-layer design for activating beneficial routes having the lowest number of longer hops.

- Section III was specifically dedicated to minimizing the energy consumed by the data packets during the process of data transmission, where the NEC was quantified by considering both the PHY layer as well as the DL layer and the NET layer. Additionally, a cross-layer operation aided energy-efficient OR algorithm for ad hoc networks and an energy-consumption-based OF combined with PA was analyzed, which was proposed in [74] both for finding a theoretical bound and for conveying the packets through the network.

1) Determining the design targets, such as the network's throughput and/or energy consumption;

2) Determining the key factors, which influence the design targets most crucially. These key factors may be related to different layers, including the channel categories, the protocol parameters and so on;

3) Determining the routing metrics used for making routing decisions, such as the number of hops and/or the normalized end-to-end energy consumption.

Let us now detail these three design steps as follows:

- Throughput and energy consumption constitute a pair of important specifications in analyzing a network's performance, which critically depend on the parameters of the different OSI layers. Hence, combining the functions of multiple layers with the aid of cross-layer operation is beneficial in terms of improving the attainable performance, as demonstrated in this tutorial with the assistance of several cross-layer aided routing algorithms designed for $a d$ hoc networks.

- The number of hops is one of the most popular routing metrics in routing design, as we demonstrated in the context of the classic routing algorithm, namely the DYMO protocol.

- One of the most important factors we have to consider in the PHY layer is constituted by the specific characteristics of the time-variant wireless channel, which inflict bit/symbol errors and even packet loss events at the receiver node. Hence, strong and robust channel coding schemes have to be employed for mitigating the channelinduced degradations. The BER and FER are the two representative parameters, which are capable of characterizing the influence of both the time-variant wireless channel and of the FEC coding schemes, hence representing the overall performance of the PHY layer.

- For the sake of reducing the system's total transmit energy consumption, a near-capacity coding scheme, such as the IrCC-URC-STTC scheme of Section II-A is the most appropriate choice, since it requires a reduced transmit power at a given BER/FER value, which may also be 1408 viewed as reducing the BER/FER at a given transmit 1409 power. This is the reason, why the IrCC-URC-STTC aided 1410 MA transceivers operate close to the achievable capacity 1411 and this is, why they are capable of reducing the num- 1412 ber of hops spanning from the source to the destination. 1413 Requiring less hops implies that less nodes are involved, 1414 hence reducing the energy dissipation. An energy-efficient 1415 routing algorithm relying on the employment of IrCC- 1416 URC-STTC aided MA transceivers [72] was analyzed in 1417 Section II-B and Section II-C, showing that the system's 1418 total transmit energy consumption was reduced.

1419

- Furthermore, having considered the factors influencing the 1420 design of both the PHY layer and of the NET layer, we 1421 have to proceed by characterizing the influence of the DL 1422 layer in the cross-layer aided routing design. Our goal 1423 is that of achieving a throughput improvement and for 1424 energy reduction. One of the representative factors in the 1425 DL layer is constituted by the number of maximum MAC 1426 retransmissions. The larger the number of maximum MAC 1427 retransmissions, the more energy will be consumed and 1428 the higher the delay becomes. As a benefit, the success- 1429 ful packet reception probability is improved. Hence, we 1430 have to find the most appropriate number of maximum 1431 MAC retransmissions for the sake of striking an attractive 1432 compromise.

1433

- Additionally, we emphasize that the energy assigned to 1434 the data packets plays a dominant role in determining the 1435 system's total energy dissipation, which hence has to be 1436 optimized. For the sake of achieving an improved network 1437 throughput and a reduced energy consumption, the joint 1438 influence of the FER in the PHY layer, of the maximum 1439 number of retransmissions in the DL layer and of the 1440 number of hops in the NET layer has to be carefully con- 1441 sidered. Additionally, opting for the NEC as the routing 1442 metric instead of the number of hops is more beneficial in 1443 terms of reducing energy consumption. Hence, an accurate 1444 energy-consumption-based $\mathrm{OF}$ is required for combining 1445 all the three factors corresponding to the lower three 1446 layers of the seven-layer OSI architecture, as indicated 1447 in Section III-A of the tutorial. The routing algorithm 1448 proposed strikes an attractive tradeoff between the normal- 1449 ized energy consumption and the end-to-end throughput 1450 in the context of real-world scenarios, as exemplified in 1451 Section III-A.

- A hop-length-dependent PA is beneficial in terms of re- 1453 ducing the energy consumption. If the transmit power of 1454 each node is assumed to be the same, a certain amount of 1455 extra energy will be dissipated, since the distance between 1456 each pair of nodes is different, which would necessitate a 1457 different amount of transmit energy. An energy-efficient 1458 TR algorithm was designed with the aid of the hop-length- 1459 dependent power allocation of Section III-B, which also 1460 jointly considered the FER in the PHY layer, the maximum 1461 number of retransmissions in the DL layer and the number 1462 of hops in the NET layer. A reliable routing metric is 1463 constituted by the NEC quantified in terms of the energy- 1464 based OF exemplified in Section III-B. 
1477 All operational systems rely on a vital form of cross1478 layer operation, which makes wireless systems different 1479 from wireline based systems. Explicitly, both handovers and 1480 power-control rely on cross-layer cooperation in all wireless 1481 systems. This is why they are usually shown diagrammati1482 cally as a block bridging the lowest three layers. Going back 1483 as far as the old second-generation GSM system, the total 1484 control-channel bitrate was as low as $961 \mathrm{bits} / \mathrm{sec}$, which 1485 limited the efficiency of the power-control and handover 1486 operations, especially at high velocity and for small traffic 1487 cells, when handovers are frequent. For the 3G systems the 1488 control-channel rates were increased by an order of mag1489 nitude to about $10 \mathrm{kbits} / \mathrm{s}$, which facilitated more prompt 1490 handovers and power-control actions, when for example 1491 the mobile turned at a street-corner. The 4G LTE system 1492 also followed this trend, since an increased control-channel 1493 rate supports more sophisticated cross-layer cooperation. 1494 Although the main focus of this tutorial is on the energy dis1495 sipated by data packets during the process of data transmission, 1496 we note that cross-layer cooperation imposes an extra network 1497 overhead, since the control information also plays an impor1498 tant role in determining the system's total energy consumption, 1499 especially in mobile scenarios where the control information 1500 assists in maintaining seamless communications [128]. The 1501 extra control information is generated, when the informa1502 tion exchange takes place amongst layers or different nodes, 1503 including the control bits and the extra control packets. 1504 Additionally, a plethora of control packets are required for both 1505 route discovery and for route maintenance. For example, node1506 mobility might cause the following problems:

1507

1508

1509

1510

1511

1512

1513

1514

1515

1516 Hence, the energy-consumption-based OFs formulated in the 1517 stationary scenario may require further adjustments, if the en1518 ergy dissipated by the control packets is also considered. It may 1519 be promising to employ bio-inspired algorithms, such as the 1520 ant colony algorithm [132], for accommodating a dynamically 1521 changing topology, which requires future research.
REFERENCES

[1] H. Labiod, Ed., Wireless Ad Hoc and Sensor Networks. Hoboken, NJ, 1523 USA: Wiley, 2008.

[2] R. Ramanathan and J. Redi, "A brief overview of Ad Hoc networks: 1525 Challenges and directions," IEEE Commun. Mag., vol. 40, no. 5, pp. 20- 1526 22, May 2002.

[3] H. Zimmermann, "OSI reference model-the ISO model of architecture 1528 for open systems interconnection," IEEE Trans. Commun., vol. 28, no. 4, 1529 pp. 425-432, Apr. 1980.

[4] Reference Model of Open Systems Interconnection, ISO/TC97/SC16 Std. 1531 Doc. N227, 1979.

[5] A. Goldsmith, Wireless Communications. New York, NY, USA: 1533 Cambridge Univ. Press, 2005.

[6] W. Stallings, Wireless Communications \& Networks, 2nd ed. 1535 Englewood Cliffs, NJ, USA: Prentice-Hall, 2005.

[7] Information Technology-Telecommunications and Information Exchange 1537 Between Systems-Local and Metropolitan Area Networks-Specific 1538 Requirements, IEEE Std. 802.11, 2007.

[8] "Internet protocol," IETF Draft, 1981.

[9] S. Deering and R. Hinden, "Internet protocol, version 6 (IPv6) specifica- 1541 tion," IETF Draft, 1998

[10] "Transmission control protocol," IETF Draft, 1981

[11] “User datagram protocol," IETF Draft, 1980.

[12] Definitions of Terms Related to Quality of Service, ITU-T E.800, 2008. 1545

[13] V. Srivastava and M. Motani, "Cross-layer design: A survey and the road 1546 ahead," IEEE Commun. Mag., vol. 43, no. 12, pp. 112-119, Dec. 2005. 1547

[14] M. Conti, G. Maselli, G. Turi, and S. Giordano, "Cross-layering in 1548 mobile Ad Hoc network design," Computer, vol. 37, no. 2, pp. 48-51, 1549 Feb. 2004.

[15] R. Jurdak, Wireless Ad Hoc and Sensor Networks: A Cross-layer Design 1551 Perspective. New York, NY, USA: Springer-Verlag, 2010.

[16] F. Fu and M. V. D. Schaar, "A new systematic framework for autonomous 1553 cross-layer optimization," IEEE Trans. Veh. Technol., vol. 58, no. 4, 1554 pp. 1887-1903, May 2009.

[17] R. Winter, J. H. Schiller, N. Nikaein, and C. Bonnet, "Crosstalk: Cross- 1556 layer decision support based on global knowledge," IEEE Commun. 1557 Mag., vol. 44, no. 1, pp. 93-99, Jan. 2006.

[18] E. Setton, T. Yoo, X. Zhu, A. Goldsmith, and B. Girod, "Crosslayer de- 1559 sign of Ad Hoc networks for real-time video streaming," IEEE Wireless 1560 Commun., vol. 12, no. 4, pp. 59-65, Aug. 2005.

[19] Q. Liu, X. Wang, and G. B. Giannakis, "A cross-layer scheduling al- 1562 gorithm with QoS support in wireless networks," IEEE Trans. Veh. 1563 Technol., vol. 55, no. 3, pp. 839-847, May 2006.

[20] W. L. Huang and K. B. Letaief, "Cross-layer scheduling and power con- 1565 trol combined with adaptive modulation for wireless Ad Hoc networks," 1566 IEEE Trans. Commun., vol. 55, no. 4, pp. 728-739, Apr. 2007.

[21] Q. Zhang and Y.-Q. Zhang, "Cross-layer design for QoS support in 1568 multihop wireless networks," Proc. IEEE, vol. 96, no. 1, pp. 64-76, 1569 Jan. 2008.

[22] B. J. Oh and C. W. Chen, "A cross-layer approach to multichannel MAC 1571 protocol design for video streaming over wireless Ad Hoc networks," 1572 IEEE Trans. Multimedia, vol. 11, no. 6, pp. 1052-1061, Oct. 2009.

[23] S. Chu and X. Wang, "Opportunistic and cooperative spatial multiplex- 1574 ing in MIMO Ad Hoc networks," IEEE/ACM Trans. Netw., vol. 18, no. 5, 1575 pp. 1610-1623, Oct. 2010.

[24] A. Ghosh and W. Hamouda, "Cross-layer antenna selection and channel 1577 allocation for MIMO cognitive radios," IEEE Trans. Wireless Commun., 1578 vol. 10, no. 11, pp. 3666-3674, Nov. 2011.

[25] M. Mardani, S.-J. Kim, and G. B. Giannakis, "Cross-layer design 1580 of wireless multihop random access networks," IEEE Trans. Signal 1581 Process., vol. 60, no. 5, pp. 2562-2574, May 2012.

[26] M. Uddin, C. Rosenberg, W. Zhuang, P. Mitran, and A. Girard, "Joint 1583 routing and medium access control in fixed random access wireless 1584 multihop networks," IEEE/ACM Trans. Netw., vol. 22, no. 1, pp. 80-93, 1585 Feb. 2014

[27] F. Tang, L. Barolli, and J. Li, "A joint design for distributed stable 1587 routing and channel assignment over multihop and multiflow mobile 1588 Ad Hoc cognitive networks," IEEE Trans. Ind. Informat., vol. 10, no. 2, 1589 pp. 1606-1615, May 2014.

[28] V. Kawadia and P. R. Kumar, "A cautionary perspective on cross-layer 1591 design," IEEE Wireless Commun., vol. 12, no. 1, pp. 3-11, Feb. 2005.

[29] E. M. Royer and C.-K. Toh, "A review of current routing protocols for 1593 Ad Hoc mobile wireless networks," IEEE Pers. Commun., vol. 6, no. 2, 1594 pp. 46-55, Apr. 1999.

[30] C. E. Perkins and P. Bhagwat, "Highly dynamic destination-sequenced 1596 distance vector (DSDV) for mobile computers," in Proc. ACM SIGCOMM, 1597 Aug. 31-Sep. 2, 1994, pp. 234-244. 
31] T. Clausen and P. Jacquet, Optimized Link State Routing Protocol (OLSR) (RFC 3626) 2003, IETF Draft.

[32] The Dynamic Source Routing Protocol (DSR) for Mobile Ad Hoc Networks for IPv4. [Online]. Available: http://tools.ietf.org/html/rfc 4728

[33] Ad hoc On-Demand Distance Vector (AODV) Routing. [Online]. Available: http://tools.ietf.org/html/rfc3561

[34] Dynamic MANET On-Demand (DYMO) Routing Routing. [Online]. Available: http://tools.ietf.org/html/draft-ietf-manet-dymo-19

[35] The Zone Routing Protocol (ZRP) for Ad Hoc Networks. [Online]. Available: http://tools.ietf.org/html/draft-ietf-manet-zone-zrp-04

[36] A. Goldsmith and S. B. Wicker, "Design challenges for energyconstrained Ad Hoc wireless networks," IEEE Wireless Commun., vol. 9, no. 4, pp. 8-27, Aug. 2002.

[37] M. R. Souryal, B. R. Vojcic, and R. L. Pickholtz, "Information efficiency of multihop packet radio networks with channel-adaptive routing," IEEE J. Sel. Areas Commun., vol. 23, no. 1, pp. 40-50, Jan. 2005.

[38] S.-H. Lee, E. Choi, and D.-H. Cho, "Timer-based broadcasting for power-aware routing in power-controlled wireless Ad Hoc networks," IEEE Commun. Lett., vol. 9, no. 3, pp. 222-224, Mar. 2005.

[39] M. Johansson and L. Xiao, "Cross-layer optimization of wireless networks using nonlinear column generation," IEEE Trans. Wireless Commun., vol. 5, no. 2, pp. 435-445, Feb. 2006.

[40] S. Mao et al., "On routing for multiple description video over wireless Ad Hoc networks," IEEE Trans. Multimedia, vol. 8, no. 5, pp. 10631074 , Oct. 2006

[41] A. Abdrabou and W. H. Zhuang, "A position-based QoS routing scheme for UWB mobile Ad Hoc networks," IEEE J. Sel. Areas Commun., vol. 24, no. 4, pp. 850-856, Apr. 2006.

[42] J. Zhang, Q. Zhang, B. Li, X. Luo, and W. Zhu, "Energy-efficient routing in mobile Ad Hoc networks: Mobility-assisted case," IEEE Trans. Veh. Technol., vol. 55, no. 1, pp. 369-379, Jan. 2006.

[43] S. Kompella, S. Mao, Y. T. Hou, and H. D. Sherali, "Cross-layer optimized multipath routing for video communications in wireless networks," IEEE J. Sel. Areas Commun., vol. 25, no. 4, pp. 831-840, May 2007.

[44] M. Chiang, S. H. Low, A. R. Calderbank, and J. C. Doyle, "Layering as optimization decomposition: A mathematical theory of network architectures," Proc. IEEE, vol. 95, no. 1, pp. 255-312, Jan. 2007.

[45] K. T. Phan, H. Jiang, C. Tellambura, S. A. Vorobyov, and R. Fan, "Joint medium access control, routing and energy distribution in multihop wireless networks," IEEE Trans. Wireless Commun., vol. 7, no. 12, pp. 5244-5249, Dec. 2008.

[46] J. Liu, Y. T. Hou, Y. Shi, and H. D. Sherali, "Cross-layer optimization for MIMO-based wireless Ad Hoc networks: Routing, power allocation, bandwidth allocation," IEEE J. Sel. Areas Commun., vol. 26, no. 6, pp. 913-926, Aug. 2008.

[47] A. Abdrabou and W. H. Zhuang, "Statistical QoS routing for IEEE 802.11 multihop Ad Hoc networks," IEEE Trans. Wireless Commun., vol. 8, no. 3, pp. 1542-1552, Mar. 2009.

[48] P. Li, Q. Shen, Y. Fang, and H. Zhang, "Power controlled network protocols for multi-rate Ad Hoc networks," IEEE Trans. Wireless Commun., vol. 8, no. 4, pp. 2142-2149, Apr. 2009.

[49] L. Ding, T. Melodia, S. N. Batalama, J. D. Matyjas, and M. J. Medley, "Cross-layer routing and dynamic spectrum allocation in cognitive radio Ad Hoc networks," IEEE Trans. Veh. Technol., vol. 59, no. 4, pp. 19691979, May 2010.

[50] Y. Lu, J. Guan, Z. Wei, and Q. Wu, "Joint channel assignment and crosslayer routing protocol for multi-radio multi-channel Ad Hoc networks," J. Syst. Eng. Electron., vol. 21, no. 6, pp. 1095-1102, Dec. 2010.

[51] Z. Ding and K. K. Leung, "Cross-layer routing using cooperative transmission in vehicular Ad-Hoc networks," IEEE Journal on Selected Areas in Communications, vol. 29, no. 3, pp. 571-581, Mar. 2011.

[52] B. Tavli and W. B. Heinzelman, "Energy-efficient real-time multicast routing in mobile Ad Hoc networks," IEEE Trans. Comput., vol. 60, no. 5, pp. 707-722, May 2011.

[53] S.-J. Syue, C.-L. Wang, T. Aguilar, V. Gauthier, and H. Afifi, "Cooperative geographic routing with radio coverage extension for SER constrained wireless relay networks," IEEE J. Sel. Areas Commun., vol. 30, no. 2, pp. 271-279, Feb. 2012.

[54] M. Pan, H. Yue, C. Zhang, and Y. Fang, "Path selection under budget constraints in multihop cognitive radio networks," IEEE Trans. Mobile Comput., vol. 12, no. 6, pp. 1133-1145, Jun. 2013.

[55] J. G. Li, D. Cordes, and J. Y. Zhang, "Power-aware routing protocols in Ad Hoc wireless networks," IEEE Wireless Commun., vol. 12, no. 6, pp. 69-81, Dec. 2005.

[56] S. D. Muruganathan, D. C. F. Ma, R. I. Bhasin, and A. Fapojuwo, "A centralized energy-efficient routing protocol for wireless sensor networks," IEEE Commun. Mag., vol. 43, no. 3, pp. S8-S13, Mar. 2005.
[57] J. Zhu, C. Qiao, and X. Wang, "On accurate energy consumption models 1677 for wireless Ad Hoc networks," IEEE Trans. Wireless Commun., vol. 5, 1678 no. 11, pp. 3077-3086, Nov. 2006.

1679 AQ2

[58] S. J. Baek and G. Veciana, "Spatial energy balancing through proactive 1680 multipath routing in wireless multihop networks," IEEE/ACM Trans. 1681 Netw., vol. 15, no. 1, pp. 93-104, Feb. 2007.

[59] S. Eidenbenz, G. Resta, and P. Santi, "The COMMIT protocol for 1683 truthful and cost-efficient routing in Ad Hoc networks with self- 1684 ish nodes," IEEE Trans. Mobile Comput., vol. 7, no. 1, pp. 19-33, 1685 Jan. 2008.

1686

[60] W. Liang, R. Brent, Y. Xu, and Q. Wang, "Minimum-energy all- 1687 toall multicasting in wireless Ad Hoc networks," IEEE Trans. Wireless 1688 Commun., vol. 8, no. 11, pp. 5490-5499, Nov. 2009.

1689

[61] M. Li, L. Ding, Y. Shao, Z. Zhang, and B. Li, "On reducing broadcast 1690 transmission cost and redundancy in Ad Hoc wireless networks using di- 1691 rectional antennas," IEEE Trans. Veh. Technol., vol. 59, no. 3, pp. 1433- 1692 1442, Mar. 2010.

1693

[62] C. Ma and Y. Yang, "A battery-aware scheme for routing in wireless 1694 Ad Hoc networks," IEEE Trans. Veh. Technol., vol. 60, no. 8, pp. 3919- 1695 3932, Oct. 2011.

[63] A. M. Akhtar, M. R. Nakhai, and A. H. Aghvami, "Power aware cooper- 1697 ative routing in wireless mesh networks," IEEE Commun. Lett., vol. 16, 1698 no. 5, pp. 670-673, May 2012.

1699

[64] T. Lu and J. Zhu, "Genetic algorithm for energy-efficient QoS multicast 1700 routing," IEEE Commun. Lett., vol. 17, no. 1, pp. 31-34, Jan. 2013.

[65] J. Vazifehdan, R. Prasad, and I. Niemegeers, "Energy-efficient reliable 1702 routing considering residual energy in wireless Ad Hoc networks," IEEE 1703 Trans. Mobile Comput., vol. 13, no. 2, pp. 434-447, Feb. 2014.

[66] G. Ferrari, S. A. Malvassori, and O. K. Tonguz, "On physical layeror- 1705 iented routing with power control in Ad Hoc wireless networks," IET 1706 Commun., vol. 2, no. 2, pp. 306-319, Feb. 2008.

1707

[67] J. C. Fricke, M. M. Butt, and P. A. Hoeher, "Quality-oriented adaptive 1708 forwarding for wireless relaying," IEEE Commun. Lett., vol. 12, no. 3, 1709 pp. 200-202, Mar. 2008.

[68] M. Haenggi and D. Puccinelli, "Routing in Ad Hoc networks: A case 1711 for long hops," IEEE Commun. Mag., vol. 43, no. 10, pp. 93-101, 1712 Oct. 2005.

[69] M. Sikora, J. N. Laneman, M. Haenggi, D. J. Costello, and T. E. Fuja, 1714 "Bandwidth-and power-efficient routing in linear wireless networks," 1715 IEEE Trans. Inf. Theory, vol. 52, no. 6, pp. 2624-2633, Jun. 2006.

[70] C. Bae and W. E. Stark, "End-to-end energy and bandwidth tradeoff in 1717 multihop wireless networks," IEEE Trans. Inf. Theory, vol. 55, no. 9, 1718 pp. 4051-4066, Sep. 2009.

1719

[71] J. Niu, L. Cheng, Y. Gu, L. Shu, and S. K. Das, "R3E: Reliable reactive 1720 routing enhancement for wireless sensor networks," IEEE Trans. Ind. 1721 Informat., vol. 10, no. 1, pp. 784-794, Feb. 2014.

[72] J. Zuo, H. V. Nguyen, S. X. Ng, and L. Hanzo, "Energy-efficient relay 1723 aided Ad Hoc networks using iteratively detected irregular convolu- 1724 tional coded, unity-rate coded and space-time trellis coded transceivers," 1725 in Proc. IEEE WCNC, Quintana-Roo, Mexico, Mar. 28-31, 2011, 1726 pp. $1179-1184$.

[73] J. Zuo, C. Dong, S. X. Ng, L.-L. Yang, and L. Hanzo, "Energy-efficient 1728 routing in Ad Hoc networks relying on channel state information and 1729 limited mac retransmissions," in Proc. IEEE VTC-Fall, San Francisco, 1730 CA, USA, Sep. 5-8, 2011, pp. 1-5.

[74] J. Zuo et al., "Cross-layer aided energy-efficient opportunistic routing in 1732 Ad Hoc networks," IEEE Trans. Commun., vol. 62, no. 2, pp. 522-535, 1733 Feb. 2014

[75] D. Feng et al., "A survey of energy-efficient wireless communications," 1735 IEEE Commun. Surveys Tuts., vol. 15, no. 1, pp. 167-178, 2013.

[76] M. C. Vuran and I. F. Akyildiz, "Error control in wireless sensor net- 1737 works: A cross layer analysis," IEEE/ACM Trans. Netw., vol. 17, no. 4, 1738 pp. 1186-1199, Aug. 2009.

[77] H. V. Nguyen, S. X. Ng, and L. Hanzo, "Distributed three-stage concate- 1740 nated irregular convolutional, unity-rate and space-time trellis coding for 1741 single-antenna aided cooperative communications," in Proc. IEEE 72nd 1742 VTC-Fall, Ottawa, ON, Canada, Sep. 6-9, 2010, pp. 1-5. 1743

[78] S. T. Brink, "Convergence behavior of iteratively decoded parallel con- 1744 catenated codes," IEEE Trans. Commun., vol. 49, no. 10, pp. 1727-1737, 1745 Oct. 2001.

[79] L. Hanzo, O. Alamri, M. El-Hajjar, and N. Wu, Near-Capacity Multi- 1747 Functional MIMO Systems. New York, NY, USA: Wiley, 2009. 1748

[80] User Datagram Protocol. [Online]. Available: http://tools:ietf:org/html/ 1749 rfc768

[81] L. Hanzo, S.-X. Ng, T. Keller, and W. Webb, Quadrature Amplitude 1751 Modulation: From Basics to Adaptive Trellis-Coded, Turbo-Equalised 1752 and Space-Time Coded OFDM, CDMA, and MC-CDMA Systems, 1753 2nd ed. Hoboken, NJ, USA: Wiley-IEEE Press, 2004. 
[82] J. Zuo, S. X. Ng, and L. Hanzo, "Fuzzy logic aided dynamic source routing in cross-layer operation assisted Ad Hoc networks," in Proc IEEE 72nd VTC-Fall, Ottawa, ON, Canada, Sep. 6-9, 2010, pp. 1-5.

83] A. Ibrahim, H. Zhu, and K. J. R. Liu, "Distributed energy-efficient cooperative routing in wireless networks," IEEE Trans. Wireless Commun., vol. 7, no. 10, pp. 3930-3941, Oct. 2008.

84] E. Baccarelli, M. Biagi, C. Pelizzoni, and N. Cordeschi, "Maximumrate node selection for power-limited multiantenna relay backbones," IEEE Trans. Mobile Comput., vol. 8, no. 6, pp. 807-820, Jun. 2009.

[85] C. Bae and W. E. Stark, "End-to-end energy-bandwidth tradeoff in multihop wireless networks," IEEE Trans. Inf. Theory, vol. 55, no. 9, pp. 4051-4066, Sep. 2009

[86] S. Banerjee and A. Misra, "Minimum energy paths for reliable communication in multi-hop wireless networks," in Proc. 3rd ACM Int. Symp. MobiHoc, Lausanne, Switzerland, Jun. 9-11, 2002, pp. 146-156.

87] S. Cui, R. Madan, A. Goldsmith, and S. Lall, "Cross-layer energy and delay optimization in small-scale sensor networks," IEEE Trans. Wireless Commun., vol. 6, no. 10, pp. 3688-3699, Oct. 2007.

[88] H. Alwan and A. Agarwal, "Multi-objective QoS routing for wireless sensor networks," in Proc. ICNC, Jan. 28-31, 2013, pp. 1074-1079.

[89] X.-Y. Li et al., "Reliable and energy-efficient routing for static wireless Ad Hoc networks with unreliable links," IEEE Trans. Parallel Distrib. Syst., vol. 20, no. 10, pp. 1408-1421, Oct. 2009.

[90] M. Zorzi and R. R. Rao, "Geographic random forwarding (GeRaF) for Ad Hoc and sensor networks: Energy and latency performance," IEEE Trans. Mobile Comput., vol. 2, no. 4, pp. 349-365, Oct.-Dec. 2003.

[91] X. Mao, S. Tang, X. Xu, X.-Y. Li, and H. Ma, "Energy-efficient opportunistic routing in wireless sensor networks," IEEE Trans. Parallel Distrib. Syst., vol. 22, no. 11, pp. 1934-1942, Nov. 2011.

92] M. Dehghan, M. Ghaderi, and D. Goeckel, "Minimum-energy cooperative routing in wireless networks with channel variations," IEEE Trans. Wireless Commun., vol. 10, no. 11, pp. 3813-3823, Nov. 2011.

[93] J. Zhu and X. Wang, "Model and protocol for energy-efficient routing over mobile Ad Hoc networks," IEEE Trans. Mobile Comput., vol. 10, no. 11, pp. 1546-1557, Nov. 2011.

[94] T. Luo, M. Motani, and V. Srinivasan, "Energy-efficient strategies for cooperative multichannel MAC protocols," IEEE Trans. Mobile Comput. vol. 11, no. 4, pp. 553-566, Apr. 2012.

95] S. Kwon and N. B. Shroff, "Energy-efficient SINR-based routing for multihop wireless networks," IEEE Trans. Mobile Comput., vol. 8, no. 5 pp. 668-681, May 2009.

96] C. Wei, C. Zhi, P. Fan, and K. B. Letaief, "AsOR: An energy efficient multi-hop opportunistic routing protocol for wireless sensor networks over Rayleigh fading channels," IEEE Trans. Wireless Commun., vol. 8, no. 5, pp. 2452-2463, May 2009.

[97] M. C. Vuran and I. F. Akyildiz, "XLP: A cross-layer protocol for efficient communication in wireless sensor networks," IEEE Trans. Mobile Comput., vol. 9, no. 11, pp. 1578-1591, Nov. 2010.

[98] H. Kwon, T. H. Kim, S. Choi, and B. G. Lee, "A cross-layer strategy for energy-efficient reliable delivery in wireless sensor networks," IEEE Trans. Wireless Commun., vol. 5, no. 12, pp. 3689-3699, Dec. 2006.

[99] A. N. Pantazis, S. A. Nikolidakis, and D. D. Vergados, "Energy efficient routing protocols in wireless sensor networks: A survey," IEEE Commun. Surveys Tuts., vol. 15, no. 2, pp. 551-591, 2013.

00] M. A. Rahman, S. Anwar, M. I. Pramanik, and M. F. Rahman, "A survey on energy efficient routing techniques in wireless sensor network," in Proc. 15th ICACT, Jan. 27-30, 2013, pp. 200-205.

01] S. Biswas and R. Morris, "Opportunistic routing in multi-hop wireless networks," ACM SIGCOMM Comput. Commun. Rev., vol. 34, no. 1, pp. 69-74, Jan. 2004.

02] Q. W. Liu, S. L. Zhou, and G. B. Giannakis, "Cross-layer combining of adaptive modulation and coding with truncated ARQ over wireless links," IEEE Trans. Wireless Commun., vol. 3, no. 5, pp. 1746-1755, Sep. 2004.

03] Z. Wang, Y. Chen, and C. Li, "CORMAN: A novel cooperative opportunistic routing scheme in mobile Ad Hoc networks," IEEE J. Sel. Areas Commun., vol. 30, no. 2, pp. 289-296, Feb. 2012.

104] A. M. Akhtar, M. R. Nakhai, and A. H. Aghvami, "On the use of cooperative physical layer network coding for energy efficient routing," IEEE Trans. Commun., vol. 61, no. 4, pp. 1498-1509, Apr. 2013.

05] R. C. Shah and J. M. Rabaey, "Energy aware routing for low energy Ad Hoc sensor networks," in Proc. IEEE Wireless Commun. Netw. Conf., Mar. 2002, vol. 1, pp. 350-355.

06] Y. Xu, J. Heidemann, and D. Estrin, "Geography-informed energy conservation for Ad Hoc routing," in Proc. 7th Annu. Int. Conf. MobiCom, Rome, Italy, Jul. 2001, pp. 70-84.

Q. F. Dong and S. Banerjee, "Minimum energy reliable paths using unreliable wireless links," in Proc. 6th ACM Int. Symp. MobiHoc, UrbanaChampaign, IL, USA, May 25-28, 2005, pp. 449-459.
[108] C.-E. Perkins, E.-M. Royer, S.-R. Das, and M.-K. Marina, "Performance 1833 comparison of two on-demand routing protocols for Ad Hoc networks," 1834 IEEE Pers. Commun., vol. 8, no. 1, pp. 16-28, Feb. 2001.

[109] M. Zorzi and R. R. Rao, "Geographic random forwarding (GeRaF) 1836 for Ad Hoc and sensor networks: Multihop performance," IEEE Trans. 1837 Mobile Comput., vol. 2, no. 4, pp. 337-348, Oct.-Dec. 2003.

[110] H. Liu, B. Zhang, H. Mouftah, X. Shen, and J. Ma, "Opportunistic 1839 routing for wireless Ad Hoc and sensor networks: Present and future 1840 24 directions," IEEE Commun. Mag., vol. 47, no. 12, pp. 103-109, 1841 Dec. 2009

[111] H. Dubois-Ferrière, M. Grossglauser, and M. Vetterli, "Valuable detours: 1843 Least-cost anypath routing," IEEE/ACM Trans. Netw., vol. 19, no. 2, 1844 pp. 333-346, Apr. 2011.

[112] A. A. Bhorkar, M. Naghshvar, T. Javidi, and B. D. Rao, "Adaptive 1846 opportunistic routing for wireless Ad Hoc networks," IEEE/ACM Trans. 1847 Netw., vol. 20, no. 1, pp. 243-256, Feb. 2012.

1848

[113] K. Zeng, Z. Yang, and W. Lou, "Location-aided opportunistic forwarding 1849 in multirate and multihop wireless networks," IEEE Trans. Veh. Technol., 1850 vol. 58, no. 6, pp. 3032-3040, Jul. 2009.

[114] R. Laufer, H. Dubois-Ferrière, and L. Kleinrock, "Polynomial- 1852 time algorithms for multirate anypath routing in wireless multihop 1853 networks," IEEE/ACM Trans. Netw., vol. 20, no. 3, pp. 742-755, 1854 Jun. 2012.

[115] L. Pelusi, A. Passarella, and M. Conti, “Opportunistic networking: Data 1856 forwarding in disconnected mobile Ad Hoc networks," IEEE Commun. 1857 Mag., vol. 44, no. 11, pp. 134-141, Nov. 2006.

[116] H. Khalife, N. Malouch, and S. Fdida, "Multihop cognitive radio net- 1859 works: To route or not to route," IEEE Netw., vol. 23, no. 4, pp. 20-25, 1860 Jul. 2009.

[117] K. C. Lee, U. Lee, and M. Gerla, "Geo-opportunistic routing for vehicu- 1862 lar networks [topics in automotive networking]," IEEE Commun. Mag., 1863 vol. 48, no. 5, pp. 164-170, May 2010.

[118] D. Wu, Y. Zhang, L. Bao, and A. C. Regan, "Location-based crowd- 1865 sourcing for vehicular communication in hybrid networks," IEEE Trans. 1866 Intell. Transp. Syst., vol. 14, no. 2, pp. 837-846, Jun. 2013.

[119] V. Conan, J. Leguay, and T. Friedman, "Fixed point opportunistic routing 1868 in delay tolerant networks," IEEE J. Sel. Areas Commun., vol. 26, no. 5, 1869 pp. 773-782, Jun. 2008.

[120] T. Spyropoulos, T. Turletti, and K. Obraczka, "Routing in delaytoler- 187 ant networks comprising heterogeneous node populations," IEEE Trans. 1872 Mobile Comput., vol. 8, no. 8, pp. 1132-1147, Aug. 2009.

[121] Y. Li et al., "Energy-efficient optimal opportunistic forwarding for delay- 1874 tolerant networks," IEEE Trans. Veh. Technol., vol. 59, no. 9, pp. 4500- 1875 4512, Nov. 2010.

[122] S.-G. Yoon, S. Jang, Y.-H. Kim, and S. Bahk, "Opportunistic routing 1877 for smart grid with power line communication access networks," IEEE 1878 Trans. Smart Grid, vol. 5, no. 1, pp. 303-311, Jan. 2014.

[123] T. H. Cormen, C. E. Leiserson, R. L. Rivest, and C. Stein, Introduc- 1880 tion to Algorithms, 3rd ed. Upper Saddle River, NJ, USA: MIT Press, 1881 2009.

[124] E. W. Dijkstra, "A note on two problems in connexion with graphs," 1883 Numerische Mathematik, vol. 1, no. 1, pp. 269-271, 1959.

[125] M. L. Fredman and R. E. Tarjan, "Fibonacci heaps and their uses in 1885 improved network optimization algorithms," J. Assoc. Comput. Mach., 1886 vol. 34, no. 3, pp. 596-615, Jul. 1987.

[126] C. Dong, L.-L. Yang, and L. Hanzo, "Multi-hop diversity aided multi- 1888 hop communications: A cumulative distribution function aware ap- 1889 proach," IEEE Trans. Commun., vol. 61, no. 11, pp. 4486-4499, 1890 Nov. 2013.

[127] C. Dong, L.-L. Yang, and L. Hanzo, "Performance analysis of multihop- 1892 diversity-aided multihop links," IEEE Trans. Veh. Technol., vol. 61, 1893 no. 6, pp. 2504-2516, Jul. 2012.

[128] W. C. Tan, S. K. Bose, and T.-H. Cheng, "Power and mobility aware 1895 routing in wireless Ad Hoc networks," IET Commun., vol. 6, no. 11, 1896 pp. 1425-1437, Jul. 2012.

1897

[129] F. D. Rango, F. Guerriero, and P. Fazio, "Link-stability and energy aware 1898 routing protocol in distributed wireless networks," IEEE Trans. Parallel 1899 Distrib. Syst., vol. 23, no. 4, pp. 713-726, Apr. 2012.

[130] A. A. Jeng and R.-H. Jan, "Adaptive topology control for mobile Ad Hoc 190 networks," IEEE Trans. Parallel Distrib. Syst., vol. 22, no. 12, pp. 1953- 1902 1960, Dec. 2011.

[131] G. Ferrari and O. K. Tonguz, "Impact of mobility on the BER perfor- 1904 mance of Ad Hoc wireless networks," IEEE Trans. Veh. Technol., vol. 56, 1905 no. 1, pp. 271-286, Jan. 2007.

1906

[132] S. L. Correia, J. Celestino, and O. Cherkaoui, "Mobility-aware 1907 ant colony optimization routing for vehicular Ad Hoc networks," in 1908 Proc. IEEE WCNC, Quintana-Roo, Mexico, Mar. 28-31, 2011, 1909 pp. 1125-1130. 


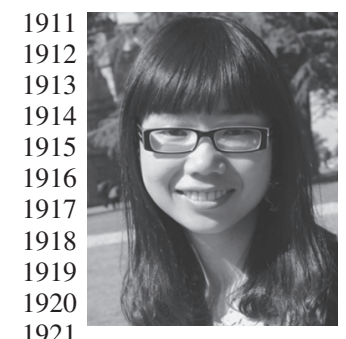

Jing Zuo received the B.Eng. degree in communications engineering and the M.Sc. degree in communications and information system from Jilin University, Changchun, China, in 2006 and 2008, respectively, and the Ph.D. degree in wireless communications from University of Southampton, U.K., in 2013. She is the recipient of scholarship under the UK-China Scholarships for Excellence programme from 2008 to 2011. From 2009 to 2013, she was involved in the OPTIMIX and CONCERTO European projects. She is currently with Huawei, Shenzhen, China and her 1922 current research interests include protocols and algorithms design, cross-layer 1923 optimization and opportunistic communications.

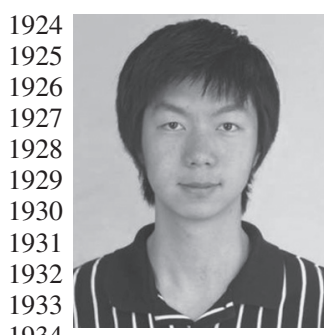

Chen Dong received the B.S. degree in electronic information sciences and technology from University of Science and Technology of China (USTC), Hefei, China, in 2004, and the M.Eng. degree in pattern recognition and automatic equipment from the University of Chinese Academy of Sciences, Beijing, China in 2007. He received the Ph.D. degree from the University of Southampton, UK, In 2014. Now he is a post-doc in the same University. He was the recipient of scholarship under the UK-China Scholarships for Excellence programme and he has been awarded 1935 Best Paper Award at IEEE VTC 2014-Fall. His research interests include 1936 applied math, relay system, channel modelling and cross-layer optimization.

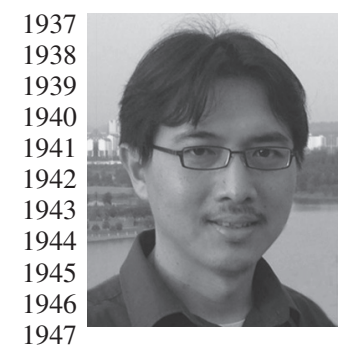

Soon Xin Ng (S'99-M'03-SM'08) received the B.Eng. degree (first class) in electronic engineering and the Ph.D. degree in telecommunications from the University of Southampton, Southampton, U.K., in 1999 and 2002, respectively. From 2003 to 2006, he was a postdoctoral research fellow working on collaborative European research projects known as SCOUT, NEWCOM and PHOENIX. Since August 2006, he has been a member of academic staff in the School of Electronics and Computer Science, University of Southampton. He is involved in the 1948 OPTIMIX and CONCERTO European projects as well as the IU-ATC and 1949 UC4G projects. He is currently an associate professor in telecommunications at 1950 the University of Southampton.

1951 His research interests include adaptive coded modulation, coded modula1952 tion, channel coding, space-time coding, joint source and channel coding, 1953 iterative detection, OFDM, MIMO, cooperative communications, distributed 1954 coding, quantum error correction codes and joint wireless-and-optical-fiber 1955 communications. He has published over 180 papers and co-authored two John 1956 Wiley/IEEE Press books in this field. He is a Chartered Engineer and a Fellow 1957 of the Higher Education Academy in the U.K.

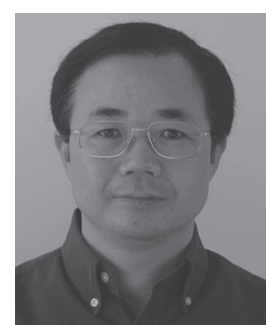

Lie-Liang Yang (M'98-SM'02) received the 1958 B.Eng. degree in communications engineering from 1959 Shanghai TieDao University, Shanghai, China, 1960 in 1988, and the M.Eng. and Ph.D. degrees in 1961 communications and electronics from Northern 1962 (Beijing) Jiaotong University, Beijing, China in 1963 1991 and 1997, respectively. From June 1997 to 1964 December 1997 he was a visiting scientist of the 1965 Institute of Radio Engineering and Electronics, 1966 Academy of Sciences of the Czech Republic. Since 1967 December 1997, he has been with the University 1968 of Southampton, United Kingdom, where he is the professor of wireless 1969 communications in the School of Electronics and Computer Science. His 1970 research has covered a wide range of topics in wireless communications, 1971 networking and signal processing. He has published over 300 research papers 1972 in journals and conference proceedings, authored/co-authored three books 1973 and also published several book chapters. The details about his publications 1974 can be found at http://www-mobile.ecs.soton.ac.uk/lly/. He is a Fellow of 1975 the IET, served as an associate editor to the IEEE TRANS. ON VEHICULAR 1976 TECHNOLOGY and Journal OF COMmuniCATIONS AND NeTwORKS 1977 (JCN), and is currently an associate editor to the IEEE Access and the Security 1978 and Communication Networks (SCN) Journal.

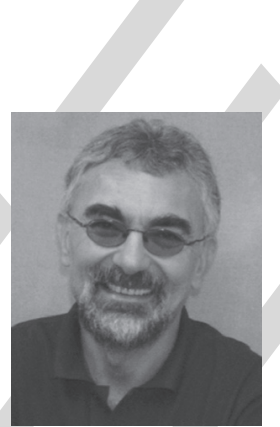

Lajos Hanzo received the degree in electronics in 1980 1976 and the doctorate degree in 1983. In 2009 he 1981 was awarded the honorary doctorate "Doctor Hon- 1982 oris Causa" by the Technical University of Budapest. 1983 During his 37-year career in telecommunications he 1984 has held various research and academic posts in 1985 Hungary, Germany and the UK. Since 1986 he has 1986 been with the School of Electronics and Computer 1987 Science, University of Southampton, UK, where he 1988 holds the chair in telecommunications. He has suc- 1989 cessfully supervised more than 80 Ph.D. students, 1990 co-authored 20 John Wiley/IEEE Press books on mobile radio communications 1991 totalling in excess of 10000 pages, published 1460 research entries at IEEE 1992 Xplore, acted both as TPC and General Chair of IEEE conferences, presented 1993 keynote lectures and has been awarded a number of distinctions. Currently 1994 he is directing a 60-strong academic research team, working on a range of 1995 research projects in the field of wireless multimedia communications sponsored 1996 by industry, the Engineering and Physical Sciences Research Council (EPSRC) 1997 UK, the European Research Council's Advanced Fellow Grant and the Royal 1998 Society's Wolfson Research Merit Award. He is an enthusiastic supporter of 1999 industrial and academic liaison and he offers a range of industrial courses. He 2000 is also a Governor of the IEEE VTS. During 2008-2012 he was the Editor-in- 2001 Chief of the IEEE Press and a Chaired Professor also at Tsinghua University, 2002 Beijing. His research is funded by the European Research Council's Senior 2003 Research Fellow Grant. For further information on research in progress and 2004 associated publications please refer to http://www-mobile.ecs.soton.ac.uk. 2005 


\section{AUTHOR QUERIES}

\section{AUTHOR PLEASE ANSWER ALL QUERIES}

AQ1 = Note that reference [26] and [105] are the same. Therefore, reference [105] was deleted from the list. Citations were renumbered accordingly. Please check.

AQ2 = Note that reference [57] and [109] are the same. Therefore, reference [109] was deleted from the list. Citations were renumbered accordingly. Please check.

\section{END OF ALL QUERIES}

